\title{
INTRASEASONAL DYNAMICAL EVOLUTION OF THE NORTHERN ANNULAR MODE
}

\author{
A Dissertation \\ Presented to \\ The Academic Faculty \\ By \\ Brent A. McDaniel \\ In Partial Fulfillment \\ Of the Requirements for the Degree \\ Doctor of Philosophy in Earth and Atmospheric Science \\ Georgia Institute of Technology \\ May, 2005
}




\section{INTRASEASONAL DYNAMICAL EVOLUTION OF THE NORTHERN ANNULAR MODE}

Approved by:

Dr. Robert X. Black, Chairman

Dr. Derek Cunnold

School of Earth and Atmospheric Sciences

School of Earth and Atmospheric Sciences

Georgia Institute of Technology

Georgia Institute of Technology

Dr. Robert Dickinson

Dr. Rong Fu

School of Earth and Atmospheric Sciences

Georgia Institute of Technology

School of Earth and Atmospheric Sciences

Georgia Institute of Technology

Dr. Walter Robinson

School of Atmospheric Sciences

Date Approved: April 11, 2005

University of Illinois 


\section{ACKNOWLEDGMENTS}

During ones tenure as a graduate student, various individuals stand out as deserving recognition and acknowledgment. Their contribution may come in many forms: scientific advice, computing assistance, providing data, proofreading papers. These key persons help the scientific process occur and their help was crucial in the work that went into this document. But I would be remiss if I excluded the less tangible (but no less important!) support that I have received not just during my graduate career but throughout life. Without this assistance, this document would have either never existed or been abandoned.

Specifically, I would like to thank my advisor, Robert Black. He was always there to provide guidance, answer questions, and discuss results but was also willing to let me choose my own scientific path. Sometimes these avenues were doomed from the start but he recognized that sometimes a wrong turn can lead to interesting new surroundings. Perhaps the most sincere endorsement I can offer for Rob is that if I had a change to do it all over again, knowing what I know now, I would again select him as my advisor.

For the rest of my $\mathrm{PhD}$ committee, I offer thanks for agreeing to take time out of your busy schedules to offer your guidance and expertise to make this thesis the best it could be.

I would also like to thank my friends and colleagues in the EAS department. Your scientific help and companionship have made the last several years a much more enjoyable experience. I would also like to say thanks to my officemates Dennis 
Robinson and Benton Whitesides for many fun and varied conversations, some sciencerelated, many not, that made coming to work each day a gratifying experience.

And lastly, I'd like to say thanks to my family. To my parents, who always encouraged my curiosity and dealt with my endless questions and who provided more love and support than anyone could deserve, I say thanks. To my wife Theresa, who has always been one hundred percent supportive during my long stay in graduate school, well, it's finally over. Thanks for your love, friendship, and support. 


\section{TABLE OF CONTENTS}

ACKNOWLEDGMENTS

iii

LIST OF FIGURES

vii

SUMMARY

xiv

CHAPTER 1 INTRODUCTION 1

1.1 Stratosphere-Troposphere Coupling 1

1.2 Annular Modes: History 4

$\begin{array}{ll}\text { 1.3 Annular Mode vs Teleconnection Pattern } & 7\end{array}$

1.4 Current Theoretical Frameworks 9

$\begin{array}{ll}\text { 1.5 Scientific Motivation } & 12\end{array}$

$\begin{array}{lll}\text { CHAPTER } 2 \text { METHODOLOGY } & 14\end{array}$

$\begin{array}{ll}\text { 2.1 Data } & 14\end{array}$

$\begin{array}{ll}\text { 2.2 Regression Procedure } & 17\end{array}$

2.3 Dynamical Composites 20

2.4 Transformed Eulerian Mean Diagnostics 22

2.5 Potential Vorticity Inversions 30 
3.1 General Structure 33

3.2 Lag-Regression Analyses $\quad 35$

3.3 Summary 46

CHAPTER 4 CASE STUDIES $\quad 48$

4.1 Methodology and Case Statistics $\quad 48$

4.2 Illustrative Case Studies $\quad 51$

4.3 Nonpropagating NAM Composites 62

$\begin{array}{ll}4.4 \text { Summary } & 67\end{array}$

$\begin{array}{ll}\text { CHAPTER } 5 \text { DYNAMICAL COMPOSITES } & 68\end{array}$

5.1 Methodology and Composite Statistics $\quad 68$

5.2 Positive Phase of the NAM 69

$\begin{array}{ll}\text { 5.3 Negative Phase of the NAM } & 78\end{array}$

5.4 Regional Wave Forcing and Spectral Decomposition 85

5.5 Summary 93

CHAPTER 6 SUMMARY AND CONCLUSIONS 95

$\begin{array}{ll}\text { 6.1 Summary } & 95\end{array}$

6.2 Theoretical Implications 104

$\begin{array}{ll}\text { 6.3 The Future } & 107\end{array}$

$\begin{array}{ll}\text { REFERENCES } & 109\end{array}$ 


\section{LIST OF FIGURES}

Figure 2.1 Correlations between the 90-day low-pass AO signature time series at $10 \mathrm{mb}$ in 1 January with the AO signature time series at all levels during November-March. From Baldwin and Dunkerton (1999).

Figure 3.1 Mean structure of the NAM produced by averaging all 21 lag regressions. Figure (a) shows the $1000 \mathrm{mb}$ geopotential height anomalies while figure (b) shows a latitude-height cross section for the zonally averaged zonal wind anomalies.

Figure 3.2 Polar stereographic plots for the regressed $10 \mathrm{mb}$ geopotential height anomalies for days (a) 1 - 4, (b) 5 - 8, (c) 9 - 12, (d) 13 15, (e) 16 - 18, and (f) $19-21$. See text (section 2.2) for details on the regression and mapping to a 21 day time axis. Units are geopotential meters.

Figure 3.3 Polar stereographic plots for the regressed $300 \mathrm{mb}$ geopotential height anomalies for days (a) 1 - 4, (b) 5 - 8, (c) 9 - 12, (d) 13 15, (e) 16 - 18, and (f) $19-21$. See text (section 2.2) for details on the regression and mapping to a 21 day time axis. Units are geopotential meters.

Figure 3.4 As in figure 3.4 but for the $1000 \mathrm{mb}$ geopotential height anomalies.

Figure 3.5 Zonally averaged zonal wind anomalies [m/s] for days (a) 1 - 4, (b) 5 - 8, (c) 9 - 12, (d) 13 - 15, (e) 16 - 18, and (f) $19-21$.

Figure 3.6 Anomalous EP flux vectors $\left[\mathrm{m}^{3} \mathrm{~s}^{-2}\right]$ and wave driving $[\mathrm{m} / \mathrm{s} / \mathrm{day}]$ for days (a) 9 - 12 and days (c) 16 - 18 and the Coriolis Torque $\left(\mathrm{fv}^{*}\right)[\mathrm{m} / \mathrm{s} /$ day $]$ for days (b) 9 - 12 and days (d) 16 - 18. EP flux vectors are scaled to point in the proper direction while enhancing magnitudes at successively higher altitudes (see text for details). The local EP flux divergence is not preserved but can be inferred from the wave driving.

Figure 3.7 Composite of the smoothed NAM index based on its maximum value. The solid line represents the positive phase of the NAM while the dashed line represents the negative phase times $(-1)$. 
Figure 4.1 10-day low-pass filtered NAM signature time series for 19581997. Contour values between -0.6 and 0.6 are not shaded. Red corresponds to a weak, warm polar vortex while blue corresponds to a strong, cold polar vortex. Faint vertical lines denote the November-March winter season. Red tick marks indicate major midwinter or early final warnings during DJFM. Figure provided by Mark Baldwin.

Figure 4.2 Time evolution of the zonal-mean zonal wind anomalies at $60 \mathrm{~N}$ for (upper) case 1 and (lower) case 2. Units are $\mathrm{m} / \mathrm{s}$.

Figure 4.3 Latitude-pressure cross sections of zonal mean fields during case 1 (C1) development. (a) Initial and (b) final zonal wind anomalies (UP; $\mathrm{m} / \mathrm{s})$. Nine-day changes in (c) geostrophic zonal wind $(\mathrm{UG} ; \mathrm{m} / \mathrm{s})$ and $(\mathrm{d}) \mathrm{PV}\left(1.0 \times 10^{-5} \mathrm{~s}^{-1}\right)$.

Figure 4.4 Latitude-pressure cross sections of zonal mean diagnostic fields during $\mathrm{C} 1$ development. Nine day UG changes induced by (a) stratospheric and (b) tropospheric PV anomalies $(\mathrm{m} / \mathrm{s})$. (c) Nine-day average anomalies in EP flux (vectors, $\mathrm{m}^{3} \mathrm{~s}^{-2}$ ) and associated wave driving (contours, $\mathrm{m} / \mathrm{s} / 9$ days) and (d) same as (c) but only the forcing due to the low-pass eddy flux anomalies. Scaling of the EP flux vectors same as in figure 3.6.

Figure 4.5 Latitude-pressure cross sections of zonal mean fields during case 1 (C1) development. (a) Initial and (b) final zonal wind anomalies (UP; $\mathrm{m} / \mathrm{s}$ ). (c) Initial and (d) final PV anomalies (1.0 $\left.\mathrm{x} 10^{-5} \mathrm{~s}^{-1}\right)$.

Figure 4.6 Latitude-pressure cross sections of zonal mean diagnostic fields during $\mathrm{C} 1$ development. Nine day changes in (a) total $\mathrm{UG}(\mathrm{m} / \mathrm{s})$ and (b) UG induced by stratospheric PV anomalies $(\mathrm{m} / \mathrm{s})$. (c) Nine-day average anomalies in EP flux (vectors, $\mathrm{m}^{3} \mathrm{~s}^{-2}$ ) and associated wave driving (contours, $\mathrm{m} / \mathrm{s} / 9$ days) and (d) same as (c) but only the forcing due to the low-pass eddy flux anomalies. Scaling of the EP flux vectors same as in figure 3.6. 
Figure 4.7 Latitude-pressure cross sections of zonal mean fields during case 3 (C3) development. (a) Initial and (b) final zonal wind anomalies (UP; $\mathrm{m} / \mathrm{s}$ ). Nine-day changes in (c) geostrophic zonal wind $(\mathrm{UG} ; \mathrm{m} / \mathrm{s}$ ) and (d) $\mathrm{UG}$ induced by tropospheric $\mathrm{PV}$ anomalies $(\mathrm{m} / \mathrm{s})$.

Figure 4.8 Composite analyses of the six NN annular mode events. (a) As in figure 4.2a except for the $\mathrm{NN}$ composite development. Latitude-pressure cross sections of composite zonal mean fields during $\mathrm{NN}$ development are presented in the remaining frames. (b) Initial UP (m/s). (c) Ten-day change in UG induced by stratospheric PV anomalies $(\mathrm{m} / \mathrm{s})$. (d) Ten-day average anomalies in the EP flux (vectors, $\mathrm{m}^{3} \mathrm{~s}^{-2}$ ) and associated wave driving (contours, $\mathrm{m} / \mathrm{s} / 9$ days).

Figure 4.9 As in figure 4.8 but for the composite analyses of the seven PN annular mode events.

Figure 5.1 Latitude-pressure cross sections of the zonal wind anomaly [m/s] and the zonal wind tendency $[\mathrm{m} / \mathrm{s} /$ day $]$. The green contour represents the 95\% significance level. Figures (a) and (b) show the wind anomalies and tendencies for the mature stage of the positive phase of the NAM while figures (c) and (d) show the wind anomalies and tendencies for the decline stage of the positive phase of the NAM.

Figure 5.2 Latitude-pressure cross sections for the decline stage of the positive phase of the NAM showing (a) composited zonal windtendency $[\mathrm{m} / \mathrm{s} /$ day $]$, (b) the sum of the wave driving and Coriolis torque $[\mathrm{m} / \mathrm{s} /$ day $]$, (c) the zonal wind -tendency calculated by inverting the entire domain of the PV-tendency [m/s/day], and (d) the zonal wind-tendency calculated by inverting only the stratospheric PV-tendency. [m/s/day]. 
Figure 5.3 Detailed analysis of the mature stage of the positive phase of the NAM. (a) zonal wind tendency due to stratospheric PVtendencies $[\mathrm{m} / \mathrm{s} /$ day $]$, (b) anomalous EP-flux vectors $\left[\mathrm{m}^{3} \mathrm{~s}^{-2}\right]$ and associated wave driving $[\mathrm{m} / \mathrm{s} /$ day] (only EP vectors significant at $95 \%$ are plotted), (c) meridional component of the anomalous EP-flux $\left[10^{6} \mathrm{~m}^{3} \mathrm{~s}^{-2}\right]$, (d) vertical component of the anomalous EP-flux $\left[10^{4} \mathrm{~m}^{3} \mathrm{~s}^{-2}\right]$ (e) the Coriolis torque due to the residual meridional velocity $[\mathrm{m} / \mathrm{s} / \mathrm{day}]$, and (f) the residual vertical velocity $\left[10^{-4} \mathrm{~m} / \mathrm{s}\right]$. EP flux vectors are scaled to point in the proper direction while enhancing magnitudes at successively higher altitudes (see text for details). The local EP flux divergence (which is not preserved in this scaling) can be inferred from the wave driving anomalies.

Figure 5.4 As in figure 5.3 but for the decline stage of the positive phase of the NAM.

Figure 5.5 As in figure 5.1 but for the negative phase of the NAM.

Figure 5.6 As in figure 5.3 but for the mature stage of the negative phase of the NAM.

Figure 5.7 As in figure 5.3 but for the decline stage of the negative phase of the NAM.

Figure 5.8 Three-dimensional Plumb flux anomalies for the positive (a) and (b) and negative (c) and (d) phases of the mature stage of the NAM due to the anomalous stationary waves. Figures (a) and (c) show the stationary height anomaly pattern (shaded, [units of $100 \mathrm{~m}])$ at $300 \mathrm{mb}$ and horizontal flux vectors at $300 \mathrm{mb}\left(\mathrm{m}^{2} / \mathrm{s}^{2}\right)$ while figures (b) and (d) show the horizontal flux vectors at $300 \mathrm{mb}$ and the vertical flux (contours/shading, units of 100 $\mathrm{m}^{2} / \mathrm{s}^{2}$ ) at $700 \mathrm{mb}$. 
Figure 5.9 Latitude-pressure cross sections for the mature stage of the positive NAM phase showing (a) anomalous EP-flux vectors $\left[\mathrm{m}^{3}\right.$ $\left.\mathrm{s}^{-2}\right]$ and associated wave driving [m/s/day] due to LP eddies, (b) same as (a) but the forcing due to waves $1,2,3$, (c) a corresponding analysis using the meridional and vertical components of the zonal-mean Plumb flux (derived from unfiltered composite anomalies), (d) longitude-pressure cross section of the composite geopotential height anomalies at $60 \mathrm{~N}$ ( $50 \mathrm{~m}$ contours shown).

Figure 5.10 As in figure 5.9 but for the mature stage of the negative phase of the NAM.

Figure 5.11 Climatological values of the Plumb flux (a) and (b) and the EP flux and associated wave driving (c). The climatological stationary wave pattern at $60 \mathrm{~N}$ is shown in panel (d). See figure 5.8 for details on panels (a) and (b), figure 5.3 for details on panel (c) and figure 5.9 for details on panel (d).

Figure 6.1 Polar stereographic plots showing the difference in the temperature anomaly field between the maturing and declining stages for (a) the positive phase and (b) the negative phase of the NAM. 


\section{SUMMARY}

Recent observational and modeling studies indicate a robust dynamical coupling between the stratosphere and troposphere during boreal winter. This coupling occurs in association with the Northern Annular Mode (NAM), which itself accounts for a significant fraction of the variability of the extratropical circulation. While monthly NAM dynamics have been studied previously, the mechanisms that give rise to NAM variability on short intraseasonal timescale are still unclear.

We perform regression analyses, case studies, and composites based on periods of dynamical growth/decay to investigate the roles of the different proposed mechanisms in driving the atmospheric variability observed in association with the NAM on short intraseasonal timescales. More specifically, lag-regression analyses are used to identify the mean canonical structures present during the evolution of a typical NAM event. Illustrative case studies of robust stratospheric NAM events but with different tropospheric signals are contrasted in order to identify the underlying dynamical reasons for the observed differences. Finally, composite analyses of NAM tendencies are performed to isolate the structural and dynamical evolution of NAM events. Zonal-mean and three-dimensional eddy-flux diagnoses are used to examine the role of eddy-mean flow interaction in driving the wind tendencies characteristic of the NAM. In particular, Plumb flux analyses are employed to quantify the contribution of regional stationary wave anomalies toward the zonal mean wind tendency field. Potential vorticity inversions are also used to determine the role of stratospheric anomalies in inducing tropospheric circulations. 
The case study analyses indicate that preexisting tropospheric PV anomalies can mask the downward penetration of an initial stratospheric NAM signal into the troposphere. PV inversions further suggest that a minimum requirement for a direct downward stratospheric influence is that the stratospheric NAM signal be robust in the lower stratosphere.

The dynamical composites show a remarkable degree of reverse symmetry between the zonal-mean dynamical evolution of positive and negative NAM events. Anomalous Eliassen-Palm fluxes are observed in the troposphere and stratosphere, consistent with index of refraction considerations and an indirect downward influence of the stratosphere on the troposphere. The patterns of anomalous wave driving, primarily due to low-frequency planetary scale waves, provide the main forcing of the zonal mean wind tendency field. Regional wave activity fluxes indicate that the wave driving pattern represents the manifestation of planetary scale anomalies over the North Atlantic. 


\section{CHAPTER 1}

\section{INTRODUCTION}

\subsection{Stratosphere-Troposphere Coupling}

The atmosphere is normally subdivided into regions according to the vertical temperature gradient, with temperature decreasing with height in the troposphere, then increasing with altitude in the stratosphere, followed by a decrease with height in the mesosphere, and finally increasing with height in the thermosphere. The troposphere is weakly stratified, allowing vertical displacements, while the stratosphere is strongly stratified, limiting vertical motion This contrast reflects the nature of the radiativedynamical balance in the regions. The troposphere, driven by solar heating maximized at the surface and at low latitudes, is convectively unstable in the tropics and baroclinically unstable in the extratropics. In response, large-scale motions in both the vertical and meridional plane transfer energy upward and poleward. This strong connection to the surface layer is an important characteristic of the troposphere, with significant implications for climate. (Shepherd 2002).

Above the tropopause, the temperature lapse rate changes sign as upper regions of the stratosphere are heated by the absorption of solar radiation by stratospheric ozone. This region is strongly stable, with a high static stability primarily due to the increases in ozone absorption and subsequent heating up to about $50 \mathrm{~km}$. Due to the strongly stable nature of the stratosphere, vertical motions are inhibited resulting in transport timescale that are much longer than in the troposphere. The high-latitude wintertime stratosphere is 
characterized by a cyclonic jet (encompassing a cold polar air mass) known as the polar night jet or polar vortex.

The stratosphere and troposphere have been observationally linked through different phenomenological mechanisms. These can be summarized thusly:

(i) Weather and climate: Although in weather forecasting and tropospheric climate modeling it has customarily been assumed that the role of the stratosphere is small, there is increasing observational evidence of downward influences of the stratosphere on the troposphere (e.g. Baldwin and Dunkerton 2001; Solomon 1999 ). These links may determine, for example, the effect of stratospheric aerosol changes due to volcanic eruptions on surface weather and climate and may imply a fundamental role for the stratosphere in determining future changes in the tropospheric climate associated with increases in carbon dioxide and other greenhouse gases.

(ii) Solar variability: Vertical dynamical coupling between the stratosphere and troposphere may provide an explanation for apparent signals of solar variability in tropospheric weather and climate

The stratosphere has the potential to significantly affect conditions near the Earth's surface directly in the following ways (Haynes 2005):

(a) Stratospheric ozone: Changes in stratospheric ozone imply changes in surface ultraviolet irradiance and also an altered supply of ozone to the troposphere at high latitudes. The latter may have a significant effect on surface ozone contributions (Zeng and Pyle 2003).

(b) Radiative balance of the troposphere: The lower stratosphere plays an important role in the radiative balance of the troposphere, and changes in greenhouse gases such as 
ozone and water vapor in the lower stratosphere can directly impact surface temperatures. (c) Potential vorticity induction: Large-scale PV structures in the stratosphere can induce deep circulation patterns that extend into the troposphere down to the surface (Hartley et al 1998).

Also of interest are the ways that the stratosphere may indirectly affect tropospheric weather and climate patterns. One proposed mechanism of relevance involves the alteration of the propagating characteristics of tropospheric planetary-scale waves. Specifically, changes in the vertical wind shear near the tropopause alter the index of refraction which in turn alters the meridional and vertical propagation of tropospheric Rossby waves (Chen and Robinson 1992, Hartmann et al. 2000). Changes in the wind shear can deflect upwardly propagating Rossby waves equatorward with less wave activity reaching the stratospheric polar vortex. The redirection of wave activity within the troposphere provides anomalous wave driving signatures within the middle and upper troposphere. Thus this mechanism provides an indirect stratospheric influence in which tropospheric waves act to alter the tropospheric zonal wind field. Further, the reduction of wave breaking in the polar vortex results in an anomalously strong polar jet and can feedback on vertical wind shear, ducting more tropospheric wave activity away from the polar vortex. This indirect mechanism may affect the forcing and propagation characteristics of both synoptic (Polvani and Kushner 2002, Song and Robinson 2004) and planetary scale tropospheric waves (DeWeaver and Nigam 2000, Hartmann et al. 2000). 


\subsection{Annular Modes: History}

Zonally symmetric or annular modes of climate variability have been an area of interest for atmospheric scientists since at least the 1930s, when C. G. Rossby and collaborators suggested that the position of the wintertime centers of action are dependent on the strength of the zonally symmetric circulation (Rossby 1939). Rossby noted that the strength of the midlatitude zonal wind was highly correlated with the position of the Aleutian low during the winter of 1938-1939. Because changes in location of the Aleutian low lagged variations in the zonal wind field at $45 \mathrm{~N}$, Rossby posited that the strength of the zonal circulation influences the position of longitudinally varying centers of action.

Willett (1948) and Rossby and Willett (1948) attributed the variability in the longitudinal location of the semi-permanent centers of action to a new paradigm, whereby low frequency climate variability of the northern hemisphere circulation is linked to fluctuations between two discrete climate states based on the strength of the zonal circulation at $45 \mathrm{~N}$. The two states, denoted as high and low index states, partially describe the wintertime mean flow. The low (high) index state is characterized by a weak (strong) midlatitude zonal flow, increased mid- (high-) latitude baroclinicity, and a geographically extended (contracted) polar vortex.

Lorenz (1951) and Namias (1950) also explored the variability in the zonally symmetric zonal flow. Independently, both found that the largest correlation between the 
strength of the zonal circulation and the location of various centers of action was at $55 \mathrm{~N}$, not $45 \mathrm{~N}$ as found by Rossby and Willett. Namias ascribed the variability as due to a cycle of meridional shifts in the location of the maximum zonal flow between $35 \mathrm{~N}$ and $55 \mathrm{~N}$, calling it the "zonal index cycle". Namias theorized that the cyclical nature of the zonal index would be useful in long-range weather forecasting. However, the temporal cycles and weather patterns theorized by Namias failed to arise in observational data and the zonal index paradigm lost favor.

As the interest in the zonal index cycle waned, researchers began focusing on teleconnection patterns in the tropospheric geopotential field (e.g. van Loon and Rogers 1978, Hoskins and Karoly 1981). Unlike the zonal-index cycle, these patterns were presumed to owe their existence to zonally asymmetric sources of heat and/or momentum (Thompson et al. 2002). One of the teleconnection patterns studied was the North Atlantic oscillation (NAO). The NAO is usually characterized as a north-south dipole anomaly in the sea level pressure (SLP) field over the North Atlantic (Walker and Bliss 1932, Hurrell 1995). The NAO can be represented by an index derived as the difference in the normalized SLP anomaly field between Lisbon, Portugal and Stykkisholmur, Iceland (Hurrell 1995). The NAO is often (but not always) linked to changes in sea surface temperatures (SSTs) (e.g. Hastenrath and Greischar 2001) with some considering the NAO to be a coupled atmosphere-ocean mode.

More recently, scientists have begun to concentrate on hemispheric modes of variability in the SLP (and other) fields. Thompson and Wallace $(1998,2000)$ found that by performing an empirical orthogonal function (EOF) analysis on the wintertime SLP anomaly field, robust zonally symmetric patterns explaining $22 \%$ of the total variance 
north of $20 \mathrm{~N}$ emerged. They christened this new pattern the "Arctic Oscillation" (AO). Baldwin and Dunkerton (1999) examined the geopotential height anomaly field and found results remarkably similar to Thompson and Wallace's AO structures. The AO pattern is similar to the NAO pattern, with negative height anomalies over the pole and positive anomalies over the North Atlantic. Both account for a large fraction of the extratropical wintertime tropospheric variability in the northern hemisphere circulation. Unlike the NAO pattern, however, positive anomalies (with similar magnitudes to those seen over the North Atlantic) are also present over the Pacific basin in association with the AO. The presence of both an Atlantic and Pacific center of action led to the terminology "northern annular mode" (NAM). The current trend in the literature is to use the term $\mathrm{AO}$ to refer to the near surface manifestation of northern annular mode while the NAM refers to the entire 3-D structure of the annular mode. For the remainder of the thesis, we will use the term NAM to represent the northern annular mode at all levels. Indices representing the $\mathrm{NAO}$ and $\mathrm{AO}(\mathrm{NAM})$ are as highly correlated as $92 \%$ (Ambaum et al. 2001). ${ }^{1}$

\subsection{Annular Mode vs Teleconnection Pattern}

The NAM perspective suggests that the NAO is not purely a regional teleconnection pattern but contains a zonally symmetric pattern reminiscent of the structure examined by Rossby, Willett, Lorenz, and Namias (Thompson et al 2002). The

\footnotetext{
${ }^{1}$ Regardless of the exact indices used to represent the $\mathrm{NAO}$ and $\mathrm{AO}$, the indices remain highly correlated.
} 
principal arguments in favor of an annular mode paradigm over the historical regional NAO perspective, where the variability is associated only with dynamics due to the North Atlantic stormtrack, are (Thompson et al. 2002):

(i) The zonal mean structure of the NAM is virtually identical to that of the widely recognized Southern Hemisphere annular mode (SAM) (e.g. Limpasuvan and Hartmann 1999).

(ii) The NAM structure is evident in the first EOF of the height and zonal wind fields at levels throughout the troposphere and stratosphere (Baldwin and Dunkerton 1999, Thompson and Wallace 2000).

(iii) Both the NAO and NAM patterns capture recent trends in geopotential heights that have occurred over the last decades. The hemispheric scale trends are better recovered, however, by regressions upon the NAM index compared to the NAO index (Thompson and Wallace 1998). If the variability were confined to the North Atlantic, one would not expect the decadal variability to have such large amplitudes throughout the hemisphere. (iv) The projection of the first principal component of stratospheric height anomalies onto the surface geopotential height field has a center of action over the Pacific, albeit a weaker one than that over the North Atlantic (Baldwin and Dunkerton 1999).

(v) Variability in the occurrence of blocking episodes and cold air outbreaks over the Atlantic and Pacific are accounted for by the NAM index.

An important question is, why does the choice of perspective matter? Wallace (2000) detailed the similarities and distinctions between the two paradigms. In the 
regional (NAO) perspective, the dynamics are unique to the North Atlantic stormtrack. In this case, the dynamics are representative of a coupled ocean-atmosphere system operating within/over the North Atlantic sector (Grotzner et al. 1998, Rodwell et al. 1999).

Both perspectives rely on local eddy-mean flow interactions to drive the variability observed in the NAO/NAM. However, the NAO relies on storm track variability coupled to local circulations. The annular mode perspective suggests that hemispheric scale circulations work to organize local eddy activity, potentially through eddy-mean flow interactions and feedbacks (Lorenz and Hartmann 2001,2003).

The choice between the different paradigms also has implications for the coupling between the troposphere and stratosphere. The NAO framework suggests that the observed coupling between the stratosphere and troposphere is via a distinctive wavelike pattern in the middle troposphere over the North Atlantic. In the NAM framework, the stratosphere-troposphere coupling is intrinsically linked to the dynamics of the polar vortex itself. Stratospheric processes (that may or may not have their roots in tropospheric forcings) can alter the strength and size of the stratospheric polar vortex, nudging the extratropical atmosphere out of thermal wind balance. These changes can induce vertical secondary circulation that acts to restore the thermal wind balance (e.g. Haynes et al. 1991). The resulting non-local changes can extend downward into the troposphere to the Earth's surface (Black 2002). As mentioned earlier, changes in the stratospheric polar vortex can also affect the propagation of tropospheric waves, indirectly altering the tropospheric circulation.

The choice of perspective has led to much debate and generated a body of 
literature. The answer as to which perspective is "correct" is largely unanswered and perhaps unanswerable. Regardless, the results presented in this thesis are not dependent on a particular paradigm although their interpretation may.

\subsection{Current Theoretical Frameworks}

No individual theory completely explains the interaction between the stratosphere and troposphere during wintertime NAM events. Most of the current theories involve some combination of the following dynamical processes:

(a) Vertical reflection of upwardly propagating Rossby waves [originating within the troposphere] within the stratosphere (Perlwitz and Harnik 2003).

(b) Direct forcing of the tropospheric circulation by stratospheric potential vorticity (PV) anomalies. Nonlocal geostrophic and hydrostatic adjustments occur in association with anomalies in the lower stratospheric PV field (Hartley et al. 1998). Specifically, potential vorticity anomalies associated with variations in the strength of the stratospheric polar vortex induce circulation anomalies in the troposphere (Ambaum and Hoskins 2002, Black 2002, Black and McDaniel 2004, McDaniel and Black 2005).

(c) Variations in the lower stratospheric zonal wind alter the upward propagation of tropospheric Rossby waves. In this mechanism, changes in the near-tropopause stratospheric winds alter the vertical wind shear. Changes in the vertical wind shear can duct tropospheric wave activity equatorward (poleward), leading to anomalous wave driving in the middle and upper troposphere. Enhanced equatorward (poleward) wave activity results in less (more) wave activity propagating into the stratosphere. This 
secondary feedback leads to an enhancement (slowing) of the stratospheric polar vortex (e.g. Hartmann et al. 2000, Limpasuvan et al. 2004, McDaniel and Black 2005, Lorenz and Hartmann 2003)). The wave driving due to the tropospheric Rossby waves are associated with latitudinal PV fluxes in both the troposphere and stratosphere, potentially linking mechanisms $\mathrm{C}$ and $\mathrm{B}$.

Even a concept as fundamental as the initiation mechanism for NAM events still remains unresolved. The essential question is in what region do NAM events first arise, a question that thus far remains unclear. NAM events exhibit remarkable variability in the manner in which they originate: some appear first in the troposphere with a subsequent signal following in the stratosphere; others arise in the stratosphere and then emerge in the troposphere; while others materialize in the troposphere (stratosphere) with no subsequent signal appearing in the stratosphere (troposphere). By examining tropospheric eddy heat fluxes near the tropopause, Polvani and Waugh (2004) found that extreme stratospheric events are nearly always preceded by anomalous upward wave activity. From this they inferred that most extreme stratospheric events originate in the troposphere.

While most theories include a wave-mean flow interaction component, the relative role of synoptic and low frequency waves is still unclear. DeWeaver and Nigam (2000a, 2000b) found that the momentum fluxes due to interactions between climatological and anomalous stationary waves constituted the primary source of momentum forcing during monthly mean NAM events. They also found that the zonal wind anomalies and stationary waves are well coupled, providing a strong positive feedback between the zonal mean and eddy flow components. Limpasuvan et al. (2004), 
studying sudden stratospheric warmings (SSWs), recently found that, in contrast to DeWeaver and Nigam, synoptic scale waves (wavenumbers 4 and higher) in the upper troposphere are responsible for the majority of the anomalous momentum fluxes. One should note however, that while SSWs share many of the overall characteristics with the negative phase of the NAM, the precise correspondence between the two is still in question. This topic will be further addressed in later chapters. With the exception of Limpasuvan et al. (2004), most previous diagnostic studies of the NAM have focused on monthly mean dynamical balances. Understanding the daily tendency dynamics is crucial for understanding the short-timescale behavior of the NAM.

One issue with current theories is that the direct downward influence mechanisms postulated above are generally considered to be insufficient to explain the observed tropospheric manifestation of the NAM. Various feedback mechanisms have been put forth (e.g. Robinson 2000; Wittman et al. 2004, Song and Robinson 2004) in which downward stratospheric influences are subsequently amplified in the troposphere but consensus among the scientific community has thus far not been reached.

\subsection{Scientific Motivation}

Annular modes account for a large fraction of the observed extratropical atmospheric variability in the wintertime northern hemisphere circulation in both the troposphere and stratosphere (e.g. Baldwin and Dunkerton 1999, Thompson and Wallace 1998). While tropospheric weather patterns tend to change on time scales of a few days, circulation regimes in the stratosphere tend to persist for several weeks or more. The 
long-held view is that the stratosphere only weakly impacts the lower atmosphere, thus providing little additional information for numerical weather forecasts However, recent case studies (e.g. Black and McDaniel 2004) have shown that large stratospheric circulation anomalies can extend into the troposphere and directly influence surface weather patterns.

By studying daily surface pressure maps for periods following robust stratospheric annular mode events, Baldwin and Dunkerton (2001) found that during the 60 days post-event the surface pressure pattern closely resembles the NAM pattern. They also found these large stratospheric events preceded shifts in the probability distribution of extreme NAM surface events. These surface events are statistically linked to cold air outbreaks and extreme weather conditions across Europe, Asia, and North America (Thompson and Wallace 2001). The observed changes in surface weather regimes are important for medium range weather forecasting and may be anticipated by identifying robust stratospheric events, thus adding medium to extended range predictive skill for extreme weather events.

Variations in the NAM are also linked to modulations in the Atlantic and Pacific storm tracks (Baldwin and Dunkerton 2001). During the positive (negative) phase of the NAM, both the Atlantic and Pacific storm tracks are displaced northward (southward). Regional climate trends have also been linked to the state of the NAM. During the positive phase, Alaska and western Canada experience a warm, wet winter while the Mediterranean (northern Europe) region is characterized by decreased (increased) rainfall. In the negative phase, western North America and Northern Europe experience a cold winter while the Mediterranean is unusually stormy. 
The ability to predict day-to-day variability in the NAM will likely provide improved short-term weather forecasts of extreme events. Predicting intraseasonal changes in the NAM (changes in NAM phases or regimes) can improve long-term forecasts in much the same way that El-Nino/Southern Oscillation predictability improves midlatitude long-range forecasts. This improved forecast ability would enable the energy, food production, and other industries to better prepare for coming weeks. Thus an improved dynamical understanding of the mechanisms responsible for short term variations in the NAM has important scientific and practical implications. 


\section{CHAPTER 2}

\section{METHODOLOGY}

\subsection{Data}

The primary source of data used in this study is the National Center for Environmental Prediction/National Center for Atmospheric Research (NCEP/NCAR) reanalysis dataset (Kalnay et al. 1996). The reanalysis combines a comprehensive collection of observed fields including marine and land surface observations, satellite data, radiosondes, aircraft data, and other observed fields using a global, temporally fixed assimilation system to produce a self-consistent gridded dataset archived on a $2.5^{\circ} \times 2.5^{\circ}$ horizontal grid with 17 pressure levels spanning from the surface to the middle stratosphere $(1000 \mathrm{mb}$ to $10 \mathrm{mb})$. One advantage of using a time invariant assimilation system is to removed perceived climate shifts associated with the analysis system noting that erroneous trends associated with evolving observational input may remain. This study focuses on daily averaged data [zonal, meridional, and vertical winds as well as temperature and geopotential heights] derived from the 4-times daily reanalysis for the 40 year time period 1958 to 1997.

A long-term seasonal trend for each field was constructed by calculating the daily average over the 40 year period of study and then smoothing the resulting annual cycle by applying a 91-point Parzen filter (Press et al. 1992). The goal of applying the filter was to generate a smooth seasonal trend that would represent the effective climatology sampled from considerably more than 40 years of data. The resulting annual trend is hereafter 
referred to as the climatological mean. Anomaly fields are then defined as the deviations from these long-term trends and will be denoted with an asterisk (i.e., $u^{*}$ ).

To investigate the role of eddies on different timescales, the anomaly fields were decomposed into distinct frequency bins using a 151-point Lanczos filter (Duchon, 1979). Bandpass (BP) eddies are defined as having periods of 2-10 days (synoptic timescales), lowpass (LP) eddies are those having periods of 10 days - 1 year (intraseasonal variability that includes atmospheric blocking and teleconnection patterns), and interannual eddies are those having timescales greater than 1 year. It is worth noting up front that eddies occurring on interannual timescales contribute little to the dynamics presented in this thesis. The Lanczos filter, despite being more computationally intensive than some other less sophisticated filters, was used due to the minimal ringing in its response function, meaning minimal signal is allowed to pass outside the desired frequency band.

To further investigate the role of different eddy forcings, the input data were also spatially decomposed into respective zonal wavenumbers using fast Fourier transform filters (Press et al. 1992). Specifically, for each day we partition the eddy field into planetary scale waves (wavenumbers 1,2, and 3; spatial scales usually linked to LP time scales) and shorter waves (wavenumber 4 and higher; usually linked to synoptic timescales).

To characterize the northern annular mode, the multi-level NAM index of Baldwin and Dunkerton (Baldwin and Dunkerton 1999; hereafter BD99) was used. This index was calculated as follows:

The daily geopotential fields north of $20 \mathrm{~N}$ at 1000,300,100,30, and $10 \mathrm{mb}$ were first normalized by the standard deviation at each level. These 5 levels represent a 
sampling of the atmosphere from the surface to the stratosphere and were treated as a single field. A 90-day low-pass filter was then applied to this 4-D dataset. To insure that higher latitudes were not overly emphasized, the data was resampled onto an equal area grid and an empirical orthogonal function (EOF) analysis was performed on the December-January-February (DJF) subset of this data. The Arctic Oscillation (AO) pattern was identified as the leading principal component (the one explaining the most variance) of the temporal covariance matrix of the DJF subset. It is worth noting that the AO pattern calculated in BD99 by regressing the $1000 \mathrm{mb}$ geopotential field onto their AO index (calculating by using data spanning the troposphere into the stratosphere) is nearly identical to the sea-level pressure (SLP) AO pattern calculated by Thompson and Wallace (1998), calculated using only data at $1000 \mathrm{mb}$.

The analysis of BD resulted in a single daily AO index with 14906 points (40yrs of daily data). To examine lag relations of the NAM at various altitudes, the AO index needed to be mapped onto the 17 separate pressure levels in the dataset. Regressing the $\mathrm{AO}$ index for each level yields an $\mathrm{AO}$ signature, a projection of the geopotential at a given pressure level onto the $\mathrm{AO}$ pattern. Adopting the notation used in $\mathrm{BD} 99$, if $Z 10_{\mathrm{AO}}$ is the regressed $10 \mathrm{mb}$ signature of the $\mathrm{AO}$ (time invariant) and $Z 10$ is the daily map of the $10 \mathrm{mb}$ geopotential then:

$$
\min \left|Z 10-\alpha Z 10_{A O}\right|^{2}
$$

defines $\alpha$ as the regression coefficient (multiple of $Z 10_{\mathrm{AO}}$ ) that results in the "best fit" of the AO signature to the daily map. This calculation was performed separately for each day at all 17 pressure levels, resulting in a 17-level, 14906 point index, hereafter referred 
to as the (multi-level) NAM index. By convention, positive values of the NAM index correspond to an anomalously strong, cold polar vortex while negative values represent an anomalously weak, warm polar vortex.

\subsection{Regression Procedure}

A commonly used statistical tool is a regression analysis in which a field is regressed onto a previously calculated index. This operation extracts the portion of the field that projects onto the phenomenon described by the index. As an example, regressing sea surface temperatures onto an El Nino index will result in the familiar eastwest temperature dipole in the tropical pacific. The mathematical form of the regression operation is given by:

$$
A_{N A M}=\frac{1}{m} \sum_{n} a(n) I(n)
$$

where $A_{N A M}$ is the regressed value of an observed, $a$ is the time-dependent field being regressed, $I$ is the index representing the pattern (in this case the NAM index), both of which have $m$ total points. Such regression operations are quite straightforward and commonplace in the atmospheric sciences.

BD99 found that if a lag-correlation technique was applied to the multi-level NAM index, a pattern of downward propagation appeared. Figure 2.1 (figure 5 from BD99) shows this propagation pattern as a line of maximum correlation that slowly descends from the stratosphere to the troposphere, taking approximately 3 weeks. This figure was 


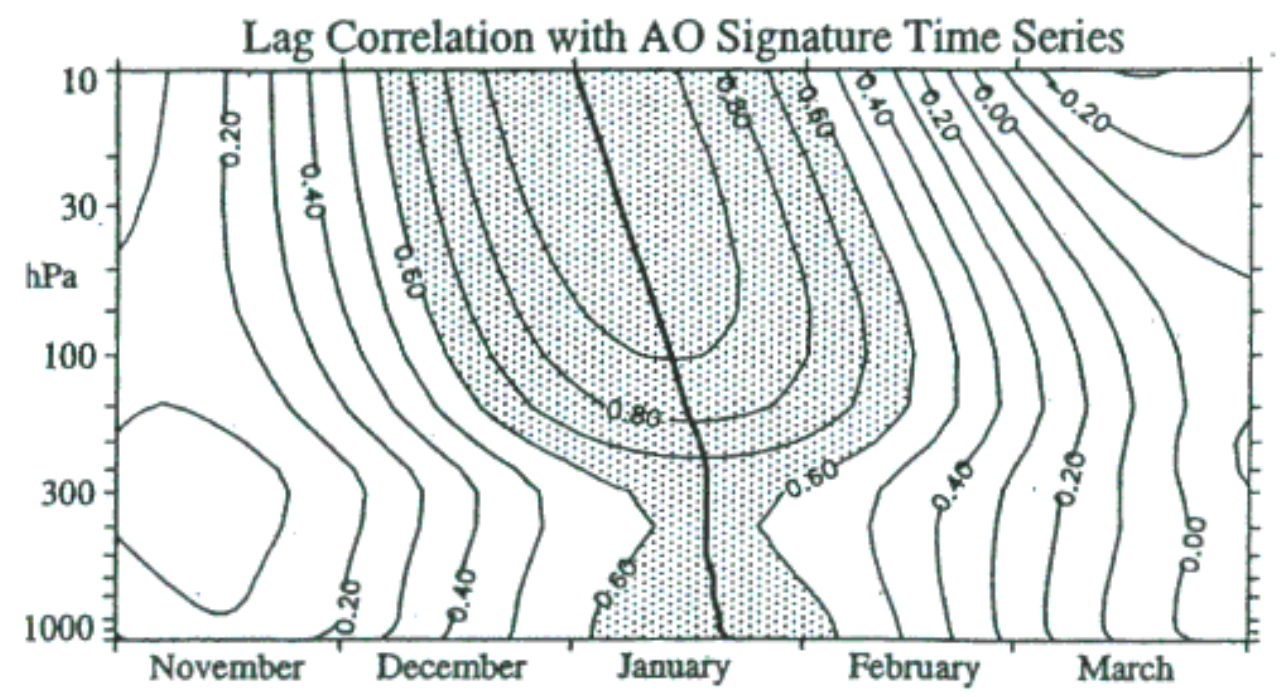

Figure 2.1 Correlations between the 90-day low-pass AO signature time series at $10 \mathrm{mb}$ in 1 January with the AO signature time series at all levels during November-March. From Baldwin and Dunkerton (1999). 
produced by correlating the $10 \mathrm{mb}$ NAM index on 1 January with the NAM index at all other levels from November through March. Examining the downward propagation carefully, we see that the propagation accelerates (slope of the maximum correlation line increases) once the signal reaches the troposphere. Using the paradigm of downward propagation of the NAM signal, we can apply lag regressions to further investigate the short time-scale dynamics of the NAM descent.

The details of the lag regressions are as follows:

First a three-dimensional field (e.g. zonal wind) is regressed onto the index for each level, creating 17 (1 per pressure level) regressed fields. Each of these regressed fields is denoted by a lag (based on the level of the NAM index used in that particular regression) that corresponds to a particular stage in the NAM evolution. For example, the three-dimensional field that results from regressing a field onto the $10 \mathrm{mb}$ NAM index is lag 0 , the regression that uses the $20 \mathrm{mb}$ NAM index would be lag 1 , and so on with lag 16 denoting the regression result obtained by using the $1000 \mathrm{mb}$ NAM index. This procedure results in, for a given field, 17 three-dimensional regression patterns. These 17 patterns are then mapped into the time domain by using a cubic-spline interpolation (Press et al 1992) onto a 21-day time axis determined from figure 1. The mapping takes into account the change in rate of descent that occurs as the signal progresses from the stratosphere into the troposphere. This procedure results in a three-dimensional evolution that shows the NAM signal descending from the stratosphere to the surface in the zonal, meridional, and vertical wind anomaly fields as well as geopotential height and temperature anomaly fields. 


\subsection{Dynamical Composites}

Although the regression analyses are well suited to identify typical structures, they were less effective at isolating tendencies in the NAM. To better understand the intraseasonal tendency dynamics associated with the northern annular mode, composites were performed based on the slope of the Baldwin-Dunkerton NAM index with the goal of identifying periods of intensification/weakening in the NAM. The goal of the “dynamical composites" being to identify the dynamical forcings leading to NAM tendencies. A further advantage of the composite analyses is that they allow a separate assessment of the opposite phases of the NAM

To identify periods of dynamical interest within the NAM, we identify two index time series - one is the NAM index itself; the second is the time rate of change of the NAM index, hereafter referred to as the index slope. The standard deviation of the NAM index is denoted as $\sigma$ while the standard deviation of the index slope is denoted by $\sigma_{s}$.

The Baldwin-Dunkerton NAM index was detrended and normalized ( 0 mean, unit standard deviation) for the winter months (DJF). The index was also lightly smoothed with a 10-day running mean operator to remove some noise. The standard deviation of the index slope, $\sigma_{s}$, was calculated based solely on DJF months for each vertical level. The composites are based on both of these time series as follows:

The procedure first identifies periods during which the time rate of change of the NAM index (index slope) at a specific vertical level is greater (less) than two standard 
deviations, $2 \sigma_{s}\left(-2 \sigma_{s}\right)$, per 12 hour time interval. [The same analyses were also

performed for $1 \sigma_{s}$ providing results that were qualitatively and quantitatively similar but

somewhat more noisy.] These cases are then classified based on the initial sign and magnitude of the NAM index. The bins used are: onset (slope $>2 \sigma_{\mathrm{s}}$, index is $0<.5$ ), growth (slope $>2 \sigma_{\mathrm{s}}$, index $.5<1$ ), maturing (slope $>2 \sigma_{\mathrm{s}}$, index $>1$ ), declining (slope $<-$ $2 \sigma_{\mathrm{s}}$, index $>1$ ), decay (slope $<-2 \sigma_{\mathrm{s}}$, index $.5<1$ ), and termination (slope $<-2 \sigma_{\mathrm{s}}$, index $0<$ .5). Index values greater (less) than $1(-1)$ represent robust positive (negative) NAM events. The same procedure is also used to identify negative phase cases with oppositely signed derivatives and indices. The individually categorized cases are then composited together, resulting in a three-dimensional depiction of the atmosphere during times when the index is changing within a respective NAM phase. This approach leads to 12 individual composites based on the index at each vertical levels. The composite operation is applied separately to the individual terms in eqt. 2.10 to study NAM tendency dynamics. The results for the $150 \mathrm{mb}$ index are presented in this thesis since it provides synthesized information on the joint tropospheric and stratospheric evolution.

\subsection{Transformed Eulerian Mean Diagnostics}

A useful diagnostic paradigm for examining large-scale extratropical dynamics in the free troposphere and stratosphere is the quasi-geostrophic (QG) framework. Under the QG framework, vertical motions are, to first order, zero while the horizontal winds are 
assumed to be in geostrophic balance.

A useful coordinate system transformation is to replace the standard height coordinate with a log-pressure vertical coordinate given by

$$
z^{*} \equiv-H \ln \left(p / p_{s}\right)
$$

where $\mathrm{H}$ is a standard scale height ( $7 \mathrm{~km}$ is used in this thesis) and $p_{s}$ is a standard reference pressure $(1000 \mathrm{mb}$ is used here). For convenience throughout the remainder of the thesis, the asterisk notation used to distinguish the log-pressure vertical coordinate (from geometric height) will be dropped with the understanding that $z$ will denote logpressure height. The vertical wind is defined as the time derivative of the log-pressure vertical coordinate.

Using QG approximations and log-pressure coordinates, the zonal momentum equation can be written as

$$
\frac{D u}{D t}-f v+\frac{\partial \Phi}{\partial x}=X
$$

where $X$ is frictional drag due to small-scale processes and the material derivative is given by

$$
D / D t \equiv \partial / \partial t+u \partial / \partial x+v \partial / \partial y+w \partial / \partial z
$$

which determines the time rate of change of a quantity following the material. The thermodynamic energy equation can be expressed as 


$$
\frac{D T}{D t}+\left(\frac{\kappa T}{H}\right) w=\frac{J}{c_{p}}
$$

with $\kappa \equiv R / c_{p}$ representing the ratio of the gas constant to specific heat $\left(c_{p}\right)$ and $J$

representing the diabatic heating rate. If the zonal flow is partitioned into its zonal mean (denoted with an overbar) and the departure from the zonal mean (denoted with a prime, representing the waves or eddies), we can rewrite eqt 2.4 as

$$
\overline{\partial u} / \partial t=\bar{f} \bar{v}-\frac{\partial}{\partial y}\left(\overline{u^{\prime} v^{\prime}}\right)+D_{x}
$$

which is the standard Eulerian mean zonal momentum equation (ignoring terms considered small in a QG scale analysis). A quantitative examination of the individual terms in eqt 2.7 (e.g., O'neill and Taylor 1979) reveals that the zonal mean acceleration is often a small residual between the Coriolis force and the eddy momentum divergence. In the absence of wave dissipation or transience, the waves act to establish an Eulerian mean meridional circulation that largely acts to balance the effects of eddy heat and momentum forcing. The transformed Eulerian mean formulation can be thought of as the residual dynamical balance upon removing this balanced circulation (Palmer 1981).

The Eulerian mean equations have a disadvantage in that it is difficult to directly diagnose the net eddy forcing on the mean flow. The transformed Eulerian mean (TEM) equations allow for a more direct attribution of the role of eddies in accelerating the mean flow. It is useful to adopt a spherical coordinate system for the remainder of the 
discussion for easier comparison with existing literature. The TEM equations are obtained by defining a residual circulation $\left(\overline{v_{r}}, \overline{w_{r}}\right)$ given by

$$
\overline{v_{r}} \equiv \bar{v}-e^{z / H} \frac{\partial}{\partial z}\left(e^{-z / H} \frac{\overline{v^{\prime} \theta^{\prime}}}{\overline{\theta_{z}}}\right)
$$

and

$$
\overline{w_{r}} \equiv \bar{w}+\frac{1}{r \cos \phi} \frac{\partial}{\partial \phi}\left(\cos \phi \frac{\overline{v^{\prime} \theta^{\prime}}}{\bar{\theta}_{z}}\right)
$$

Using the residual velocities we can manipulate eqt 2.7 into a new zonal mean momentum equation given by

$$
\frac{\partial \bar{u}}{\partial t}=f \overline{v_{r}}+\frac{e^{z / H}}{r \cos \phi} \underset{\sim}{\nabla} \cdot \underset{\sim}{F}+D_{x}
$$

where $D_{x}$ is frictional drag (negligible in the free atmosphere), $r$ is the Earth's radius, and $\underset{\sim}{F}$ is the Eliassen-Palm (EP) flux (Palmer 1981). The EP flux is expressed as

$$
\underset{\sim}{F}=r \cos \phi e^{-z / H}\left(-\overline{u^{\prime} v^{\prime}}, f \frac{\overline{v^{\prime} \theta^{\prime}}}{\overline{\theta_{z}}}\right)
$$

Eliassen-Palm fluxes provide a direct measure of Rossby-wave propagation in the meridional plane (Edmon et al 1980). The divergence of the EP flux, seen in eqt 2.10, is directly related to both (i) the meridional flux of potential vorticity and (ii) the net eddy- 
forcing (wave driving) of the zonal mean wind (Andrews et al 1987). The balance shown in eqt 2.10 can be thought of as an interplay between the Coriolis torque (first term on RHS) and the net mechanical wave driving (second term on RHS). The wave driving term explicitly quantifies the role of the eddies in modifying the zonal flow, a fundamental question in this research. Nonzero wave driving locally accelerates/decelerates the zonal wind field, leading to an induced residual secondary circulation that acts to restore the atmosphere to thermal wind balance.

It is easier to isolate the influence of large-scale dynamical forcings by examining the role of the anomalies in the context of equations $2.8-2.11$. This seemingly trivial extension of these equations is perhaps more subtle than what is seen at first glance. Because the eddy fluxes are non-linear, special care must be exercised in determining exactly which flux is the proper 'anomaly': Should the flux of the anomalies be used? The anomalous flux? Or some other combination? The answer can be ascertained by expanding the above equations into their climatological and anomaly fields as detailed below:

Any field can be decomposed into zonal and long-term means as well as the deviations from those means. Take for example a field A:

$$
A=\bar{A}_{c}+\overline{A^{*}}+A_{c}^{\prime}+A^{* *}
$$

where the subscript $c$ represents the climatological values and stars denote deviations from the long-term mean. Because the input to the EP flux requires only those fields with the zonal mean removed, we can write the input flux as 


$$
u^{\prime} v^{\prime}=\left(u_{c}^{\prime}+u^{* *}\right) \cdot\left(v_{c}^{\prime}+v^{* *}\right)=u_{c}^{\prime} v_{c}^{\prime}+u_{c}^{\prime} v^{\prime *}+u^{* *} v_{c}^{\prime}+u^{* *} v^{\prime *}
$$

In a physical sense, the RHS of equation 2.13 is the sum of the stationary eddy flux (first term), the transient-stationary eddy interaction (second and third term) and the transient eddy flux (fourth term).

To demonstrate we apply the eddy decomposition shown in eqt 2.13 to the budget described in eqt $2.7^{1}$ and expanding terms into their climatological and anomaly values yields

$$
\frac{\partial\left(\overline{u_{c}}+\overline{u^{*}}\right)}{\partial t}=f\left(\overline{v_{c}}+\overline{v^{*}}\right)-\frac{\partial}{\partial y}\left(\overline{u_{c}^{\prime} v_{c}^{\prime}+u_{c}^{\prime} v^{\prime *}+u^{*} v_{c}^{\prime}+u^{*} v^{*}}\right)
$$

Taking a climatological average of equation 2.14 yields

$$
\frac{\partial \overline{u_{c}}}{\partial t}=f \overline{v_{c}}-\frac{\partial}{\partial y}\left(\overline{u_{c}^{\prime} v_{c}^{\prime}+\left(u^{\prime *} v^{\prime *}\right)_{c}}\right)
$$

Subtracting 2.15 from 2.14 yields

$$
\frac{\partial \overline{u^{*}}}{\partial t}=f \overline{v^{*}}-\frac{\partial}{\partial y}\left(\overline{u^{* *} v_{c}^{\prime}+u_{c}^{\prime} v^{* *}+u^{* *} v^{*}-\left(u^{* *} v^{* *}\right)_{c}}\right)
$$

${ }^{1}$ An analagous expansion can be performed on eqt 2.10 yielding similar results but the exercise is more cumbersome due to the additional terms contained in the divergence of the EP flux. 
Egt 2.16 gives the budget equation for transient zonal wind anomalies, showing that the input eddy fields are a combination of the anomalous transient fluxes (the difference shown in the last 2 terms on the RHS) as well as the transient eddy-climatological stationary wave interaction (previous 2 terms). The anomalous time-tendency TEM budget analogous to eqt 2.16 will be used frequently to analyze the short-term variability crucial to understanding the intraseasonal NAM dynamics. A similar derivation can be performed to examine the partitioning of the transient eddy flux into separate time frequency bands (not shown). In this case we would again see an interaction between the climatological stationary waves and the eddies (here the eddies would be associated with specific frequency components) as well as interactions between the various frequency bands. While these interactions among frequency bands (e.g. bandpass and lowpass) cannot be ruled out a priori, in practice they were found to provide little contribution.

To assess potential regional sources and sinks of large-scale quasi-stationary Rossby wave activity, the 3-dimensional wave activity flux (Plumb 1985, hereafter referred to as the Plumb flux) is employed, expressed as:

$$
{\underset{\sim}{s}}_{F}=p \cos \phi\left[\begin{array}{l}
v^{\prime}-\frac{1}{2 \Omega \sin 2 \phi} \frac{\partial\left(v^{\prime} \Phi^{\prime}\right)}{\partial \lambda} \\
-u^{\prime} v^{\prime}+\frac{1}{2 \Omega \sin 2 \phi} \frac{\partial\left(u^{\prime} \Phi^{\prime}\right)}{\partial \lambda} \\
\frac{2 \Omega \sin \phi}{S}\left[v^{\prime} T^{\prime}-\frac{1}{2 \Omega \sin 2 \phi} \frac{\partial\left(T^{\prime} \Phi^{\prime}\right)}{\partial \lambda}\right]
\end{array}\right]
$$


is a static stability measure (the caret indicating an areal average over the region north of $20 \mathrm{~N}), \Phi$ is geopotential, and $\lambda$ is longitude. The Plumb flux provides direct information on 3-D Rossby wave activity propagation as the flux is parallel to the group velocity of quasi-stationary Rossby waves. Also, local sources (sinks) of wave activity are indicated by the local divergence (convergence) of the Plumb flux. Thus, this diagnostic is ideal for diagnosing the regional forcing and propagation characteristics of large-scale quasistationary Rossby waves. Furthermore, upon zonal averaging, the meridional and vertical components of the Plumb flux reduce to the EP flux associated with the quasi-stationary waves (Plumb 1985). This allows a direct assessment of the contribution of a particular quasi-stationary wave field to the zonal mean wave driving described in eqt 2.10.

The Plumb flux is used to study the dynamical nature of large-scale quasistationary eddies contained within the composite NAM anomaly fields (such coherent planetary scale eddies are distinguished from incoherent contributions related to case-tocase variability). A brief comment on coherent versus incoherent eddies is in order before progressing further. As an example, consider the basic composite procedure described in section 2.3. In the TEM framework, we need the composited eddy flux given by $\left\langle u^{\prime} v^{\prime}\right\rangle$ where the brackets represent the composite operator. Compositing the individual terms of this flux, $\left\langle u^{\prime}\right\rangle\left\langle v^{\prime}\right\rangle$, gives the coherent eddy flux component (the flux due to the composite anomalies), while the difference $\left\langle u^{\prime} v^{\prime}\right\rangle-\left\langle u^{\prime}\right\rangle\left\langle v^{\prime}\right\rangle$ represents the incoherent eddy flux (due to case-to-case variability).

The Plumb flux is used to assess (a) regional wave sources and 3-D wave 
propagation and (b) the net contribution of the coherent eddy field to the zonal mean wave driving of the TEM budget. Since eqt 2.17 is nonlinear with respect to the quasistationary eddy field, one must take care in constructing the most relevant flux anomaly field, noting that the climatological-mean stationary waves also produce a substantial Plumb flux contribution. To correctly diagnose the net impact of the coherent anomaly fields upon the total Plumb flux field, one must take the difference between the Plumb flux calculated from (i) the total quasi- stationary wave field (anomalies plus climatological values) and (ii) the climatological stationary wave field. This approach is distinguished from a calculation based solely upon the composite quasi-stationary wave anomalies and is more relevant in assessing the net impact upon the zonal mean tendency dynamics.

\subsection{Potential Vorticity Inversions}

To further investigate the influence of different domains (e.g. troposphere, stratosphere) on the overall circulation, potential vorticity (PV) inversions are performed. Consider PV in a rotating cylinder of fluid. The tube will have absolute (total) vorticity related to the sum of its relative (rate of rotation) and planetary (latitude dependent) vorticity. Now, if we squeeze the cylinder adiabatically so that the cross section of the rotating fluid decreases, by conservation of mass (assuming a quasi- incompressible fluid) the cylinder must lengthen. By conservation of angular momentum, if the radius of the cylinder decreases the rate of rotation must increase. Therefore, the spin and effective depth of the fluid parcel are related with the ratio of the two being the column's potential 
vorticity.

Mathematically, PV can be represented by various formalisms based on the

problem being considered. Using the quasi-geostrophic framework, the balance condition for potential vorticity anomalies in spherical geometry can be written as:

$$
q^{*}=\frac{1}{f^{2}}\left[\frac{1}{(r \cos \phi)^{2}} \frac{\partial^{2}}{\partial \lambda^{2}}+\frac{f}{r^{2} \cos \phi} \frac{\partial}{\partial \phi}\left(\frac{\cos \phi}{f} \frac{\partial}{\partial \phi}\right)+f^{2} \frac{\partial}{\partial p}\left(\frac{1}{\sigma} \frac{\partial}{\partial p}\right)\right] \Phi^{*}
$$

where $q$ is the potential vorticity and $\sigma$ is a horizontally invariant measure of the static stability (Black 1998)

Piecewise potential vorticity inversion is the procedure of inverting eqt 2.19 for a specific subset of the 3-D PV anomaly distribution. The procedure is performed as follows: (i) The overall $q^{*}$ distribution is calculated from the geopotential anomaly field.

(ii) Specific anomalies or domains are then selected and inverted to obtain the circulation induced by only those specific features. In practice, this allows an assessment of the role of stratospheric PV anomalies in influencing tropospheric circulations. Because of its elliptic character, the circulation response $\left(\Phi^{*}\right)$ to a localized PV anomaly is a field that decays vertically with a scale $H=f L / N$ where $L$ is the horizontal scale of the anomaly and $N$ is the Brunt-Väisälä frequency (Black and McDaniel 2004) . Therefore, a stratospheric PV anomaly structure with large magnitude but small horizontal extent will have less influence at the surface than an anomaly structure with less absolute magnitude but large horizontal extent. A further application of eqt 2.19 is to perform piecewise PV - tendency inversions. In this case the time derivative of the geopotential anomaly field is used to 
calculate the PV-tendency field. Specific features in the PV-tendency field are then inverted to yield the zonal wind tendency distribution attributable to the PV anomaly

feature of interest. The zonal wind tendencies obtained are useful in assessing the role of specific features in the overall budget described in eqt 2.10 . 


\section{CHAPTER 3}

\section{REGRESSION ANALYSES}

\subsection{General Structure}

Regressions based on the procedure outlined in chapter 2 are used for identifying the canonical patterns associated with the Northern Annular mode. To assess the mean NAM structure (versus assessing the structure at a particular stage of development), we average over the entire 21 day time evolution (details of the 21 day evolution can be found in section 2.2). By averaging over time, direct comparisons can be made with the NAM structures found by Thompson and Wallace $(1998,2000)$ and Baldwin and Dunkerton (1999).

The top panel of figure 3.1 shows the mean NAM 1000mb geopotential height anomaly pattern (comparable to TW98 sea level pressure patterns) while the bottom panel displays the zonally averaged zonal wind anomaly field for the northern hemisphere. Examining the height anomaly pattern first, the pattern is dominated by a north-south seesaw of atmospheric mass between high latitudes and sectors of the midlatitudes, primarily the Atlantic and Pacific basins. The minimum (or largest) negative height anomalies are shifted away from the pole and are centered over the north Atlantic while the largest positive anomalies are located over the northeastern Atlantic and western Europe.

Consistent with the North-South seesaw pattern found in the height anomaly field, the zonal wind anomaly field is characterized by a meridional dipole centered around 

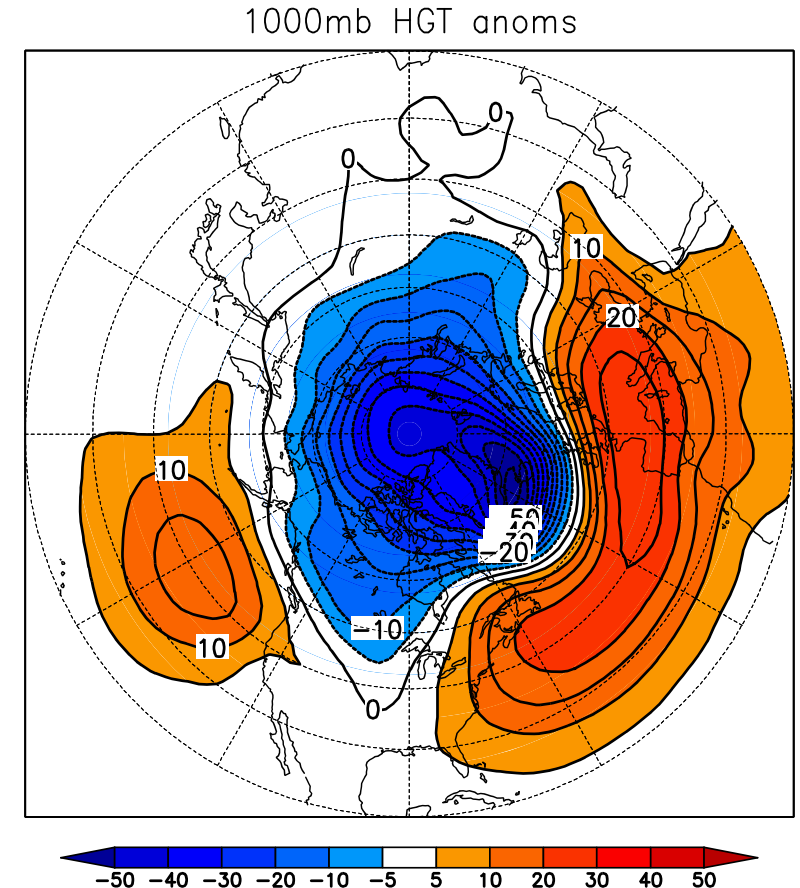

b

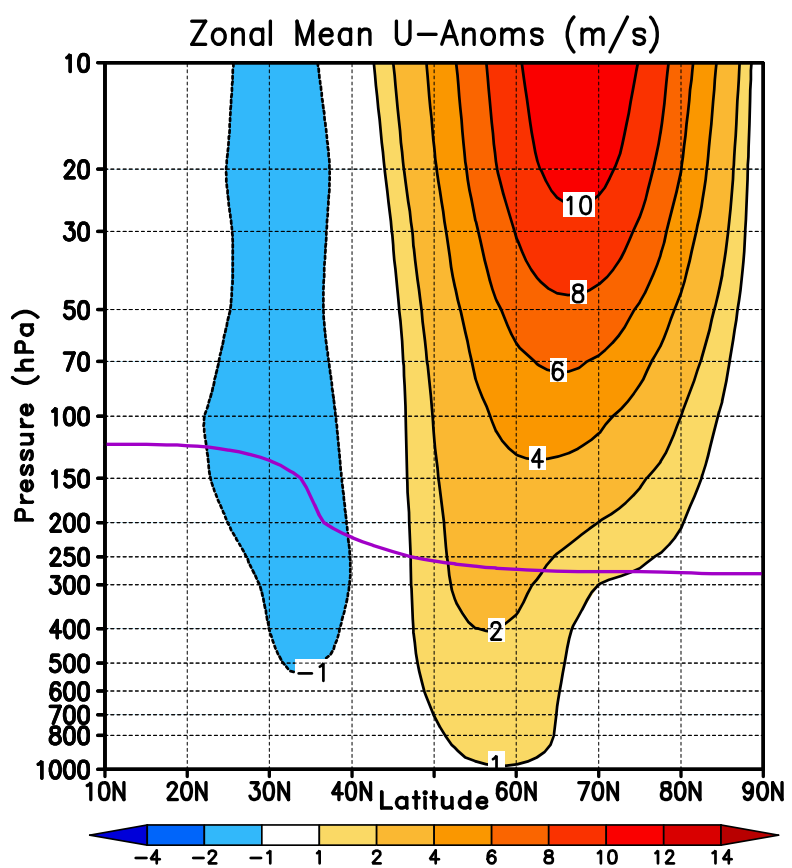

Figure 3.1 Mean structure of the NAM produced by averaging all 21 lag regressions. Figure (a) shows the $1000 \mathrm{mb}$ geopotential height anomalies while figure (b) shows a latitude-height cross section for the zonally averaged zonal wind anomalies. 
$45 \mathrm{~N}$ (figure 3.1, bottom panel). The anomalous winds are characterized by deep, vertically coherent westerlies (easterlies) at higher (lower) latitudes. High latitude westerly wind anomalies extend from the surface well into the stratosphere, achieving maximum amplitudes in the middle stratosphere. The high-latitude anomaly field is also characterized by a poleward tilt with height between the troposphere and lower stratosphere. The easterly wind anomalies, centered at $32.5 \mathrm{~N}$, have both a stratospheric and tropospheric component but do not have a substantial signature at the surface.

\subsection{Lag-Regression Analyses}

Using the procedure outlined in section 2.2, one can examine the time evolution of the NAM signal as it descends from the stratosphere to the surface. To consolidate the results to represent different stages in the evolution for presentation purposes, 6 bins have been used when presenting the results: days 1 to 4,5 to 8,9 to 12,13 to 15,16 to 18 , and 19 to 21 . The latter 3 bins are taken to be 3 days (as compared to 4 days for the first 3 bins) due to the increased rate of descent of the NAM signal once it reaches the troposphere (as seen in figure 2.1).

Figures 3.2 - 3.4 display northern hemisphere polar stereographic plots of geopotential height anomalies at $10 \mathrm{mb}, 300 \mathrm{mb}$, and $1000 \mathrm{mb}$ respectively which corresponds to stratospheric, upper tropospheric, and near surface levels. Looking first at the early stages of the $10 \mathrm{mb}$ evolution displayed in figure $3.2 \mathrm{a}-\mathrm{c}$, an approximately zonally symmetric dipole is seen with large negative anomalies centered over the pole and weaker positive anomalies at midlatitudes. The anomalous low centered over the 
a

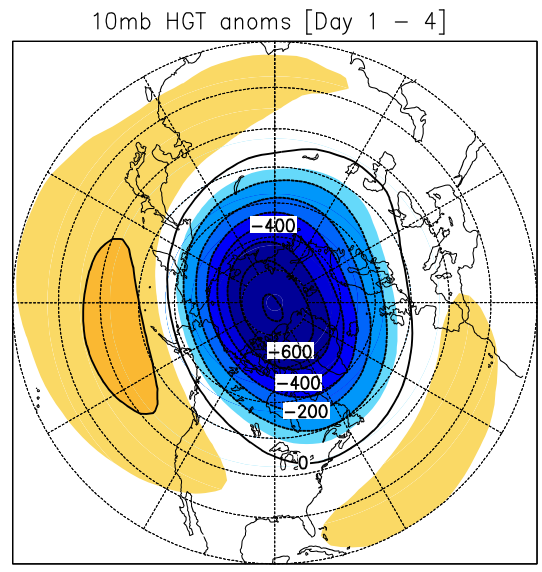

c

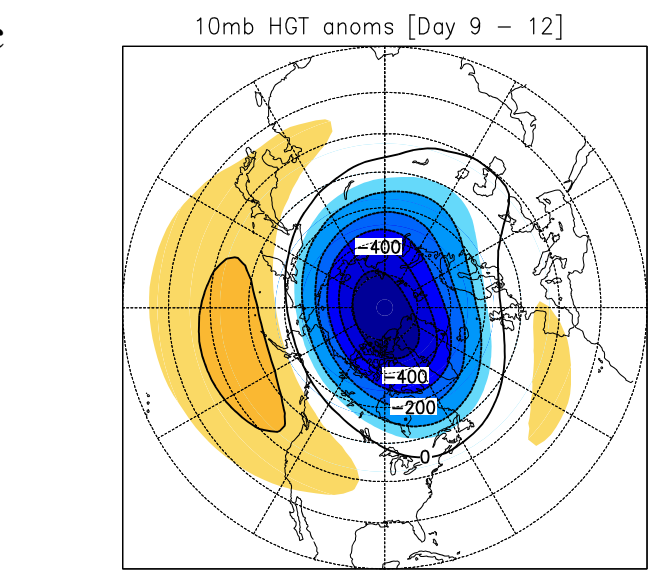

e

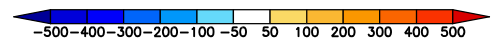

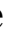

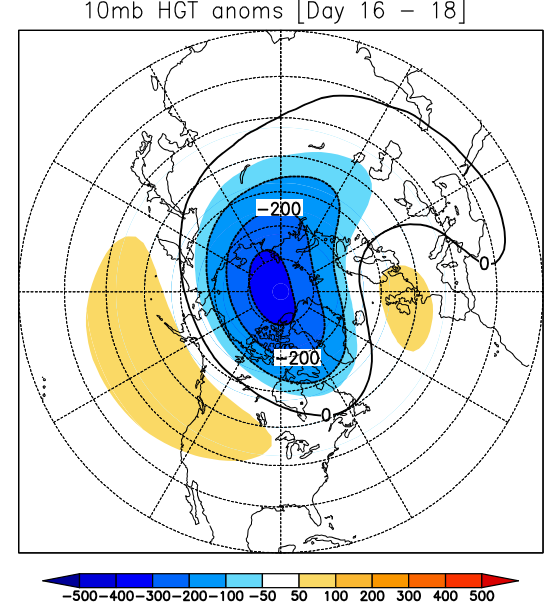

b
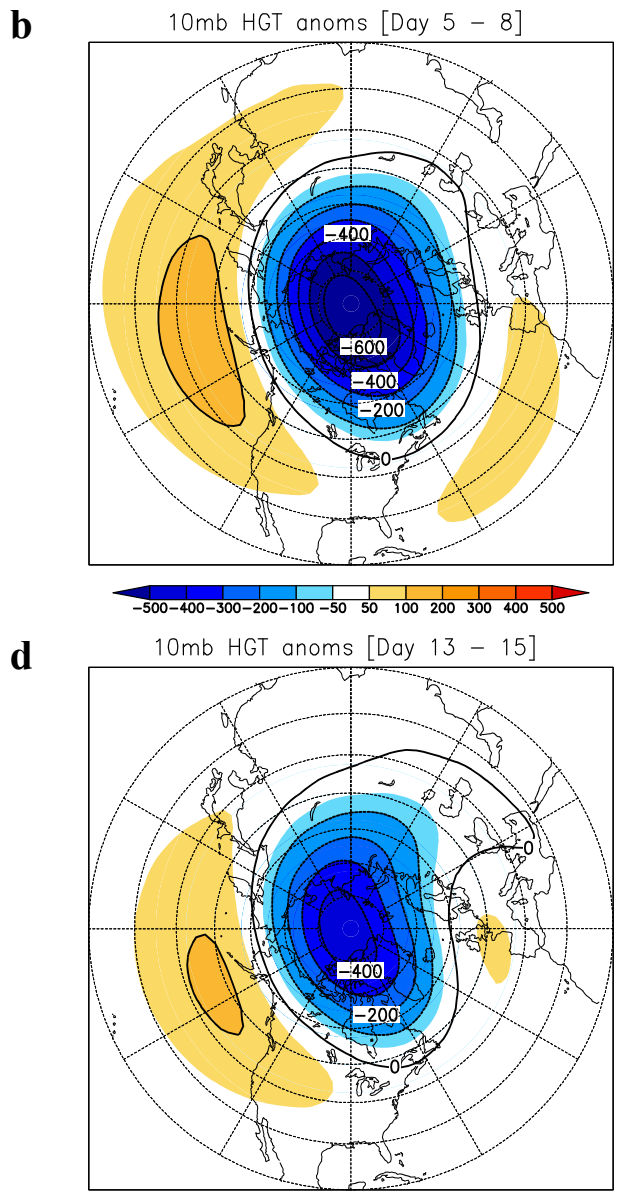

$-500-400-300-200-100-50 \quad \frac{1}{50} \quad \frac{1}{100} 200 \quad \frac{10}{300} 400 \quad 500$

f

$10 \mathrm{mb}$ HGT anoms [Day $19-21]$

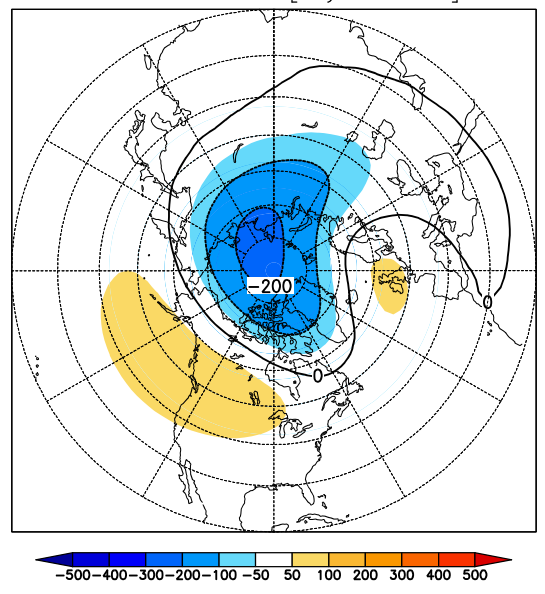

Figure 3.2 Polar stereographic plots for the regressed $10 \mathrm{mb}$ geopotential height anomalies for days (a) 1 - 4, (b) 5 - 8, (c) 9 - 12, (d) 13 - 15, (e) 16 - 18, and (f) $19-21$. See text (section 2.2) for details on the regression and mapping to a 21 day time axis. Units are geopotential meters. 
pole shows a progressive weakening as the NAM signal progresses from the middle stratosphere into the troposphere (figures 3.2a-f) but the overall structure remains constant. The positive anomaly feature, displaying two distinct maxima in the early stages of development (one over the Pacific basin and one over the North Atlantic), also weakens as the signal descends, losing some of its annular character. As the signal nears the surface (figure 3.2f), the north Atlantic center has all but disappeared while the pacific center has weakened to less than $100 \mathrm{~m}$.

The evolution of the NAM pattern in the upper troposphere is displayed in figure 3.3. Again the seesaw pattern between the midlatitudes and polar region is evident at all stages of development. The negative anomaly, centered over the pole in the stratosphere (figure 3.2), has shifted to the southern tip of Greenland. Another weaker negative center develops over the Aral sea, eventually becoming connected to the polar low. The extent of the midlatitude positive anomaly has increased from that seen in the stratosphere, covering most of the northern Pacific and Atlantic basins. Two separate positive anomaly maxima are found over both ocean basins. The $300 \mathrm{mb}$ height anomalies remain large even after the NAM signal has peaked at $300 \mathrm{mb}$ and descended downward (figures 3.3e and 3.3f), illustrating the robustness of the late stage tropospheric NAM pattern and its vertical coherence.

Examining the surface signature of the NAM in figure 3.4, the negative anomaly maximum is again shifted away from pole and centered southeast of Greenland indicating a westward tilt with height. This negative anomaly feature continues to strengthen throughout panels a - $\mathrm{f}$ as the signal descends, as does the positive anomaly maxima located over northeastern Europe and the western Pacific. Unlike the upper tropospheric 
a

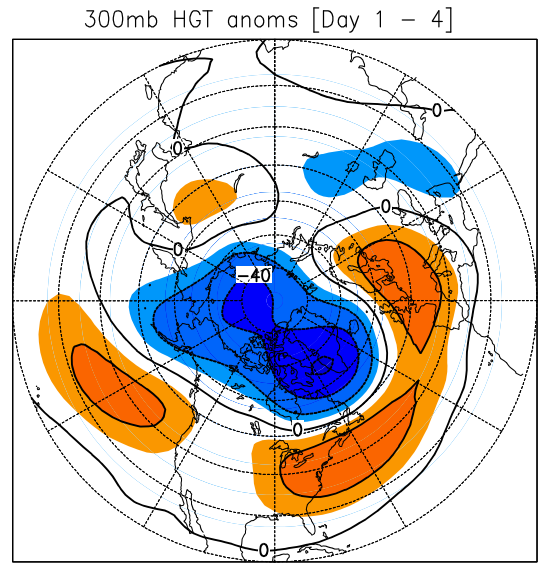

c

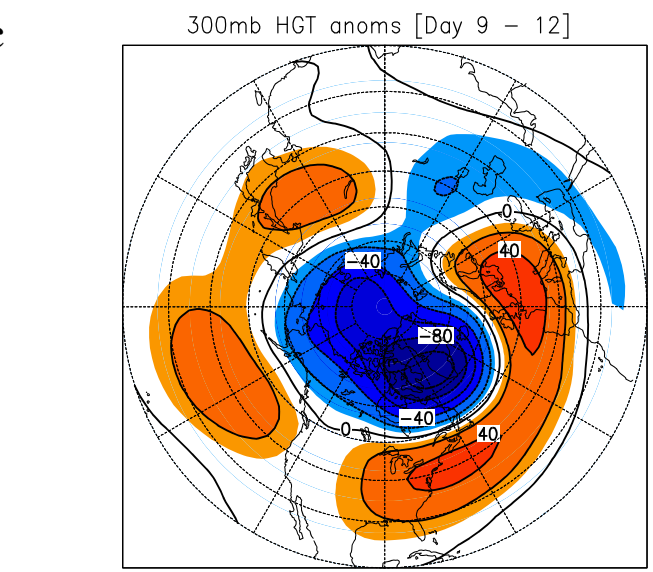

e

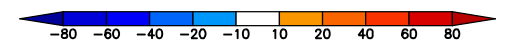

$300 \mathrm{mb}$ HGT anoms [Day 16 - 18]

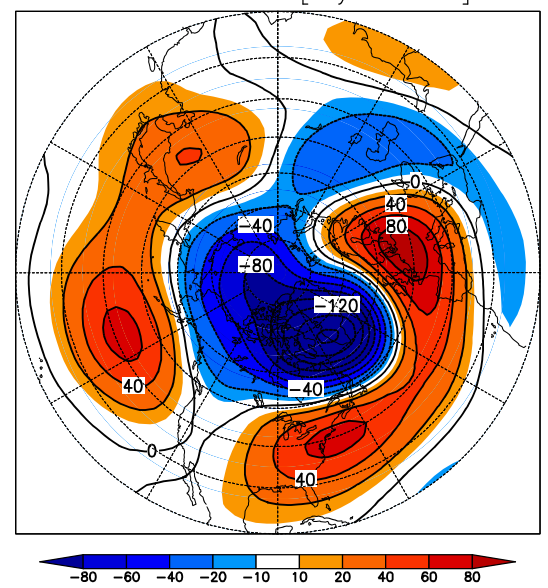

b

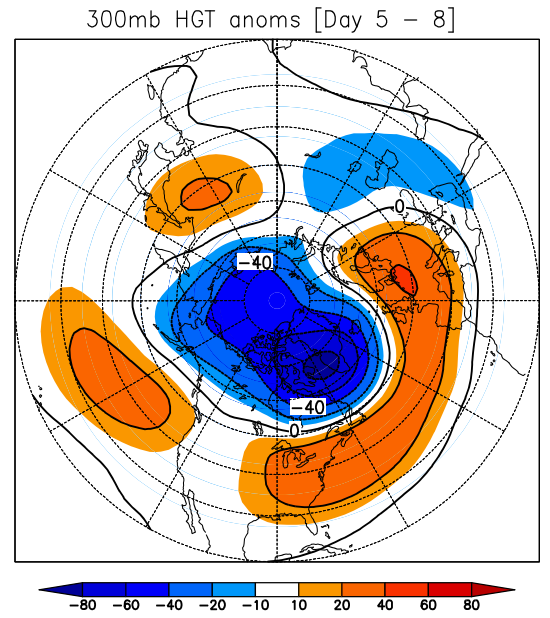

d

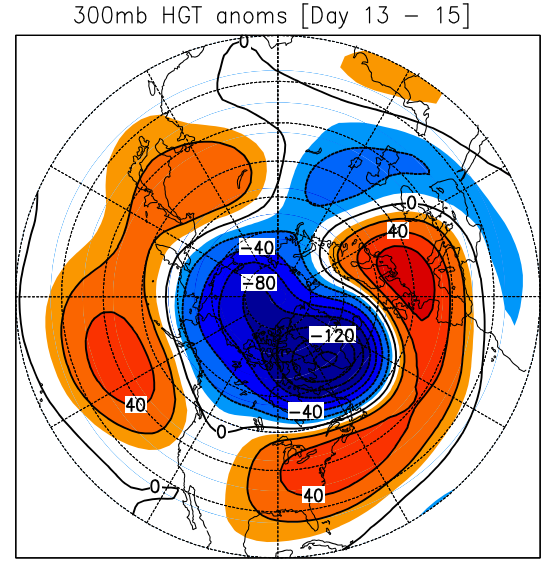

f

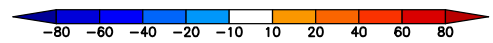

$300 \mathrm{mb}$ HGT anoms [Day $19-21]$

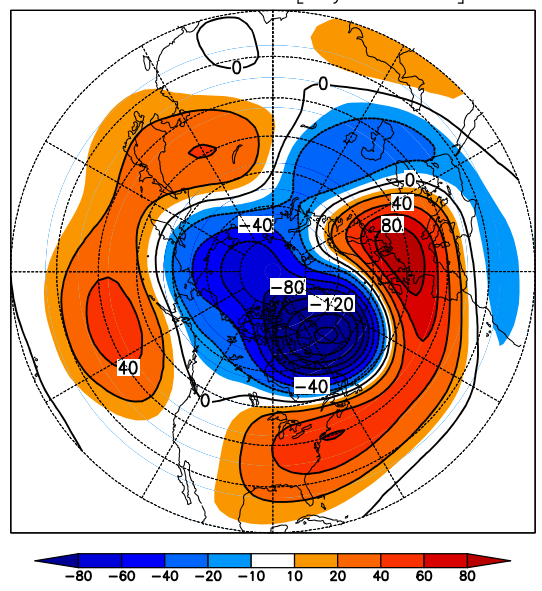

Figure 3.3 Polar stereographic plots for the regressed $300 \mathrm{mb}$ geopotential height anomalies for days (a) 1 - 4, (b) 5 - 8, (c) 9 - 12, (d) 13 - 15, (e) 16 - 18, and (f) $19-21$. See text (section 2.2) for details on the regression and mapping to a 21 day time axis. Units are geopotential meters. 
a

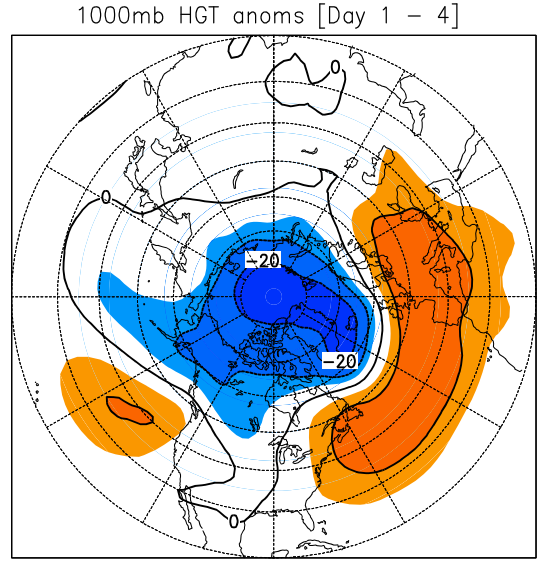

c
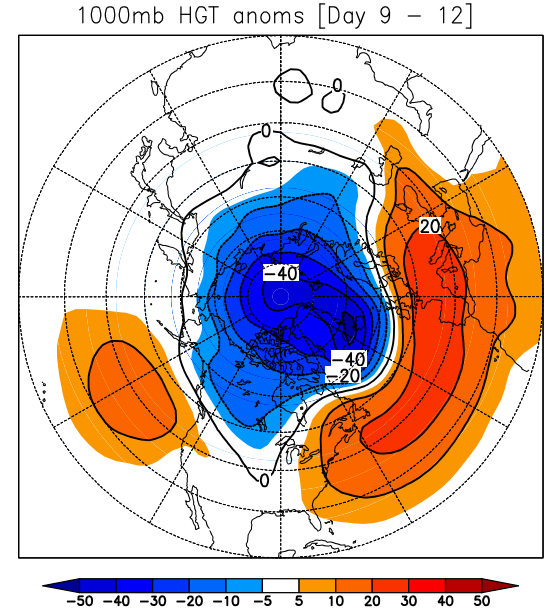

$1000 \mathrm{mb}$ HGT anoms [Day $16-18$ ]

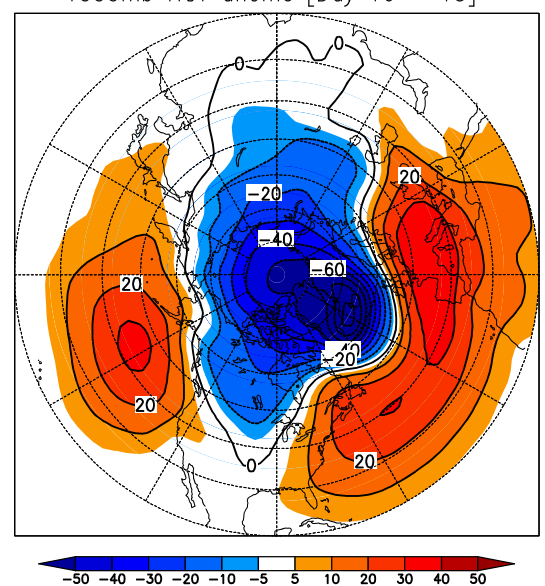

b

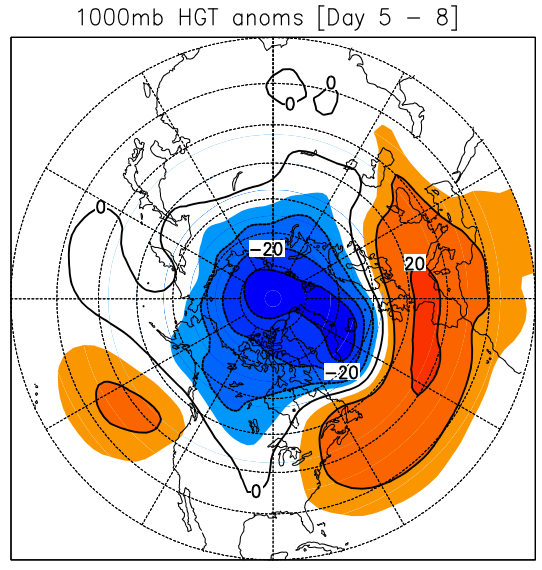

d

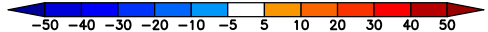

$1000 \mathrm{mb}$ HGT anoms [Day $13-15$ ]
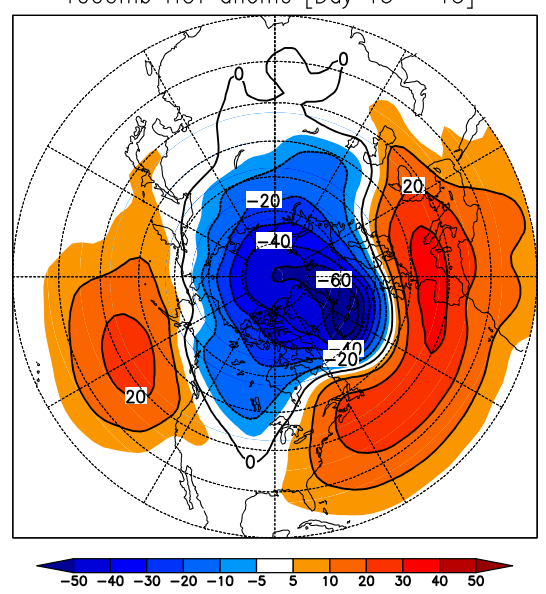

f

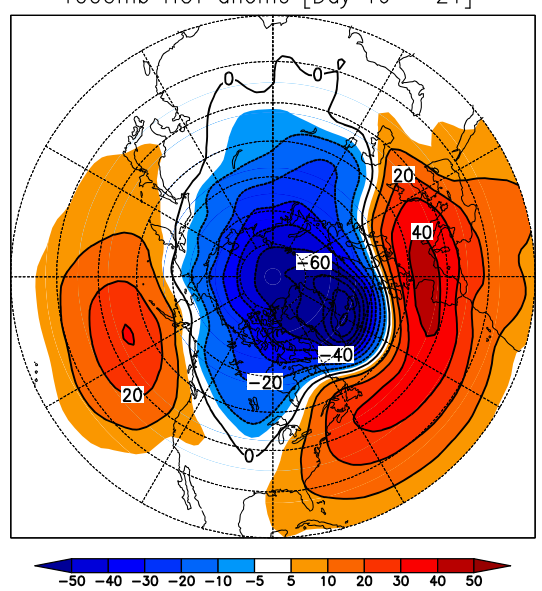

Figure 3.4 Polar stereographic plots for the regressed $1000 \mathrm{mb}$ geopotential height anomalies for days (a) 1 - 4, (b) 5 - 8, (c) 9 - 12, (d) 13 - 15, (e) 16 - 18, and (f) 19 -21. See text (section 2.2) for details on the regression and mapping to a 21 day time axis. Units are geopotential meters. 
midlatitude patterns, the $1000 \mathrm{mb}$ pattern displays only one maximum per ocean basin. If we examine the longitudinal position of the positive anomaly feature found in the north Atlantic at later stages of the evolution (i.e. figures $3.3 \mathrm{f}$ and $3.4 \mathrm{f}$ ), the westward tilt with height necessary for Rossby wave propagation is clearly evident. This is likely the conduit for vertical energy propagation from near the surface to the upper troposphere (and perhaps the lower stratosphere). Thus, even though the signal moves downward, it is accompanied by upward Rossby wave energy propagation (at later stages) in the troposphere. In the Pacific, the structure is more equivalently barotropic with little tilt in either direction.

By examining the zonally averaged zonal wind anomaly field, we can more concisely characterize information at multiple levels and descent stages. Figure 3.5 illustrates the descent of zonal wind anomalies at the 6 temporal bins described above. For days 1-4, an anomalously strong polar vortex is observed with little tropospheric influence. A weaker negative anomaly is centered at $\sim 30 \mathrm{~N}$, vertically spanning the stratospheric domain. For days 5-12, both the high-latitude and midlatitude zonal anomaly patterns have descended into the troposphere while the maximum stratospheric winds have begun to weaken. Even as early as day 9 , the $1 \mathrm{~m} / \mathrm{s}$ anomalies have already reached the surface. For days 13-21, the stratospheric maximum continues to weaken while the tropospheric component strengthens with the $2 \mathrm{~m} / \mathrm{s}$ contour reaching nearly to the surface in the final panel. The meridional area impacted by the positive anomalies in the troposphere also increases, stretching from $\sim 47 \mathrm{~N}$ to near $80 \mathrm{~N}$. The upper stratospheric component of negative anomaly feature observed at lower latitudes experiences uniform weakening as time progresses. This features strengthens near the 
a

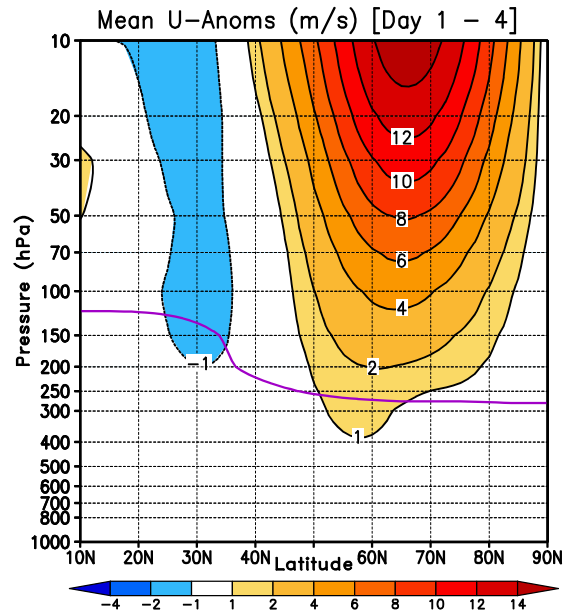

c

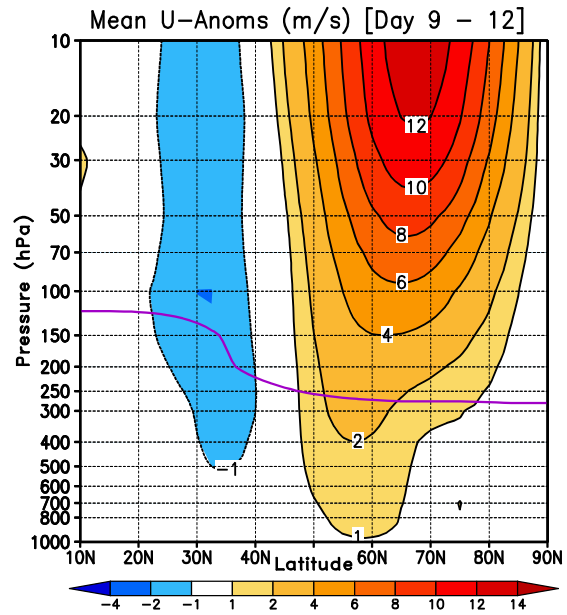

e

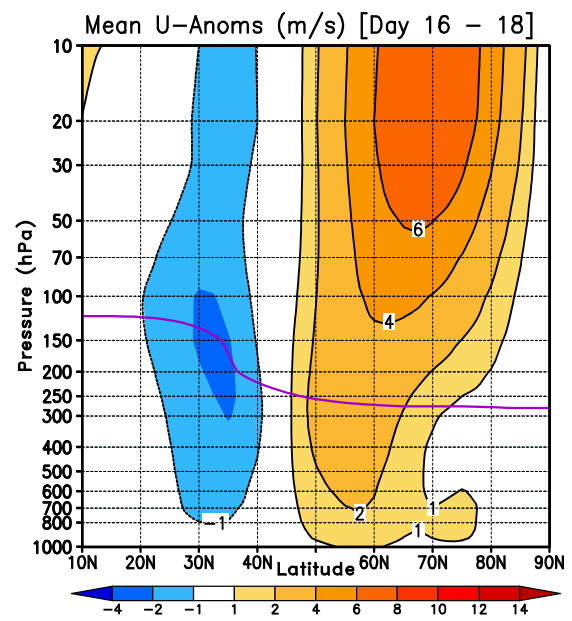

b

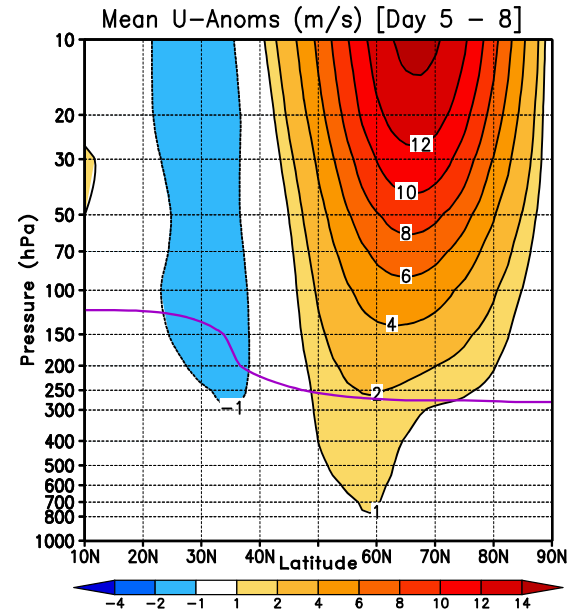

d

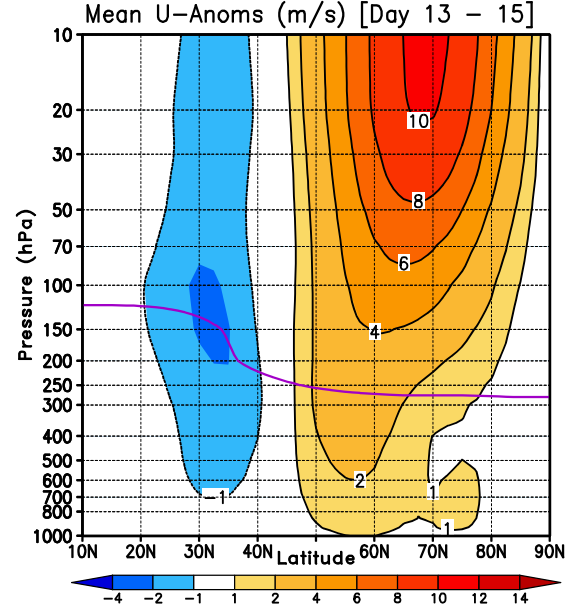

f

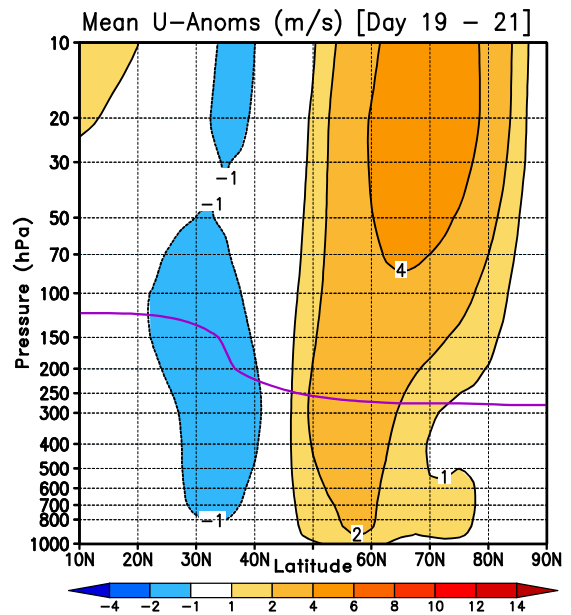

Figure 3.5 Zonally averaged zonal wind anomalies [m/s] for days (a) 1 - 4, (b) 5 - 8 , (c) 9 - 12, (d) 13 - 15, (e) 16 - 18, and (f) $19-21$. 
tropopause, reaching maximum amplitudes at days 13-18 and begins to weaken in the final panel.

The transformed Eulerian mean diagnostic analyses are displayed in figure 3.6. Due to the slowly varying nature of these components during the NAM descent, only 2 stages are presented for brevity. In the upper panels (days 9 - 12), the anomalous EP flux and associated wave driving and the Coriolis torque are plotted. ${ }^{1}$ The anomalous EP flux pattern is characterized by equatorward and upward fluxes in the high latitude troposphere $(\sim 65 \mathrm{~N})$ and equatorward and downward propagation in the midlatitude troposphere. The upper stratosphere is characterized by EP flux convergence at high latitudes, as seen by the negative wave driving (slowing of the polar vortex). The Coriolis torque acts to partially offset this wave driving and restore thermal wind balance, as seen by the oppositely signed features in figure $3.6 \mathrm{~b}$.

In the bottom panels where the NAM signal has fully reached the troposphere, the tropospheric EP flux is now characterized by divergence around $75 \mathrm{~N}$, leading to an acceleration in the zonal wind field. The changes in the EP flux pattern from 3.6a to 3.6c that lead to the tropospheric flux divergence occur in the lower troposphere as some of the upwardly propagating EP flux seen earlier has now shifted to propagate toward the surface. The high latitude stratospheric convergence observed in figure 3.6a is still present but has weakened. The Coriolis torque continues to act to counteract the wave

${ }^{1} \mathrm{EP}$ flux vectors are scaled to point in the proper direction (the meridional component is divided by 225 to account for plot aspect ratio) while enhancing magnitudes at successively higher altitudes (both meridional and vertical components are multiplied by $\left.e^{z / H}\right)$. Although the local EP flux divergence is not preserved with this scaling, the flux divergence can be directly inferred from the wave driving field. This issue is discussed further in Baldwin et al. (1985). 

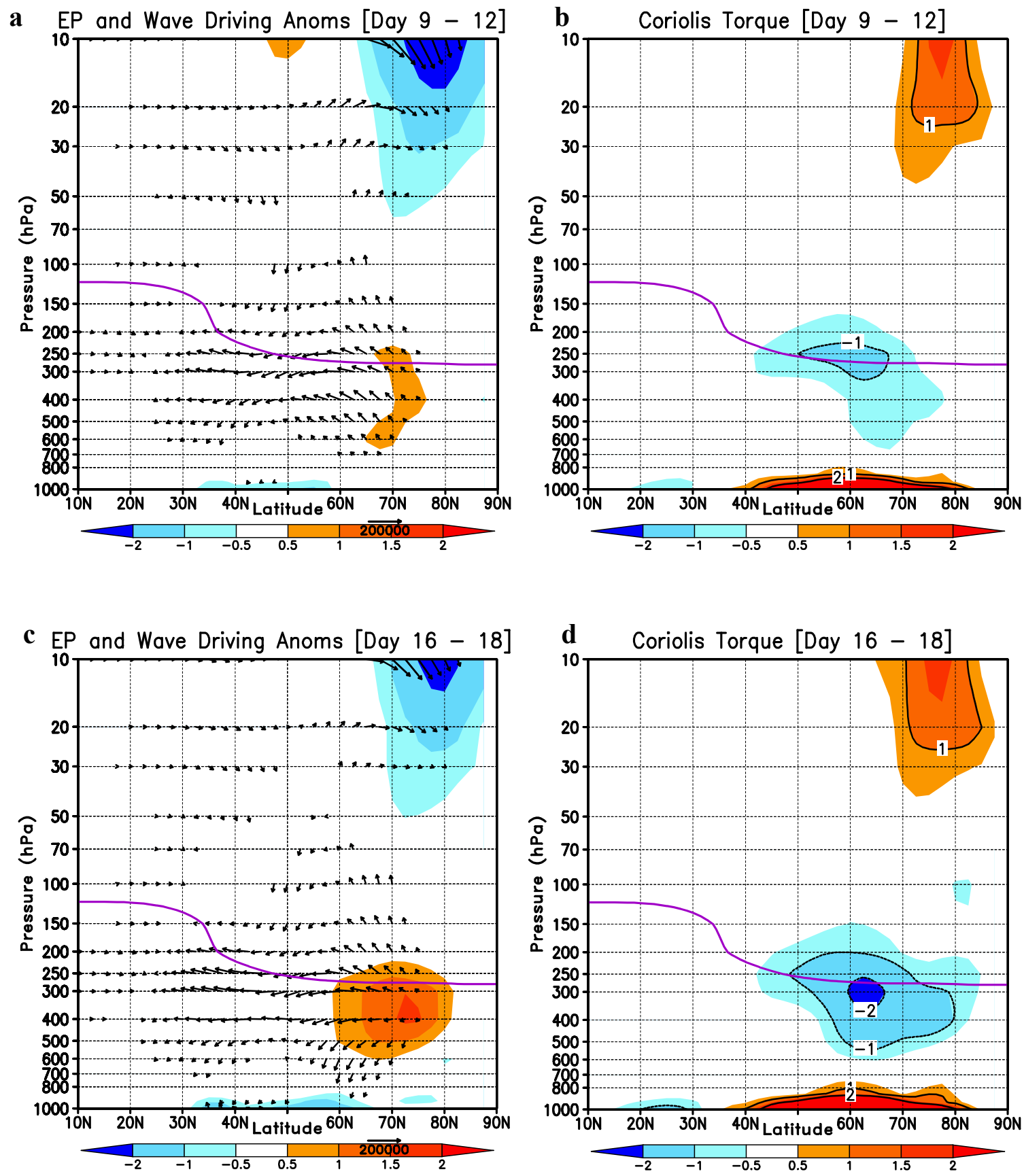

Figure 3.6 Anomalous EP flux vectors $\left[\mathrm{m}^{3} \mathrm{~s}^{-2}\right]$ and wave driving $[\mathrm{m} / \mathrm{s} /$ day $]$ for days (a) 9 - 12 and days (c) 16 - 18 and the Coriolis Torque (fv ${ }^{*}$ ) [m/s/day] for days (b) 9 - 12 and days (d) 16 - 18. EP flux vectors are scaled to point in the proper direction while enhancing magnitudes at successively higher altitudes (see text for details). The local EP flux divergence is not preserved but can be inferred from the wave driving. 
driving, resulting in a first order balance between the two terms. Due to its relatively small magnitude, the zonal wind tendency (approximately the sum of the wave driving and Coriolis torque) is not shown. We note that the Coriolis torque term acts to spread the upper tropospheric acceleration to the lower boundary at high latitudes.

The lack of a substantial wind tendency for each lag can be explained by examining figure 3.7 which shows the $150 \mathrm{mb}$ NAM index time series composited with respect to peak magnitude values for all events exceeding one standard deviation. [The vertical level of the NAM index composited has little affect on the overall structure displayed in figure 3.7.] Figure 3.7 shows that NAM events typically experience prolonged periods of monotonic growth and decay, each lasting 10-12 days. During periods of growth (before the maximum), the winds are accelerating (positive wind tendency) while periods of decay (after the peak) experience a deceleration. Because the regression operator samples both of these phases (growth and decay) non preferentially, the regressed wind tendency in this approach is negligible. The wave driving observed at the earliest stages of development (not shown) is negative in the upper, high latitude stratosphere (as in figure 3.6a). This is due to the regression operation emphasizing periods when the signal is at a maximum. Because of this, the NAM signal at days 1-4 represents a period where the NAM signal is peaking and the eddies are acting to decelerate the zonal flow back to its long term mean. The composites described in chapter 5 identify periods of growth or decay separately, yielding a more dynamically interesting result. 


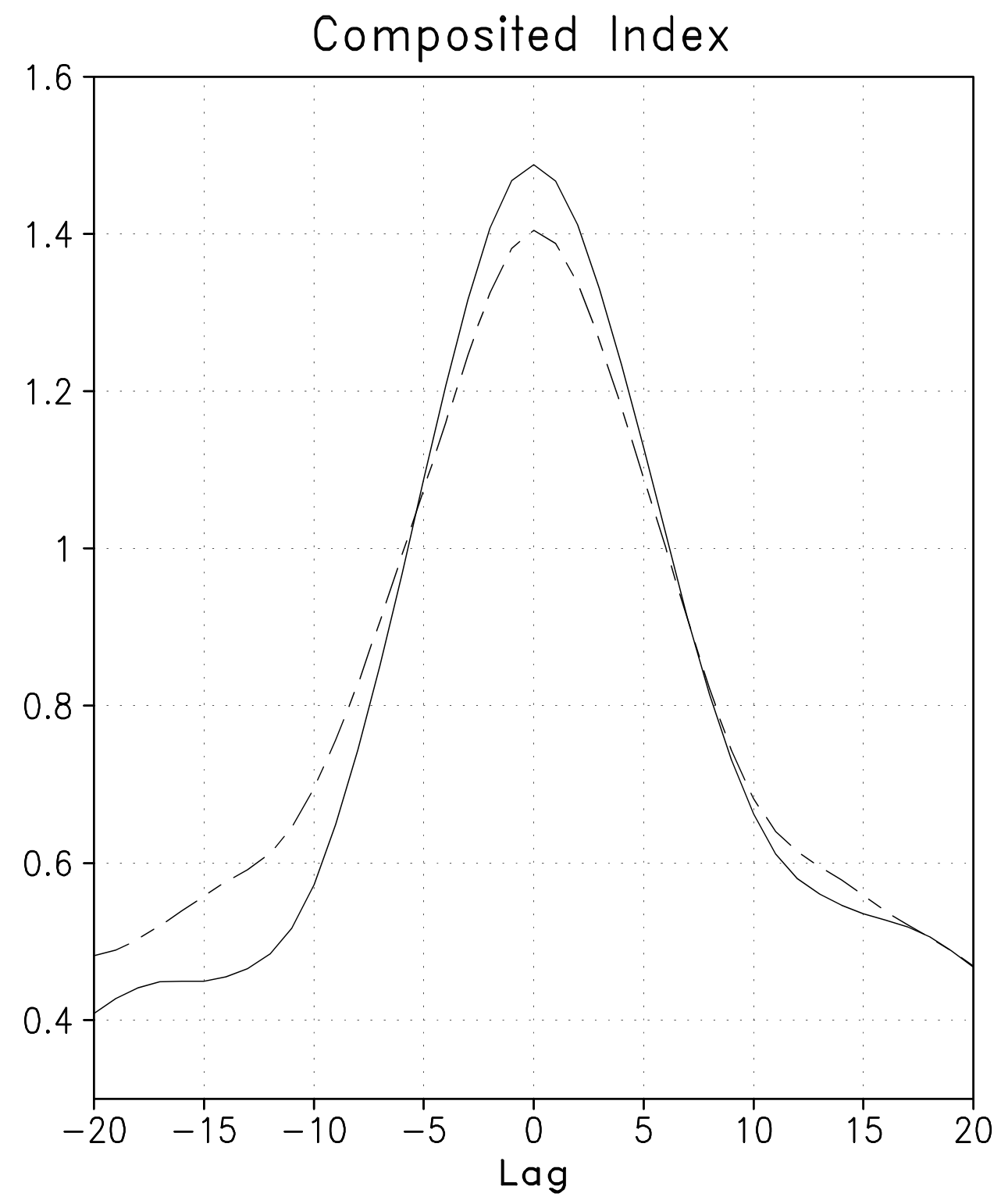

Figure 3.7 Composite of the smoothed NAM index based on its maximum value. The solid line represents the positive phase of the NAM while the dashed line represents the negative phase times $(-1)$. 


\subsection{Summary}

The NAM is characterized by a zonally-symmetric see-saw in atmospheric mass and a corresponding vertically coherent dipole in the zonally averaged zonal wind field. The canonical surface NAM pattern features significant geopotential height anomalies over the pole and midlatitude ocean basins but only weak anomaly features over North America and Eastern Europe/Asia. As one examines the lag 0 NAM pattern at progressively higher altitudes, the zonal asymmetries lessen and the pattern becomes more annular. The negative maximum, centered over the North Atlantic at tropospheric altitudes, shifts poleward in the stratosphere. The NAM pattern appears robust with the same structures resulting from different analysis techniques.

The propagation of the NAM signal from the middle stratosphere to the surface takes approximately 21 days. During this descent, the NAM signal becomes increasingly robust at lower elevation while at upper levels the NAM pattern loses coherence and magnitude. Anomalous EP fluxes and the corresponding wave driving act to decelerate the stratosphere throughout the descent time period while, for the second half of the descent period, act to accelerate the troposphere. The anomalous wave driving thus helps provide the zonal acceleration needed to give rise to the tropospheric zonal wind anomalies present at later stages. 


\section{CHAPTER 4}

\section{CASE STUDIES}

\subsection{Methodology and Case Statistics}

During the average Boreal winter, the polar vortex experiences periods of warming and cooling. At stratospheric levels the NAM index can be used as a proxy for these events. The troposphere also experiences periods of increased (decreased) zonal wind during the course of an average winter with these events projecting onto the tropospheric NAM. However, not all NAM cases follow the statistical prototype of stratospheric initiation followed by downward signal propagation into the troposphere, as certain robust stratospheric NAM events are not associated with corresponding succeeding tropospheric events (Baldwin and Dunkerton 1999; Zhou et al. 2002). Accounting for such case to case variability represents an important test of any theory regarding stratospheric influence upon tropospheric climate.

Figure 4.1 (Plate 1, Baldwin and Dunkerton 1999) shows the height-time development of the NAM index time series for 40 years of data. Figure 4.1 illustrates that both the troposphere and stratosphere are characterized by large-scale cooling and heating events, plotted in blue and red respectively, with events occurring regularly during the Boreal winter months. Examining the intraseasonal behavior found during a given year, one can easily see that substantial variability exists between events. Our approach is to examine different types of NAM case evolutions with a goal of identifying dynamical reasons for the variability among different case developments. 

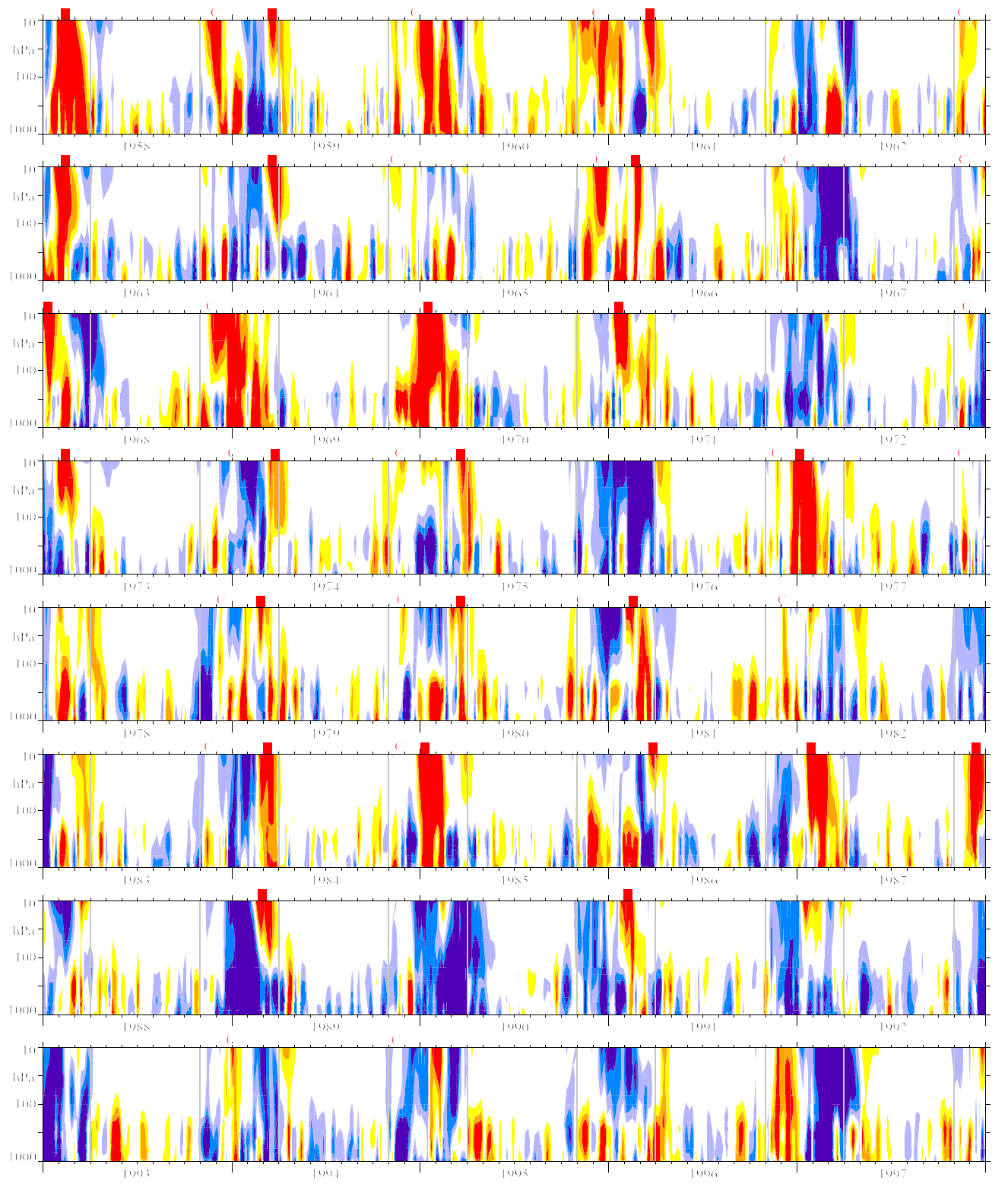

Figure 4.1 10-day low-pass filtered NAM signature time series for 1958-1997. Contour values between -0.6 and 0.6 are not shaded. Red corresponds to a weak, warm polar vortex while blue corresponds to a strong, cold polar vortex. Faint vertical lines denote the November-March winter season. Red tick marks indicate major midwinter or early final warnings during DJFM. Figure provided by Mark Baldwin. 
The focus of the case studies is on the study of robust deep stratospheric NAM events stratified according to whether or not they are followed by like signed tropospheric NAM events. Cases are identified from normalized, multi-leveled low-pass NAM indices (i.e. figure 4.1). The case selection methodology begins by identifying intraseasonal time periods during the Boreal winter that are characterized by $10 \mathrm{mb}$ NAM index anomaly magnitudes greater than $1.5 \sigma .{ }^{1}$ This selects robust mid-stratospheric events. We then require that each $10 \mathrm{mb}$ event be followed by a subsequent (within 10 days) like-signed NAM index anomaly at $100 \mathrm{mb}$ with magnitudes greater than $0.5 \sigma$. This ensures a deep stratospheric NAM event extending into the lower stratosphere. Finally, we categorize deep stratospheric NAM events according to whether or not the $100 \mathrm{mb}$ NAM signal is succeeded (within 10 days) by a like-signed NAM index anomaly signature at $500 \mathrm{mb}$ having magnitudes of $0.5 \sigma$ or greater. The effectively separates events primarily confined to stratospheric altitudes ("nonpropagating events") from those with substantial downward extensions into the troposphere ("propagating events"). It is important to emphasize that propagating and nonpropagating refer to the apparent vertical movement of the NAM signal downward and do not imply a true geophysical wave propagation.

Using the approach, we identify 16 positive stratospheric NAM events (westerly anomalies therefore a strengthened polar vortex) and 26 negative stratospheric NAM events (easterly anomalies thus a weakened polar vortex) that occur during the 40 year period 1958-1997. About $30 \%$ of these 42 cases ( 7 positive, 6 negative) are

${ }^{1}$ Like-signed $10 \mathrm{mb}$ events are considered distinct if they are separated by from one another by at least 10 days. 
nonpropagating events that remain confined to stratospheric altitudes. We first present diagnostic results for three individual cases in order to illustrate some basic aspects of case-to-case variability. Case 1 is a positive NAM event occurring during February 1976 that is observed to propagate into the troposphere. Case 2 (case 3) represents a negative NAM event occurring during March 1989 (January 1985) that does not (does) propagate downward into the troposphere. For downward propagating events we focus our attention on the time period during which the tropospheric zonal wind anomalies develop while for nonpropagating events we focus on the time period during which circulation intensification occurs in the lower stratosphere.

\subsection{Illustrative Case Studies}

Figure 4.2 presents the temporal evolution of the zonal-mean zonal wind anomalies at $60 \mathrm{~N}$ for case 1 (positive, propagating) and case 2 (negative nonpropagating), which represent two opposing tropospheric responses to stratospheric anomalies. As will become evident, case 1 exhibits many of the canonical characteristics of NAM development: The zonal wind anomaly is initially most prominent in the midstratosphere and then strengthens and descends into the lower stratosphere with a clear continuation downward through the depth of the troposphere to the surface (figure 4.2, top panel). The strongest anomaly development in the lower troposphere occurs during the 9-day period between days +2 and +11 . Latitude-height cross sections of the zonal mean anomaly fields are displayed in figure 4.3 for this period. These analyses show clear evidence of the NAM intensification in both the troposphere and stratosphere 


\section{Case 1: $2 / 2 / 76-2 / 22 / 76$}

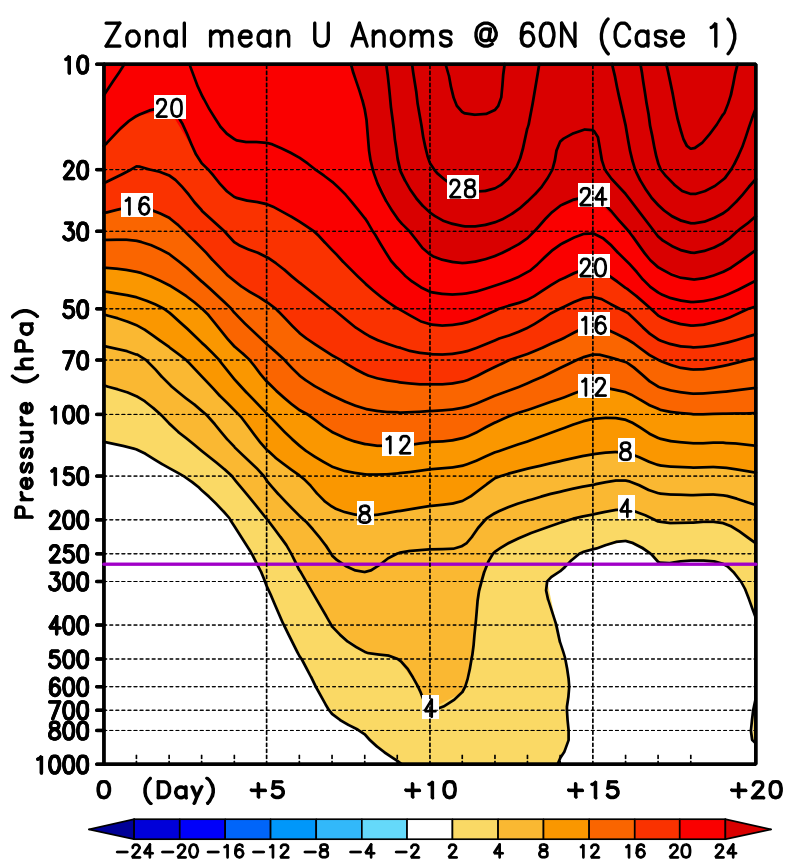

\section{Case 2: 2/26/89 - 3/18/89}

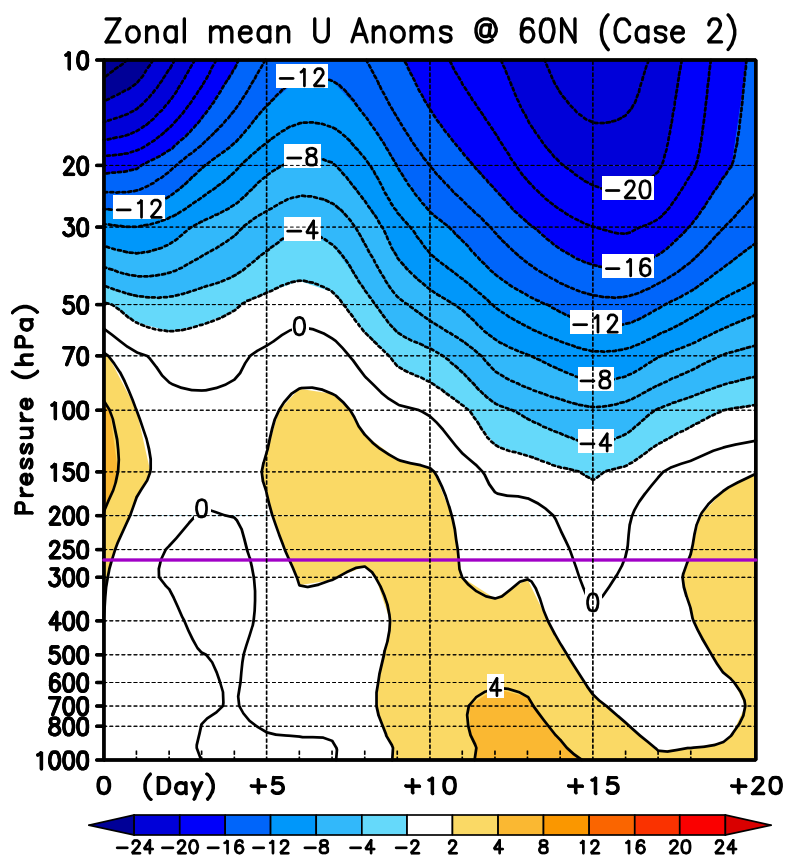

Figure 4.2 Time evolution of the zonal-mean zonal wind anomalies at $60 \mathrm{~N}$ for (upper) case 1 and (lower) case 2 . Units are $\mathrm{m} / \mathrm{s}$. 
(figure 4.3a,b). In particular, we note a coherent pattern of zonal wind intensification over the entire domain depth at midlatitudes (figure 4.3c). ${ }^{2}$ The zonal wind change pattern exhibits the northward tilt with height characteristic of the NAM. In association with the zonal wind changes there is a concomitant north-south dipole structure in the stratospheric PV tendency field with PV increases at high latitudes over much of the stratosphere (figure 4.3d). There are also smaller-scale PV changes evident near the tropopause.

It is of interest to decompose the zonal wind change into separate parts associated with tropospheric and stratospheric PV anomaly features. This is achieved by performing piecewise inversions of the PV change field (figure 4.3d) partitioned by the latitudinally varying tropopause [defined using the WMO thermal tropopause definition (e.g., Lewis 1991)], which is indicated in all plots by the thick purple contour. The piecewise PV inversion results illustrate that zonal wind anomalies induced by the redistribution of stratospheric PV (figure 4.4a) account for the majority of the total stratospheric zonal wind changes (figure 4.3c), as might be expected. However, the stratospheric PV anomalies also provide a first-order contribution to the zonal wind increase observed in the mid-latitude troposphere, locally accounting for as much as $50 \%$ of the observed tropospheric increase. Of course, it is also evident that the tropospheric PV changes also fundamentally influence the observed tropospheric zonal wind changes (figure 4.4b).

The results verify the regression-based results of Black (2002) regarding the direct downward influence of stratospheric PV anomalies during tropospheric NAM

${ }^{2}$ Given the excellent quantitative correspondence between the geostrophic and actual wind fields, we opt to display the geostrophic zonal wind change to facilitate direct comparisons with figures $4.4 \mathrm{a}$ and $4.4 \mathrm{~b}$. 

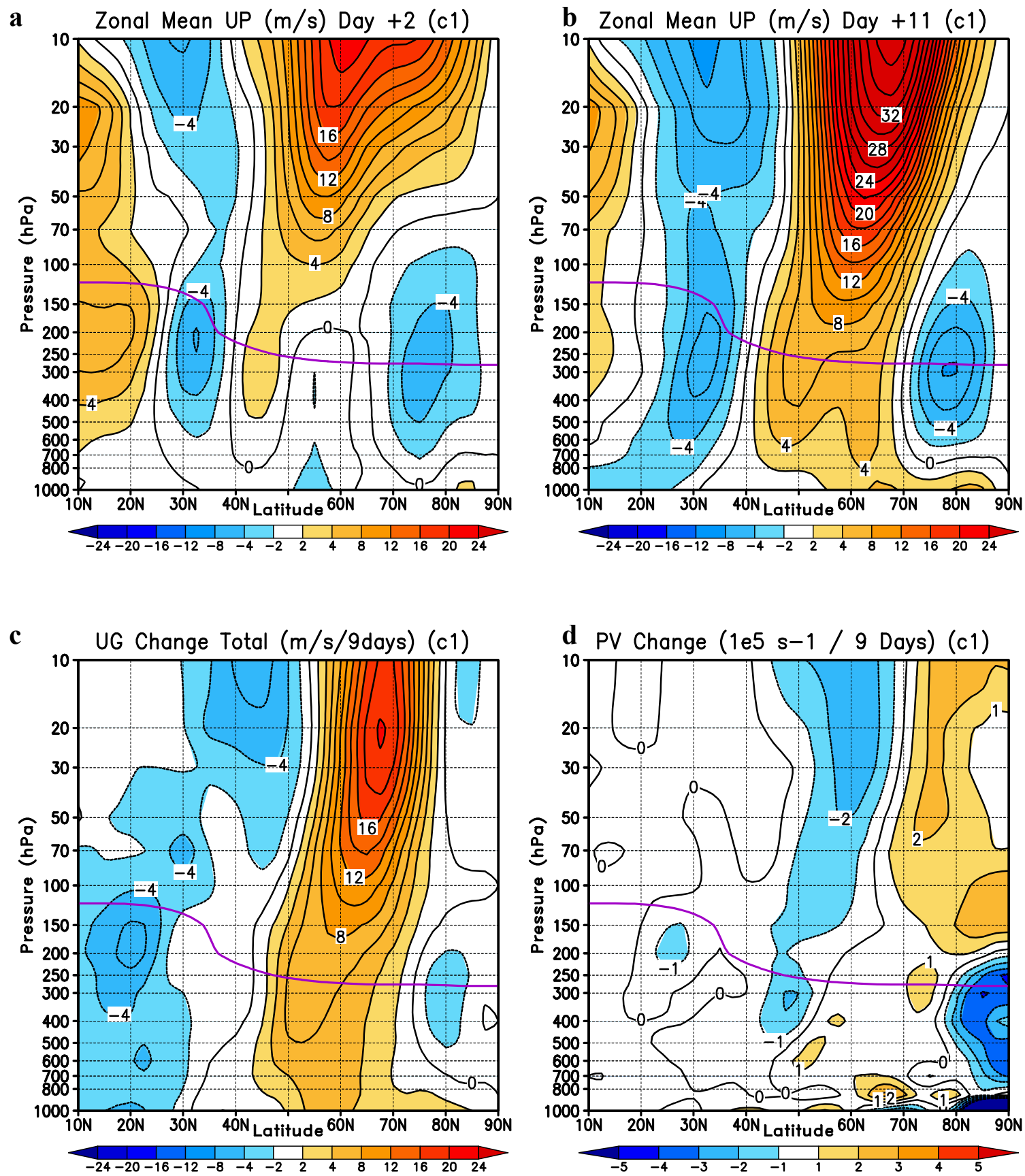

Figure 4.3 Latitude-pressure cross sections of zonal mean fields during case 1 (C1) development. (a) Initial and (b) final zonal wind anomalies (UP; $\mathrm{m} / \mathrm{s}$ ). Nine-day changes in (c) geostrophic zonal wind (UG; m/s) and (d) PV $\left(1.0 \times 10^{-5} \mathrm{~s}^{-1}\right)$. 
development. As pointed out in Black (2002), however, to establish a complete mechanistic framework for the NAM development, it is of fundamental importance to establish the proximate source(s) for the initial stratospheric changes. This is addressed here by calculating the anomalous EP fluxes ${ }^{3}$ and wave driving for the 9-day development period (figure 4.4c). This analysis reveals that case 1 development is associated with anomalous downward and southward EP fluxes within the midlatitude stratosphere and anomalous southward EP fluxes near the midlatitude tropopause. The divergence of the EP flux pattern forces anomalous zonal wind accelerations within the high-latitude stratosphere as well as a meridional acceleration dipole in the vicinity of the tropopause. A spectral decomposition of the EP flux results (figure 4.4d) confirms expectations that the stratospheric wave driving pattern is predominantly due to lowfrequency, intra-annual (periods between 10 days and 1 year). Although we do not expect a precise correspondence between figures $4.3 \mathrm{c}$ and $4.4 \mathrm{c}$, it is evident that the anomalous wave driving in the stratosphere is of sufficient magnitude to account for the observed changes in the zonal wind field at these altitudes. Furthermore, there is a good spatial correspondence in the stratosphere between the pattern of positive wave driving anomalies (representing an anomalous northward PV flux) and the latitudinal dipole in the PV tendency field. We conclude that the initial stratospheric changes likely arise from variations in the propagation of tropospheric planetary waves. Thus, the downward stratospheric influence shown in figure 4.4a should be regarded as a feedback process resulting from tropospheric dynamical processes.

The evolution of case 2 (figures 4.2b, 4.5 and 4.6) is characterized by an

\footnotetext{
${ }^{3}$ See footnote 1, chapter 3 for details on the EP flux vector scaling.
} 

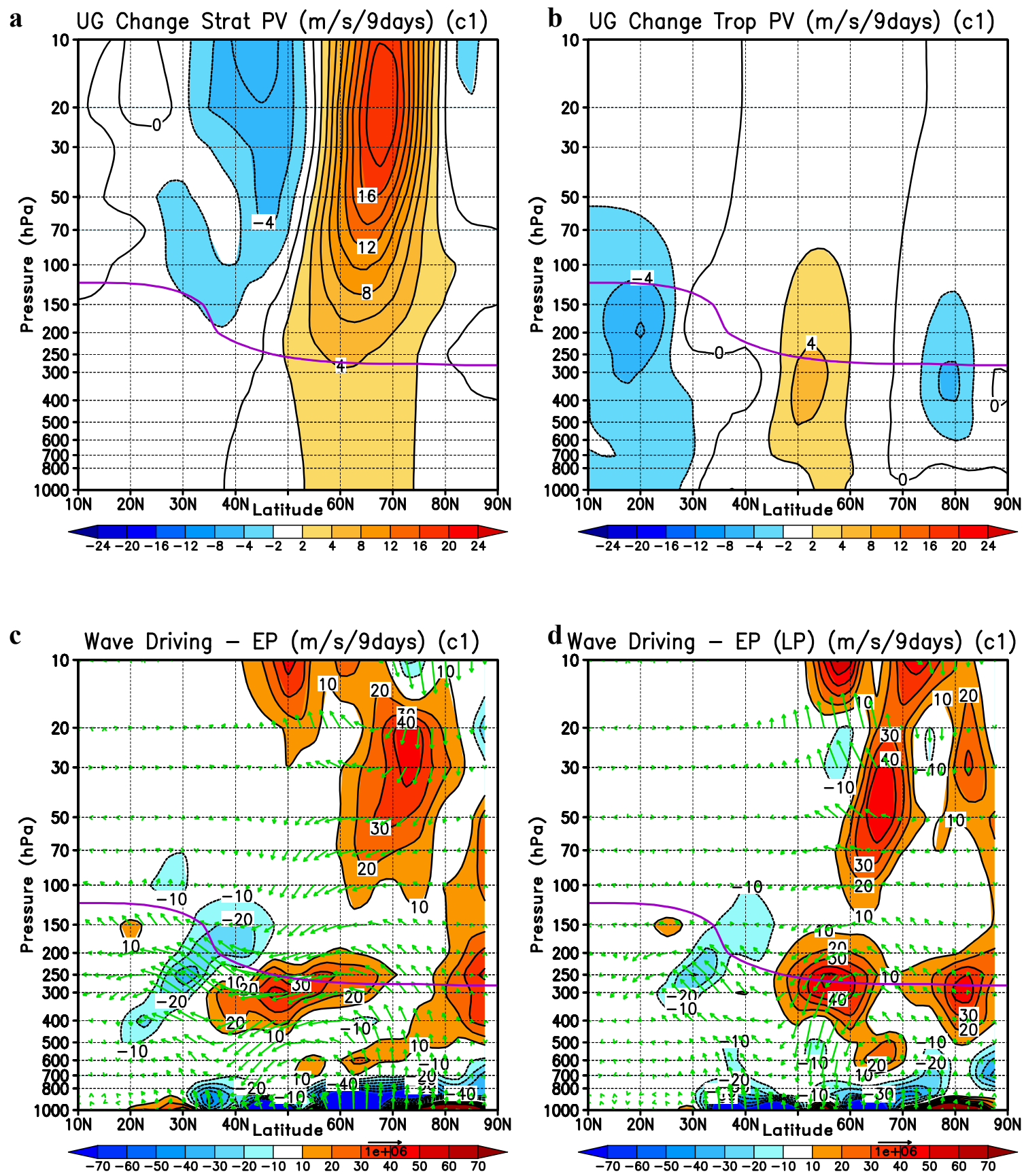

Figure 4.4 Latitude-pressure cross sections of zonal mean diagnostic fields during $\mathrm{C} 1$ development. Nine day UG changes induced by (a) stratospheric and (b) tropospheric PV anomalies $(\mathrm{m} / \mathrm{s})$. (c) Nine-day average anomalies in EP flux (vectors, $\mathrm{m}^{3} \mathrm{~s}^{-2}$ ) and associated wave driving (contours, $\mathrm{m} / \mathrm{s} / 9$ days) and (d) same as (c) but only the forcing due to the low-pass eddy flux anomalies. Scaling of the EP flux vectors same as in figure 3.6. 
analogous (but oppositely signed) zonal wind anomaly development with the highlatitude stratosphere between days +6 and +15 . In this case, however, as the lowerstratosphere development begins, a large amplitude positive zonal wind anomaly feature is present in the high-latitude troposphere and lower stratosphere (figures $4.2 \mathrm{~b}$ and $4.5 \mathrm{a}$ ). Further, the stratospheric zonal wind development is not accompanied by the formation of substantial like-signed zonal wind anomalies within the troposphere (figures $4.2 \mathrm{~b}$ and 4.5b). The initial PV anomaly pattern, consistent with the zonal wind anomaly pattern, is characterized by positive anomalies at high latitudes in the upper troposphere and lower stratosphere and negative anomalies neat the midlatitude tropopause and at upper levels in the high-latitude stratosphere (figure 4.5c). The last feature descends and intensifies over the development period so that by day +15 , negative PV anomalies extend through the depth of the high-latitude stratosphere (figure $4.5 \mathrm{~d}$ ). Substantial positive anomalies remain at high latitudes in the upper troposphere at day +15 , however.

The 9-day zonal wind tendency (figure 4.6a) shows that a tropospheric deceleration does, in fact, occur. As for case 1, the stratospheric PV changes play a strong role in this tropospheric wind tendency (figure 4.6b, this is partly offset by PV changes in the lower troposphere). The end result, however, is that the downward influence is insufficient to overcome preexisting positive zonal wind anomalies at tropospheric levels. Even though the stratosphere successfully acts to decelerate the tropospheric zonal wind field, this ends up simply neutralizing a preexisting tropospheric annular signature of the opposite sign leading to an absence of a corresponding likesigned tropospheric NAM signature. Nonetheless, from the perspective of tropospheric dynamical tendencies, the stratospheric PV changes are important. Lastly, we note that 

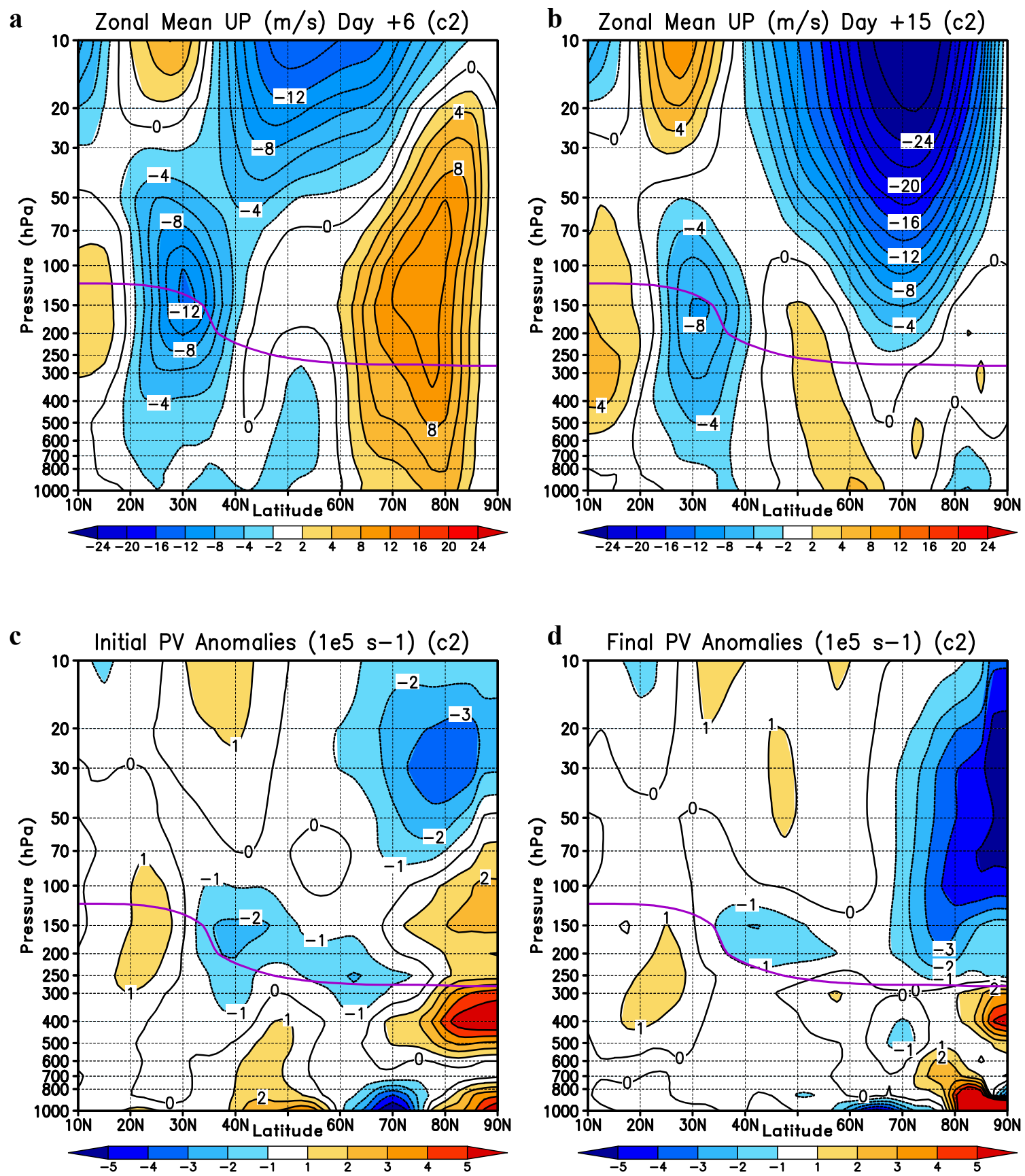

Figure 4.5 Latitude-pressure cross sections of zonal mean fields during case 1 (C1) development. (a) Initial and (b) final zonal wind anomalies (UP; $\mathrm{m} / \mathrm{s}$ ). (c) Initial and (d) final PV anomalies $\left(1.0 \times 10^{-5} \mathrm{~s}^{-1}\right)$. 

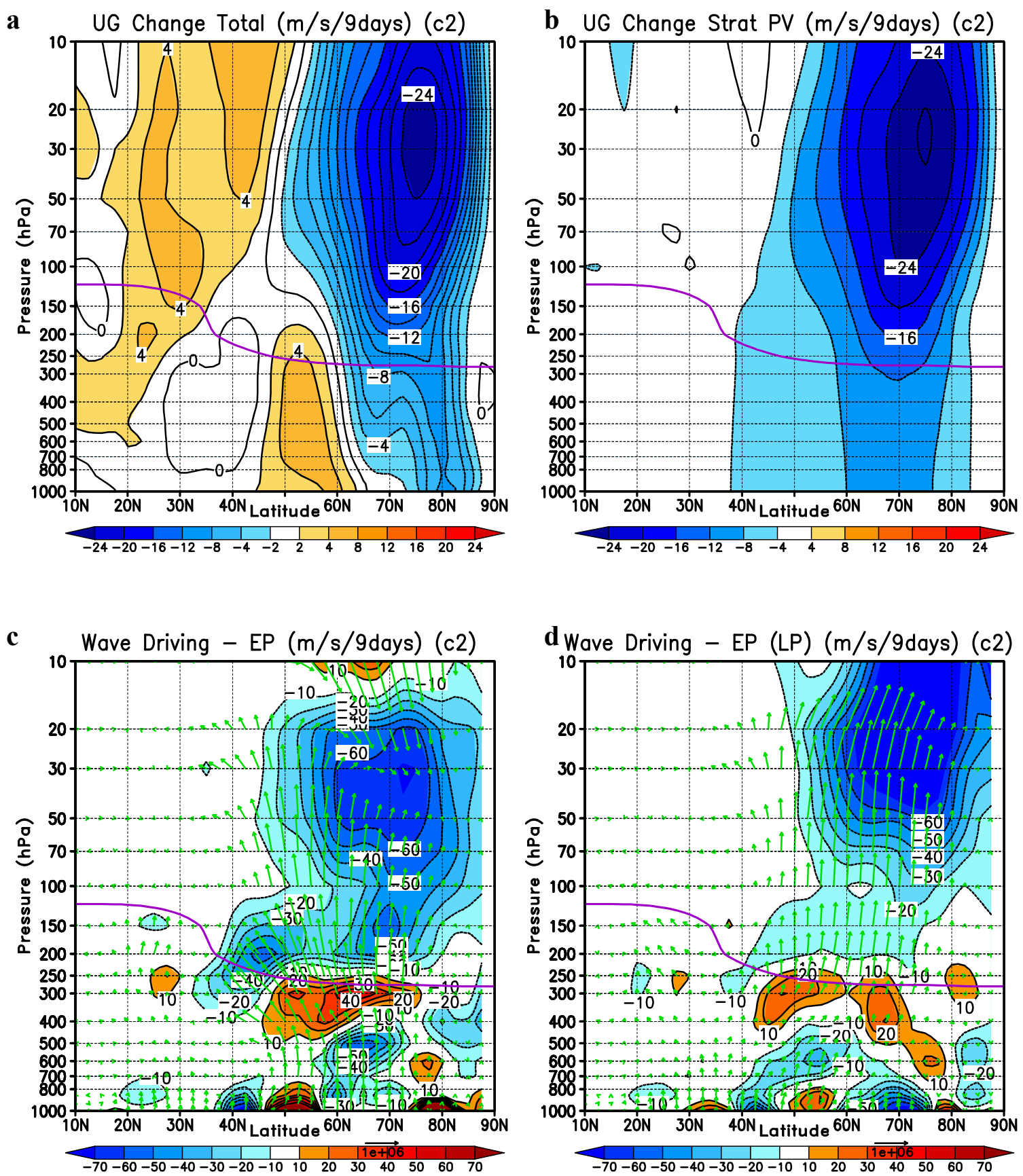

Figure 4.6 Latitude-pressure cross sections of zonal mean diagnostic fields during $\mathrm{Cl}$ development. Nine day changes in (a) total UG $(\mathrm{m} / \mathrm{s})$ and (b) UG induced by stratospheric PV anomalies $(\mathrm{m} / \mathrm{s})$. (c) Nine-day average anomalies in EP flux (vectors, $\mathrm{m}^{3} \mathrm{~s}^{-2}$ ) and associated wave driving (contours, $\mathrm{m} / \mathrm{s} / 9$ days) and (d) same as (c) but only the forcing due to the low-pass eddy flux anomalies. Scaling of the EP flux vectors same as in figure 3.6. 
similar to case 1 , we find evidence that the initial stratospheric circulation changes are largely forced by anomalies (enhancement in this case) in the vertical propagation of tropospheric Rossby waves (figure 4.6c). As in case 1, the anomalous wave driving is mainly due to lowpass eddy fields shown in figure 4.6d.

We next provide a brief overview of the diagnostic results for case 3 . Case 3 represents a negative NAM event that, unlike case 2, is observed to extend into the troposphere. An examination of the evolution of case 3 reveals characteristics that are quite distinct from either case 1 or case 2 . The nature of this behavior is concisely presented in figure 4.7. First we note that the tropospheric NAM development at $60 \mathrm{~N}$ results from a southward latitudinal shift of a well-defined preexisting easterly zonal wind anomaly feature initially centered near $75 \mathrm{~N}$ and extending through the troposphere and lower stratosphere (noting that relatively little latitudinal shift occurred in the middle stratosphere). Second, during this time period, the magnitude of the easterly wind anomalies actually decreased at virtually all altitudes. Third, we note that though the final zonal wind anomaly pattern (figure $4.7 b$ ) very much resembles the canonical NAM pattern, the initial pattern (4.7a) is largely orthogonal to the canonical structure in the troposphere and lower stratosphere (e.g., the initial vertical structure is actually marked by a southward tilt with height). This dissimilarity in the case evolution carries over to the PV inversion results (figures 4.7c,d) that illustrate that the tropospheric (and lower stratospheric) wind changes are primarily controlled by tropospheric PV dynamics. In summary, the case 3 NAM event appears to emerge from the evolving residual signature of a strong preexisting annular mode event having an initial spatial structure very different from the canonical NAM. Although case 3 represents an outlier among the 

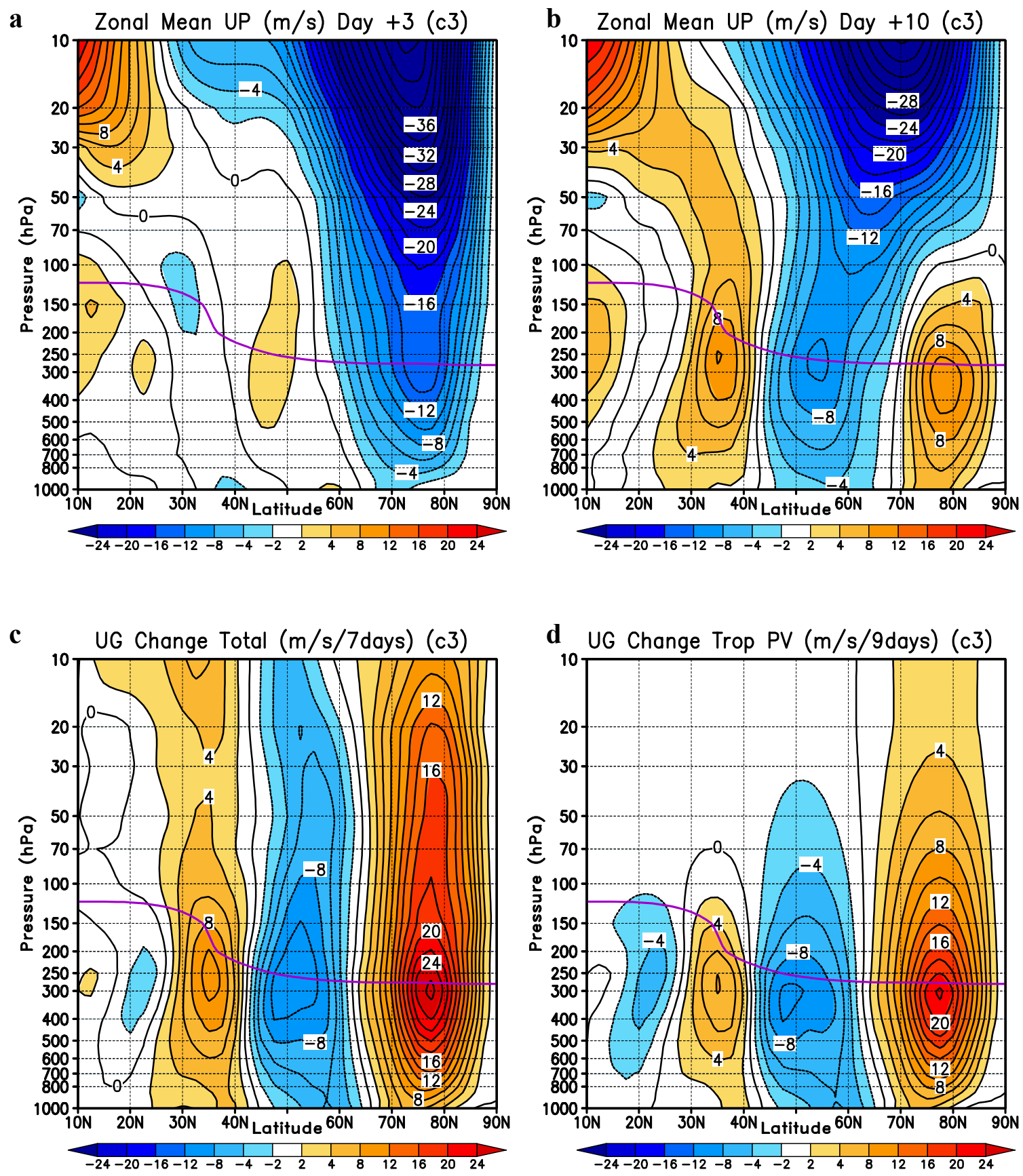

Figure 4.7 Latitude-pressure cross sections of zonal mean fields during case 3 (C3) development. (a) Initial and (b) final zonal wind anomalies (UP; $\mathrm{m} / \mathrm{s}$ ). Nine-day changes in (c) geostrophic zonal wind (UG; $\mathrm{m} / \mathrm{s}$ ) and (d) UG induced by tropospheric $\mathrm{PV}$ anomalies $(\mathrm{m} / \mathrm{s})$. 
cases studied here, the results nonetheless suggest that tropospheric NAM events can be excited in different ways.

\subsection{Nonpropagating NAM Composites}

We next build upon the case studies with supplementary composite analyses. Not surprisingly, the collective synoptic and dynamical evolution of the propagating NAM events generally parallels the top-down canonical evolution described in chapter 3 and elsewhere (Baldwin and Dunkerton, 1999, 2001, Black 2002, Limpasuvan et al. 2004) and, for brevity, is only summarized here. The evolution consists of a cycle initiated by anomalous tropospheric Rossby wave activity into the stratosphere leading to abrupt changes in the zonal mean stratospheric circulation. These changes are then followed by parallel changes in the tropospheric circulation. There is evidence that the stratosphere provides both direct and indirect influence to the tropospheric circulation during the later stages (Black 2002, Limpasuvan et al. 2004, Reichler et al. 2005). The salient question that we attempt to address is why such stratospheric influences do not appear to operate in a sizeable fraction of robust stratospheric events.

Composite evolutionary analyses for the six negative nonpropagating (NN) NAM events and seven positive nonpropagating (PN) NAM events are presented in figures 4.8 and 4.9 respectively. The composite evolutions are constructed with respect to the time at which the $100 \mathrm{mb}$ NAM index attains its peak magnitude, which is referred to as day +15 . The composite time evolutions of the zonal-mean zonal winds anomalies at $60 \mathrm{~N}$ (figures 4.8a and 4.9a) indicate that, for both the NN and PN events, the strongest lower- 
stratospheric circulation intensification occurs during the 10-day period from day +5 to day +15 . As for case 2 , in both composites the lower-stratospheric development is not accompanied by the formation of significant corresponding zonal wind anomalies within the troposphere. (As expected for these nonpropagating composites.) It is interesting to note, however, that both composites exhibit the initial presence at day +5 of oppositely signed zonal wind anomalies in the mid- to upper- troposphere at $60 \mathrm{~N}$ (figures $4.8 \mathrm{~b}$ and 4.9b). The 10-day stratospheric development period begins with an oppositely signed zonal wind anomaly feature located within the troposphere at $60 \mathrm{~N}$ (the approximate central latitude for the canonical manifestation of the tropospheric NAM).

It is evident from figures $4.8 \mathrm{a}$ and $4.9 \mathrm{~b}$ that important zonal wind changes do, in fact, take place at tropospheric altitudes. However, these changes represent an effective elimination of the preexisting tropospheric wind anomaly patterns. To assess the role of stratospheric PV anomalies in enacting the associated tropospheric zonal wind tendencies, we perform piecewise PV inversions for the 10-day development period. The results (shown in 4.8c and 4.9c) show that important stratospheric and tropospheric zonal wind changes are brought about by the redistribution of stratospheric PV. However, at tropospheric altitudes near $60 \mathrm{~N}$, these changes act only to "zero out" the preexisting zonal wind signature. Nonetheless, we again conclude that the stratosphere plays a key role in tropospheric zonal wind tendencies.

Composite EP flux and wave driving analyses are presented for the NN and PN events in figures $4.8 \mathrm{~d}$ and $4.9 \mathrm{~d}$, respectively. These results are consistent with the case studies and canonical prototypes, as we find that the development of negative stratospheric NAM events (NN) is associated with an enhanced upward flux of Rossby 

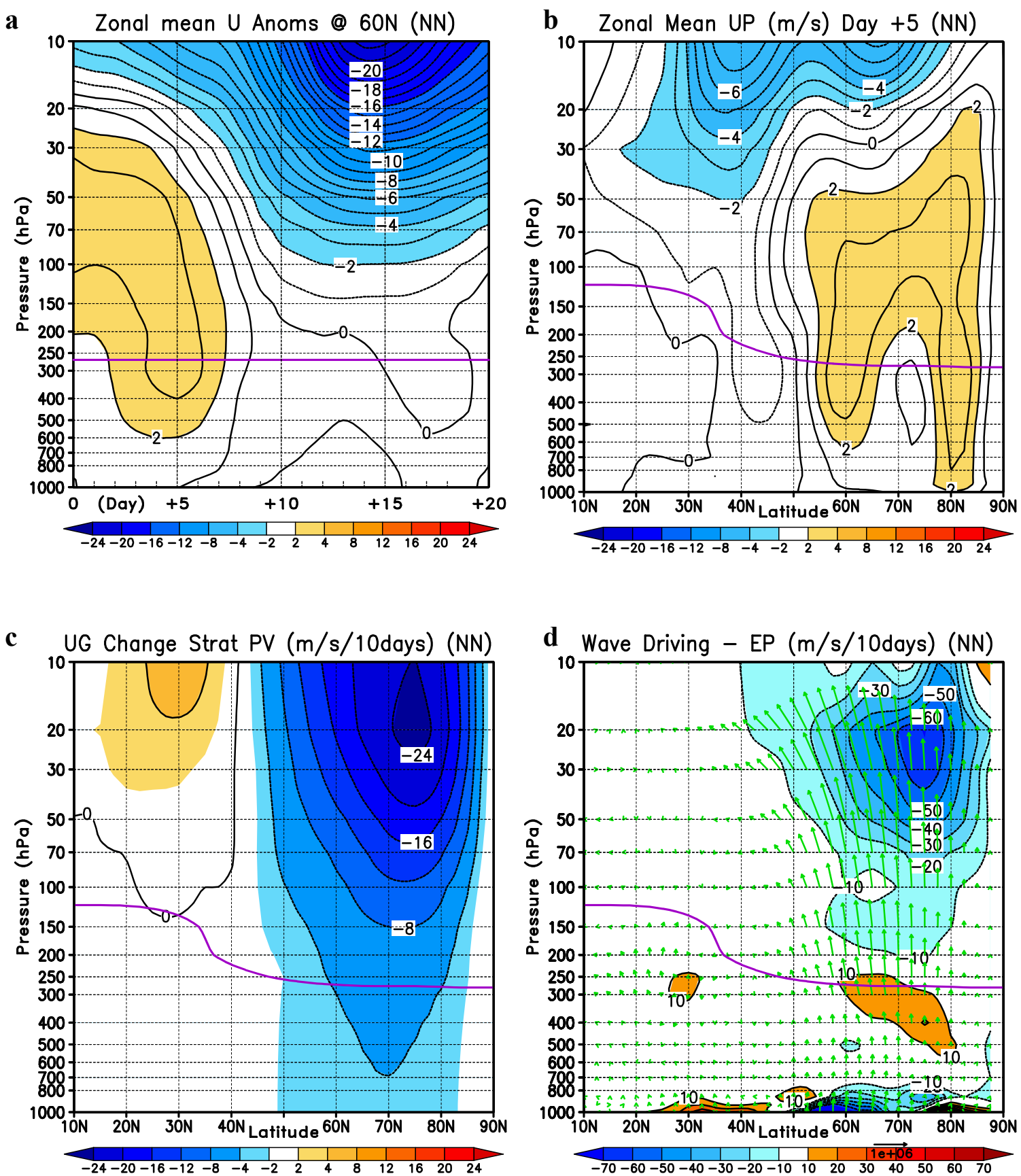

Figure 4.8 Composite analyses of the six NN annular mode events. (a) As in figure 4.2a except for the NN composite development. Latitude-pressure cross sections of composite zonal mean fields during $\mathrm{NN}$ development are presented in the remaining frames. (b) Initial UP $(\mathrm{m} / \mathrm{s})$. (c) Ten-day change in UG induced by stratospheric PV anomalies $(\mathrm{m} / \mathrm{s})$. (d) Ten-day average anomalies in the EP flux (vectors, $\mathrm{m}^{3} \mathrm{~s}^{-2}$ ) and associated wave driving (contours, $\mathrm{m} / \mathrm{s} / 9$ days). 

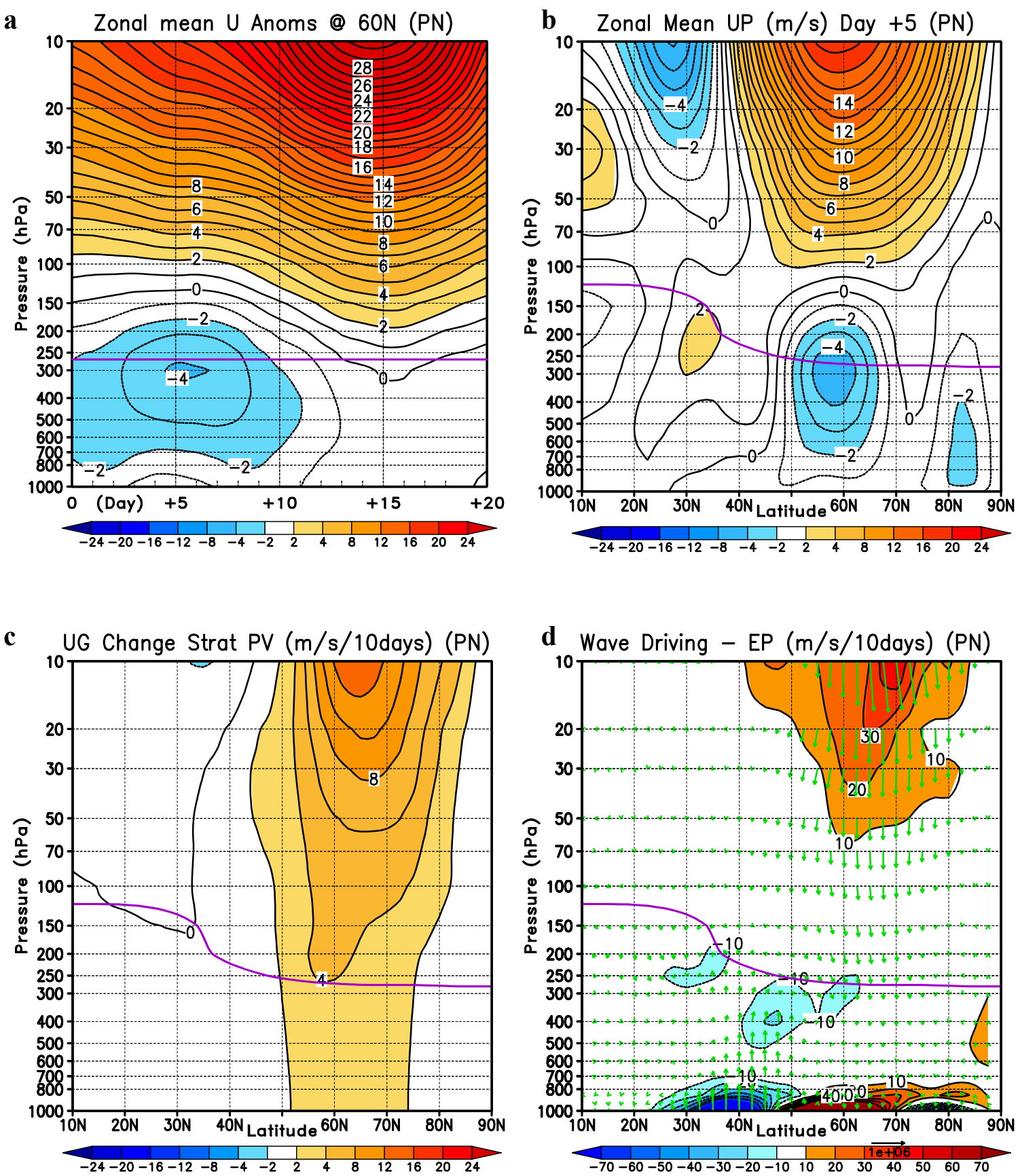

Figure 4.9 As in figure 4.8 but for the composite analyses of the seven PN annular mode events. 
wave activity into the stratosphere. This leads to enhanced wave breaking in the stratosphere (and deceleration of the zonal flow) and southward eddy PV fluxes. Conversely, the PN cases are characterized by positive wave-driving anomalies associated with an acceleration of the polar vortex and northward eddy PV fluxes. It is important to note that the downward anomalous EP fluxes propagating from the stratosphere into the troposphere in the PN composite do not indicate wave activity propagating from the stratosphere downward but rather indicate a weakness in the upwardly propagating tropospheric wave activity.

The composite diagnostic results provide strong support for the idea that nonpropagating NAM events remain confined to stratospheric altitudes because of the initial existence of oppositely signed annular modes at tropospheric altitudes. Finally, we note the striking symmetry that exists between the NN and PN composites, consistent with a linear dynamical behavior.

\subsection{Summary}

The case studies presented have focused on robust stratospheric NAM events with and without succeeding tropospheric NAM manifestations. Those that develop a tropospheric NAM signal (propagating cases) are characterized by robust tropospheric zonal wind anomalies while the nonpropagating cases lack a substantial tropospheric NAM signature. However, examining the zonal wind tendencies reveals that both propagating and nonpropagating cases experience substantial changes in the tropospheric zonal wind field induced by stratospheric PV tendencies. The lack of a tropospheric 
NAM signal in the nonpropagating case is due to a pre-existing, oppositely signed tropospheric zonal wind anomaly. This anomaly effectively blocks the NAM signal from descending into the troposphere as the zonal wind acceleration that would have produced the tropospheric NAM signature instead acts to neutralize the pre-existing anomaly. 


\section{CHAPTER 5}

\section{DYNAMICAL COMPOSITES}

\subsection{Methodology and Composite Statistics}

As overviewed in section 2.4 , to identify periods of intensification/weakening during robust NAM events we have performed composite analyses based jointly on the NAM index time series as well as its time rate of change (hereafter referred to as the index slope). Events were categorized based on the magnitude and sign of the index as well as the index slope. This allows one to construct three-dimensional depictions of the atmosphere during times when the atmosphere is changing within a respective NAM phase. We focus our analysis on times when the NAM signal is strong and quickly changing. In the terminology defined in chapter 2 , we confine the results shown here to the maturing and declining stages of both the positive and negative NAM phases. Reexamining figure 3.1, we see that we are studying the periods leading up to and resulting after the NAM signal has peaked (in contrast to the results presented in chapter 3 which presented results for when the signal was at a maximum at each vertical level).

Here we present composite diagnostic results based on the $150 \mathrm{mb}$ index which effectively provides synthesized information on the joint tropospheric and stratospheric development. Events are considered to be independent if they are separated by 10 days or more. The composite of the maturing (declining) positive NAM events is constructed from 47 (48) time samples derived from 37 (39) independent cases. For negative NAM events, the maturing (declining) composite comes from 73 (42) samples taken from 59 
(35) independent cases.

\subsection{Positive Phase of the NAM}

We first examine the positive phase of the NAM. Figure 5.1 shows the zonallyaveraged zonal wind anomalies and zonally-averaged zonal wind tendencies for the maturing and declining stages of the positive phase of the NAM. The canonical structure of the NAM (e.g., figure 3.1) is apparent in both the maturing and declining zonal wind anomaly fields with weak easterlies at low latitudes and stronger westerlies at mid- to high-latitudes, the latter of which (a) increase in amplitude into the stratosphere and (b) tilts northward with increasing altitude. In both cases, statistically significant wind anomalies are observed to extend through the troposphere reaching all the way to the surface. One notable difference in the zonal wind field between the maturing and declining stage in the apparent "horseshoe" shaped pattern found in the troposphere in figure 5.1c. The associated weakening of surface wind anomalies near $65 \mathrm{~N}$ may prove important and will be discussed later in the chapter. The zonal wind tendency fields also project upon the canonical NAM zonal-wind pattern at mid to high latitudes providing local enhancements (figure 5.1b) or weakenings (figure 5.1d) of the composite NAM event.

Recent work on NAM case studies (Black and McDaniel 2004 and presented in chapter 4) illustrates that a dynamical analysis of the sources for zonal-wind tendencies is critical in obtaining a complete understanding of the short-timescale behavior of the NAM. Comparing figures $5.1 \mathrm{~b}$ and $5.1 \mathrm{~d}$ we observe a clear sign reversal in the wind 

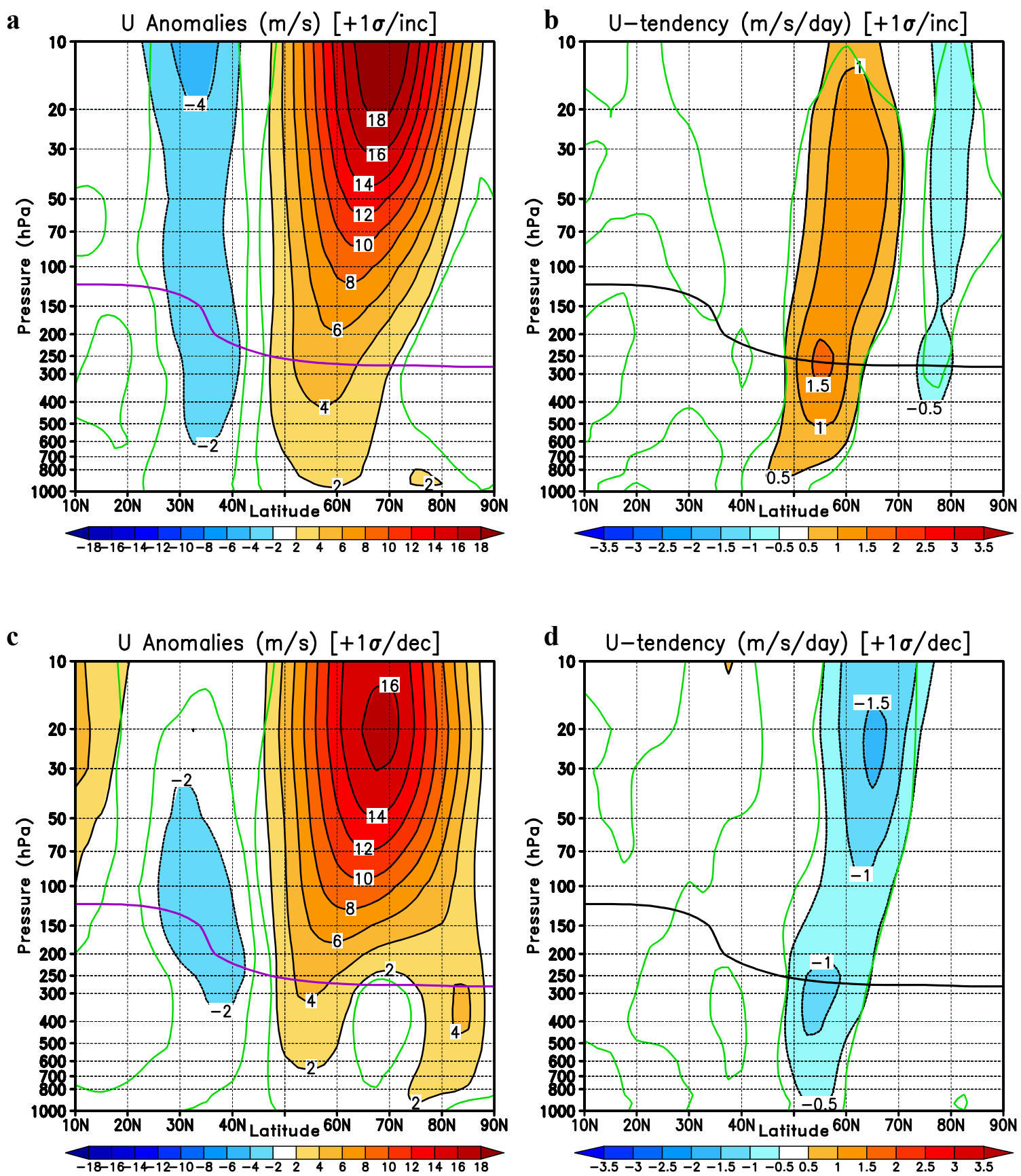

Figure 5.1 Latitude-pressure cross sections of the zonal wind anomaly $[\mathrm{m} / \mathrm{s}]$ and the zonal wind tendency $[\mathrm{m} / \mathrm{s} / \mathrm{day}]$. The green contour represents the $95 \%$ significance level. Figures (a) and (b) show the wind anomalies and tendencies for the maturing stage of the positive phase of the NAM while figures (c) and (d) show the wind anomalies and tendencies for the declining stage of the positive phase of the NAM. 
tendency field, switching from positive during the maturing stage to negative for the declining stage as the stratospheric and tropospheric jet streams simultaneously decelerate back toward climatological conditions. Deducing the factors that lead to this reversal is a key component in understanding and predicting the NAM. It is important to notice that between corresponding maturing and declining stages there is relatively little change in the stratospheric zonal-wind anomaly structure while the zonal-wind tendency completely changes sign.

Figure 5.2 illustrates a quantitative application of the dynamical balance described in equation 2.10 and the time-tendency application of equation 2.19. We note an excellent agreement between the observed zonally-averaged zonal-wind tendency and the sum of the wave driving and Coriolis torque in the free atmosphere (figures 5.2a and $5.2 \mathrm{~b}$, respectively). The primary differences are the relative noisiness and strong positive surface feature observed in the summed dynamical terms (figure 5.2b). The latter feature is likely largely countered by surface drag and thus is not realized in the net wind tendency field (since equation 2.10 neglects friction). Figure 5.2c shows the geostrophic wind tendency calculated by inverting the entire zonal-mean PV-tendency field. The excellent agreement between figures 5.2a and 5.2c illustrates the quantitative utility of the quasigeostrophic framework employed. As in chapter 4, piecewise PV-inversions were performed for subsets of the free atmosphere. Figure 5.2d shows the result of inverting only the stratospheric PV-tendencies for this stage, showing that the stratospheric zonal-wind tendencies are primarily linked to stratospheric PV-tendencies. Of particular interest is the contribution of stratospheric PV-tendencies to the tropospheric zonal-wind tendency field. In this case the stratospheric contribution is 

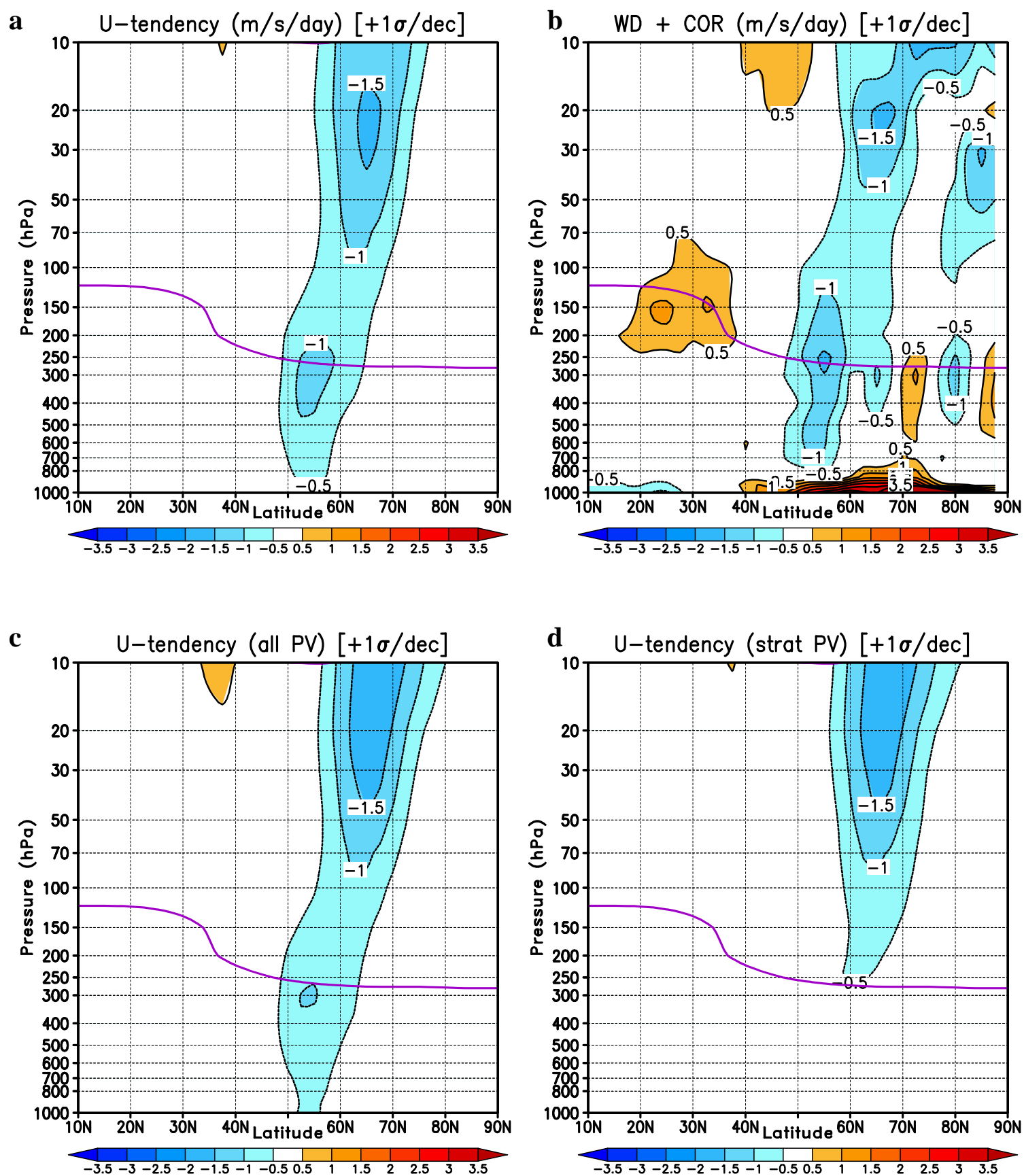

Figure 5.2 Latitude-pressure cross sections for the decline stage of the positive phase of the NAM showing (a) composited zonal wind-tendency [m/s/day], (b) the sum of the wave driving and Coriolis torque $[\mathrm{m} / \mathrm{s} / \mathrm{day}]$, (c) the zonal wind -tendency calculated by inverting the entire domain of the PV-tendency $[\mathrm{m} / \mathrm{s} / \mathrm{day}]$, and (d) the zonal wind-tendency calculated by inverting only the stratospheric PV-tendency. $[\mathrm{m} / \mathrm{s} /$ day $]$. 
small implying that the tropospheric wind tendency is attributable to PV tendencies within the troposphere (tropospheric dynamics).

Figure 5.3 provides a detailed dynamical diagnosis for the maturing stage of the positive phase of the NAM. In this case the zonal wind changes due to stratospheric PV tendencies (figure 5.3a) show an acceleration of the stratospheric polar vortex with a modest contribution extending well into the troposphere (compare to the full tendency field shown in figure 5.1b). Corresponding composite anomalies in the EP-flux ${ }^{1}$ and anomalous wave driving are displayed in figure 5.3b. The anomalous EP-flux pattern is characterized by equatorward and downward fluxes of wave activity at mid-latitudes in both the troposphere and stratosphere. As will be shown latter (figure 5.9), these patterns are primarily forced by large-scale low-frequency eddies. The EP-flux anomalies extending from the stratosphere downward into the troposphere represent an effective reduction in total upward EP-flux. This pattern is thereby consistent with the index of refraction arguments put forth in Hartmann et al. (2000) in which changes in the vertical wind shear alter Rossby wave propagation in the meridional plane. During the positive phase of the NAM, increased vertical wind shear acts to deflect upwardly propagating Rossby wave activity toward the equator, leading to less wave activity reaching the stratosphere at mid- to high- latitudes. Less wave activity leads to weaker wave breaking and therefore less mechanical deceleration of the stratospheric polar vortex. This provides a positive feedback mechanism in which the positive phase of the NAM (a strong, cold vortex) is reinforced. Looking at the wave driving pattern we see a zonalwind acceleration (EP-flux divergence) in the upper troposphere and mid-stratosphere

\footnotetext{
${ }^{1}$ See footnote 1, chapter 3 for a discussion on the scaling of the EP-flux vectors.
} 


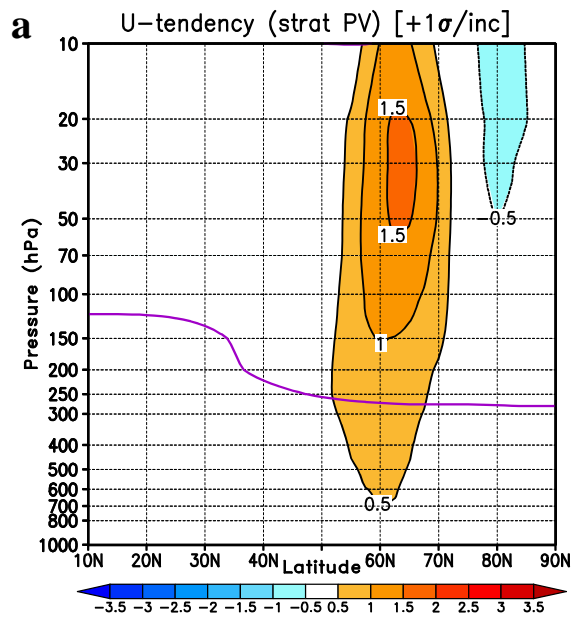

$\mathbf{b}$
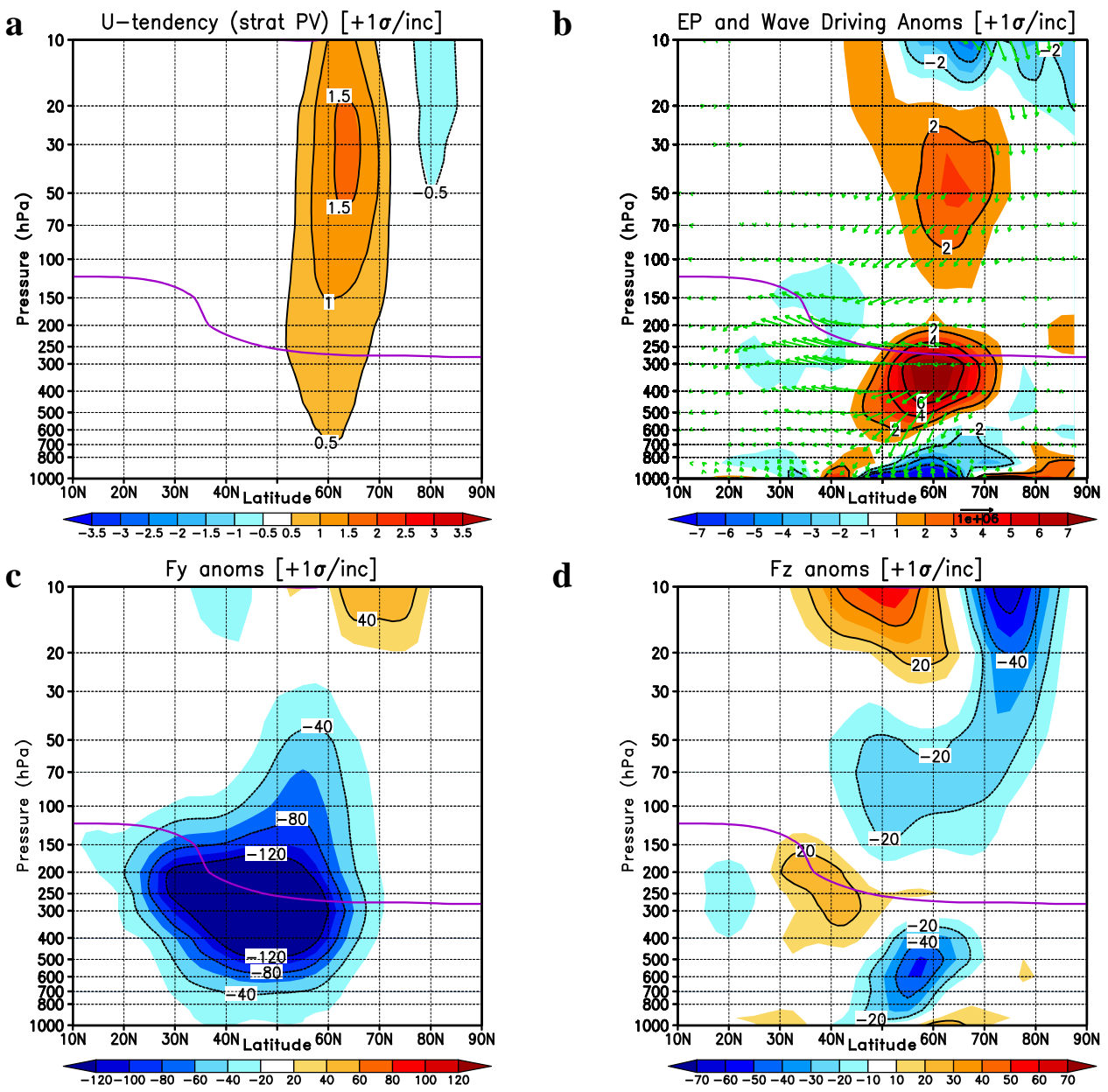

d

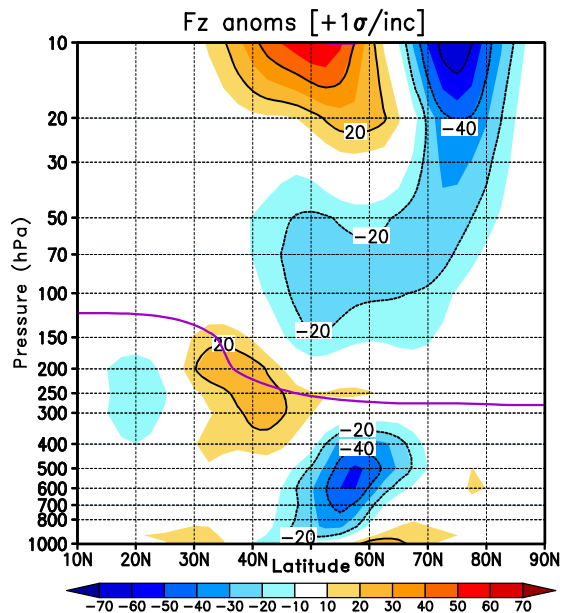

e
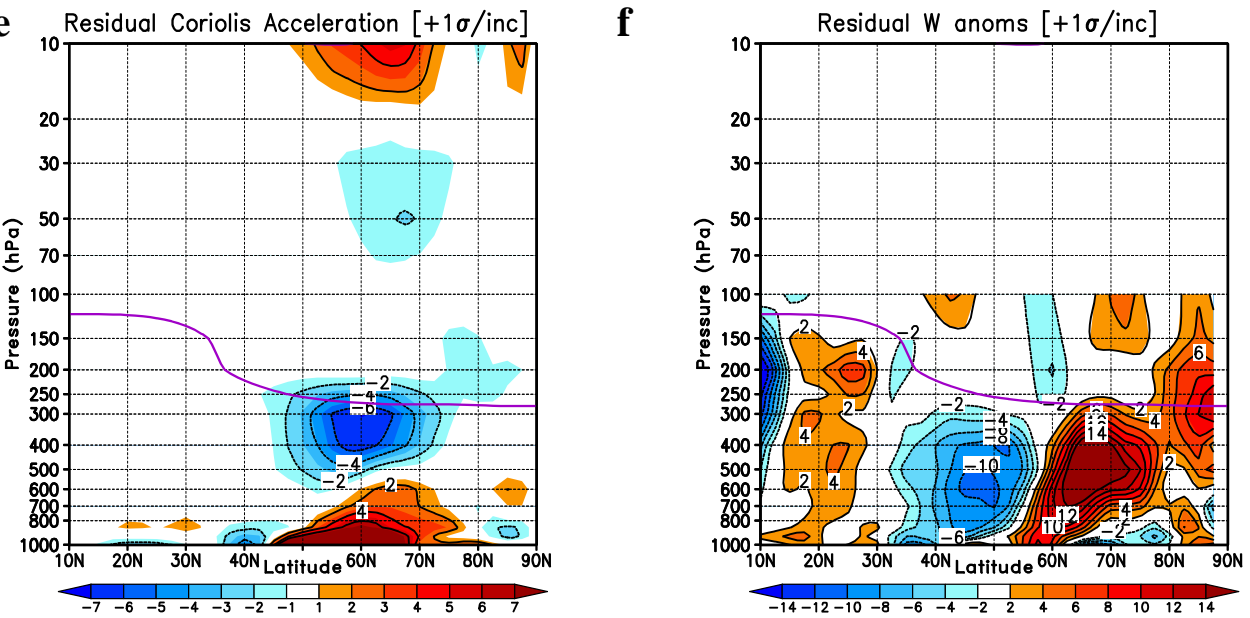

Figure 5.3 Detailed analysis of the mature stage of the positive phase of the NAM. (a) zonal wind tendency due to stratospheric PV-tendencies $[\mathrm{m} / \mathrm{s} / \mathrm{day}]$, (b) anomalous EP-flux vectors $\left[\mathrm{m}^{3} \mathrm{~s}^{-2}\right]$ and associated wave driving [m/s/day] (only EP vectors significant at $95 \%$ are plotted), (c) meridional component of the anomalous EP-flux $\left[10^{6} \mathrm{~m}^{3} \mathrm{~s}^{-2}\right]$, (d) vertical component of the anomalous EP-flux $\left[10^{4} \mathrm{~m}^{3} \mathrm{~s}^{-2}\right]$ (e) the Coriolis torque due to the residual meridional velocity [m/s/day], and (f) the residual vertical velocity $\left[10^{-4} \mathrm{~m} / \mathrm{s}\right]$. EP flux vectors are scaled to point in the proper direction while enhancing magnitudes at successively higher altitudes (see text for details). The local EP flux divergence (which is not preserved in this scaling) can be inferred from the wave driving anomalies. 
that is consistent with the observed zonal-wind tendency. We point out, however, that the structure of this pattern is likely sensitive to details of the tropospheric zonal wind structure (which changes between maturing and declining stages). The meridional and vertical components of the EP-flux are plotted separately in figures 5.3c and 5.3d, respectively. We note that the individual flux components shown in these figures (and later in figures 5.4, 5.6, and 5.7) are scaled in the same manner as the EP-flux vectors displayed throughout.

As discussed in chapter 4 , the wave driving field is directly proportional to the meridional eddy flux of potential vorticity. As such, the positive wave driving anomaly observed at stratospheric altitudes corresponds to an anomalous northward flux of PV, providing PV increases (decreases) to the north (south) of $65 \mathrm{~N}$. This is precisely the type of stratospheric PV redistribution that is necessary to induce the tropospheric westerly anomalies observed in figure 5.3a. Consequently, we regard the direct downward stratospheric influence diagnosed in figure 5.3a as a stratospheric feedback mechanism with roots in the tropospheric wave field (as in chapter 4). However, it is evident from figure 5.3 that this downward stratospheric influence is considerably weaker in magnitude than the co-located positive wave driving observed in the upper troposphere near $60 \mathrm{~N}$.

Figures 5.3e and 5.3f show the respective associated meridional and vertical ${ }^{2}$ residual circulations. A clear secondary circulation is evident in the troposphere that is consistent with the wave driving pattern. In physical terms, the mechanical acceleration

\footnotetext{
${ }^{2}$ The NCEP/NCAR reanalysis only archives vertical winds up to $100 \mathrm{mb}$, thus the residual vertical circulation can only be displayed up to this altitude.
} 
due to the wave driving seen in the upper troposphere leads to a local thermal wind imbalance. A nonlocal adjustment of the underlying temperature field is necessary to restore the balance. This is achieved via induction of a secondary circulation in which adiabatic cooling occurs at high latitudes with corresponding warming at lower latitudes. Near the surface the impact of the Coriolis torque upon the secondary circulation will provide a zonal wind acceleration leading to an effective vertical redistribution of the initially localized zonal wind acceleration due to the wave driving.

To better understand the evolving dynamics of positive NAM events, we perform the same diagnostic analyses but for the declining stage (figure 5.4). Looking at figures 5.1c and 5.1d we see that during this stage the polar vortex remains anomalously strong but the zonal-wind tendency changes sign, with zonal-wind decelerations comparable in magnitude to the maturing stage accelerations. As illustrated in figure 5.2, the stratospheric component of the wind-tendency is recovered by inverting the stratospheric PV-tendency field (figure 5.4a) while in this case tropospheric tendencies are, by inference, attributable almost solely to tropospheric PV changes. This indicates a weaker role for the direct downward stratospheric influence during the declining stage versus the maturing stage.

In the EP-flux plot (figure 5.4b) we now see enhanced anomalous upward wave propagation in both the troposphere and stratosphere at mid-latitudes. Interestingly, at tropospheric altitudes the enhanced upward flux occurs within the latitudinal minimum in the zonal-wind anomaly field $(\sim 65-70 \mathrm{~N})$ (figure 5.1c). The associated EP-flux convergence acts to slow the zonal flow in both the upper troposphere and stratosphere as evident in the wave driving (figure 5.4b). An important result arises in comparing figures 

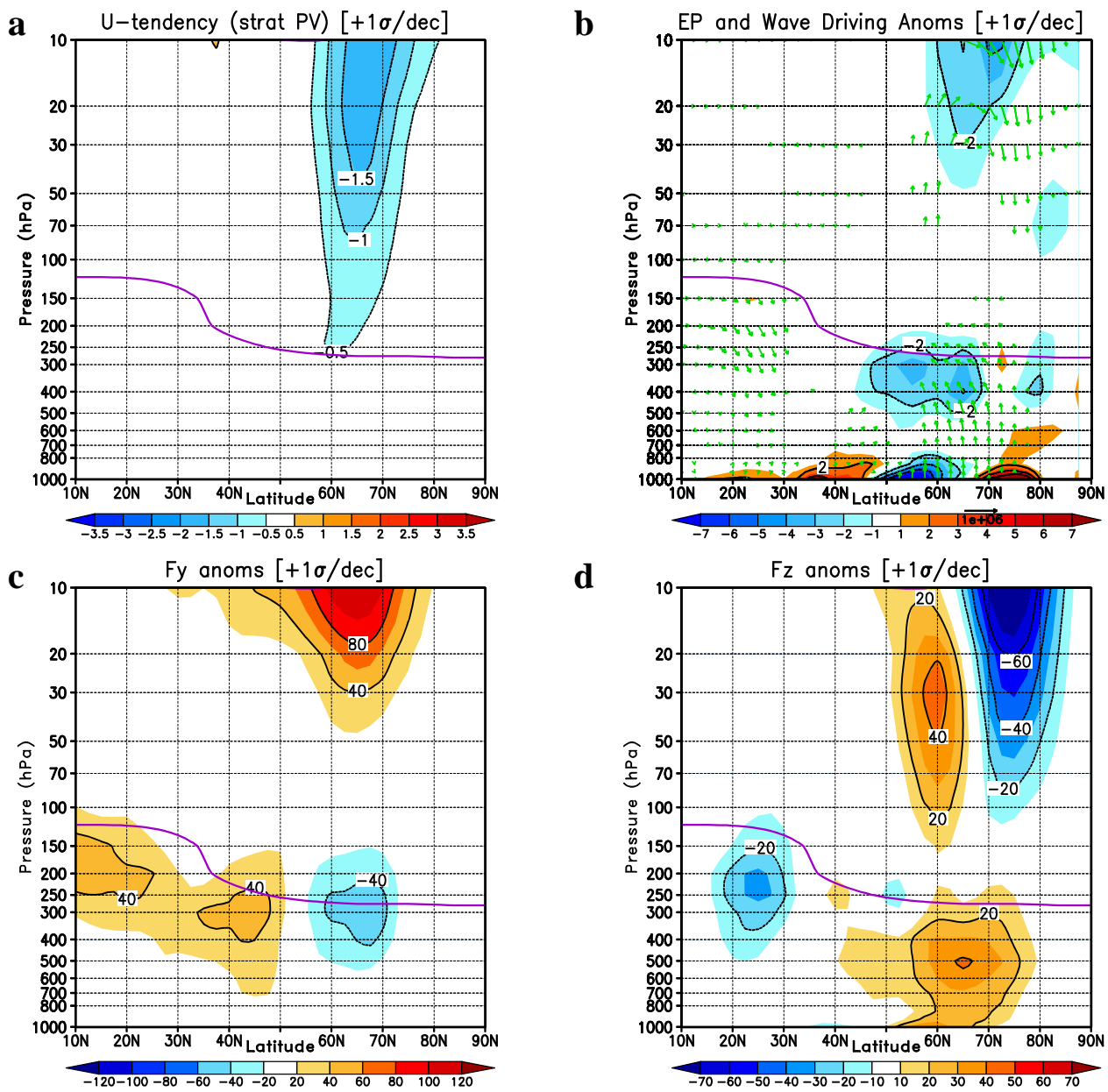

d

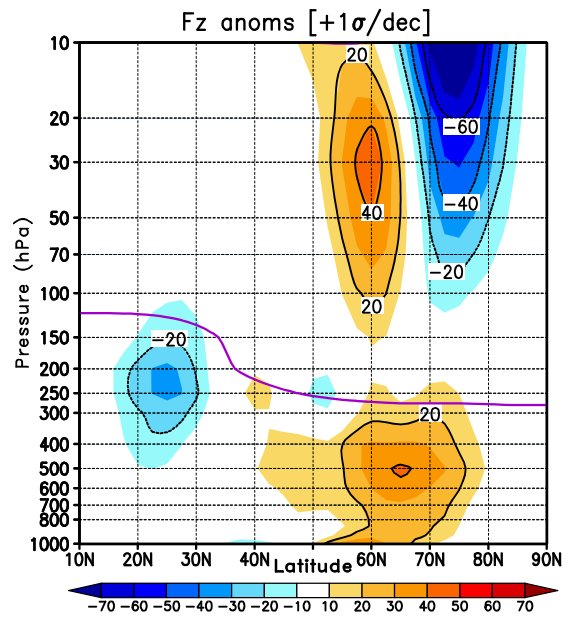

e Residual Coriolis Acceleration $[+1 \sigma / \mathrm{dec}]$
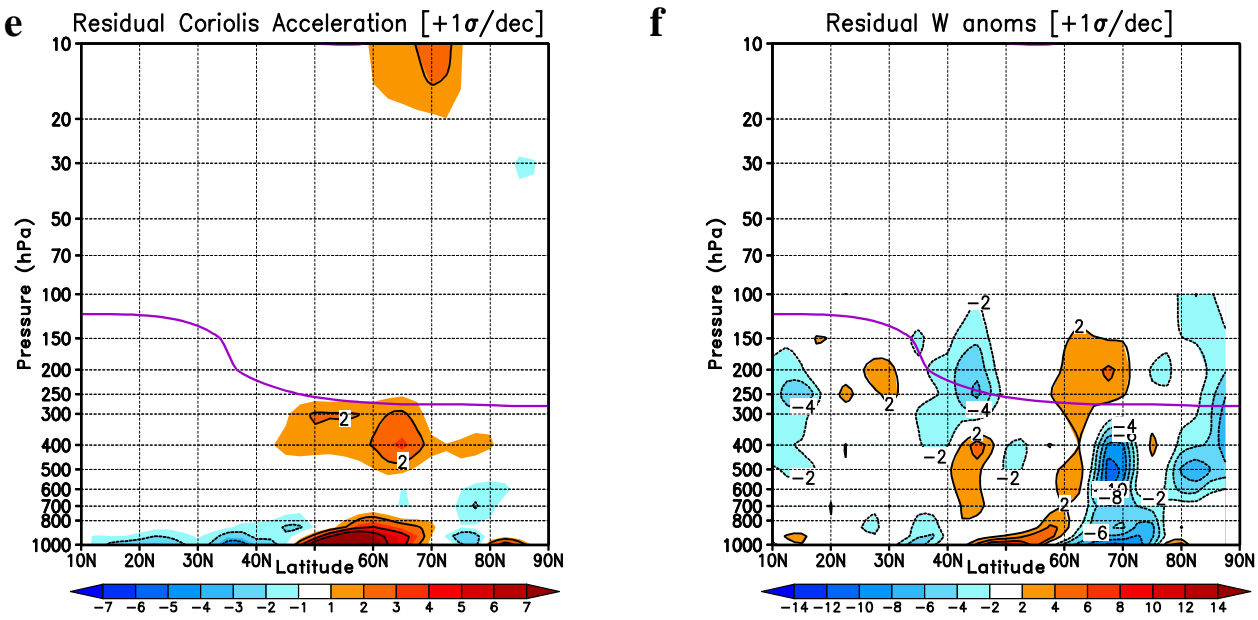

Figure 5.4 As in figure 5.3 but for the decline stage of the positive phase of the NAM. 
$5.3 \mathrm{c}$ and $5.3 \mathrm{~d}$ to figures $5.4 \mathrm{c}$ and $5.4 \mathrm{~d}$. The central features are (a) the change in sign of the anomalous tropospheric heat flux (figures 5.3d, 5.4d) and (b) the effective disappearance of the strong southward (northward) EP (zonal momentum) flux observed during the maturing stage (figure 5.3c). If, in the declining stage, we were to assume that the vortex is simply radiatively relaxing back to climatological conditions, we would expect the anomalous heat and momentum fluxes to be small. However, this is not the case as robust northward heat flux anomalies (upward EP flux anomalies) are observed during the declining stage. The reversal of sign in the heat flux is also accompanied by a northward shift in the latitudinal location of the total heat flux maximum. Figures $5.4 \mathrm{e}$ and $5.4 \mathrm{f}$ indicate that the residual circulation is weaker and less coherent than for the maturing stage. This is due to relatively weak upper-tropospheric wave driving requiring less Coriolis torque to balance it.

\subsection{Negative Phase of the NAM}

We next perform parallel diagnoses for the negative phase of the NAM. One goal in examining the dynamics of the negative phase is to assess the similarities and differences that exist between positive and negative NAM events. It is also of interest to contrast our results with the recent observational study of sudden stratospheric warmings (SSW) events by Limpasuvan et al (2004). Figure 5.5 displays the zonal-wind anomalies and the zonal-wind tendency for the maturing and declining stages of negative NAM events. Both stages are characterized by a weakened stratospheric polar vortex with significant easterly anomalies extending down to the surface. As for positive phase 

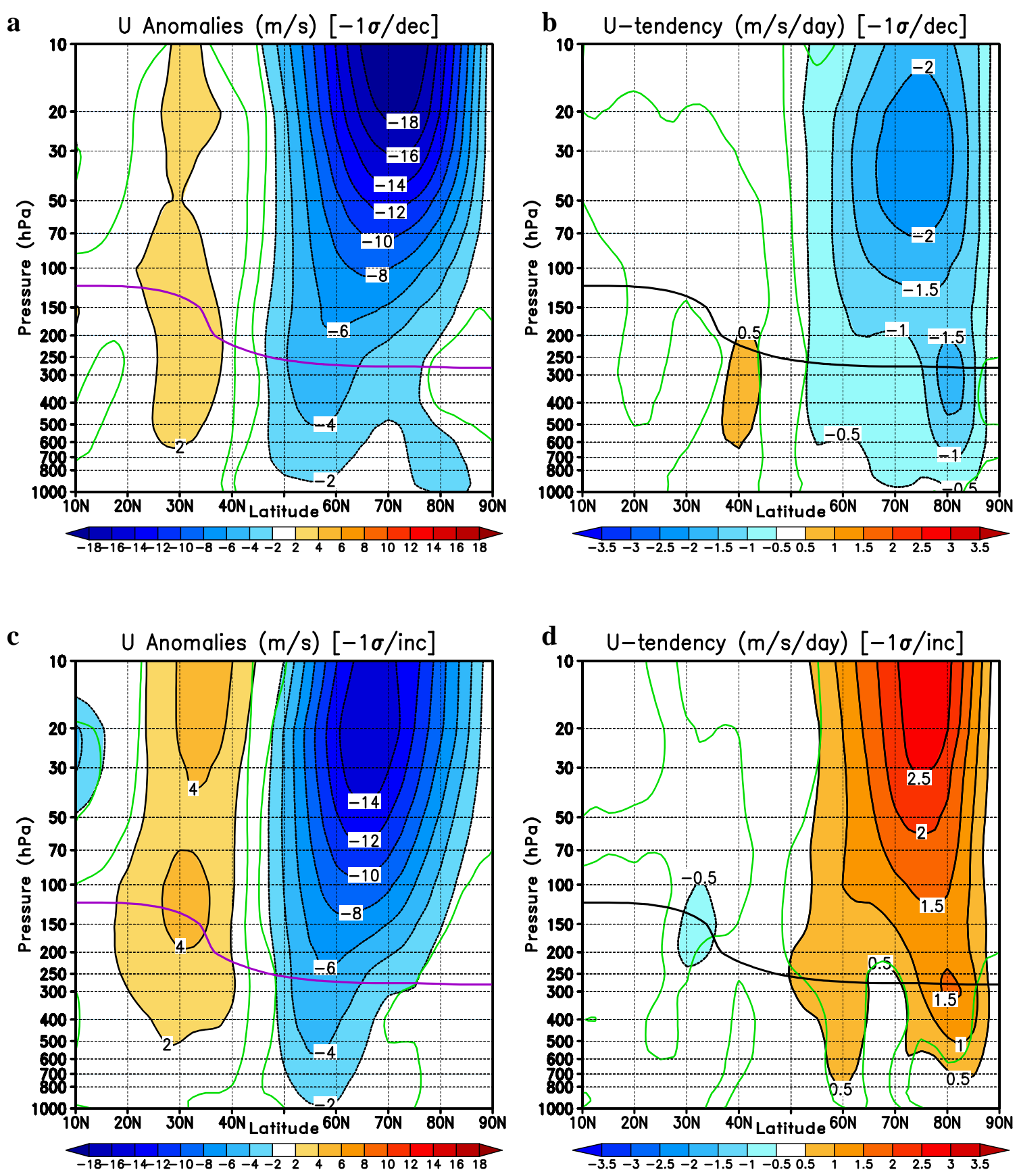

Figure 5.5 As in figure 5.1 but for the negative phase of the NAM. 
events, both maturing and declining stages exhibit the canonical NAM structure of an extratropical zonal wind anomaly pattern that increases in strength and tilts northward with height (from a surface minimum located near $55 \mathrm{~N}$ ). We note that this northward tilting anomaly structure is actually absent in the SSW composites of Limpasuvan et al (2004 - Figure 3). The easterly zonal wind anomaly structure they identify in association with SSW events are, in fact, characterized by a southward tilt with respect to height as the strongest easterly anomalies in the mid to lower troposphere are located near $75 \mathrm{~N}$. This difference indicates, at the very least, a lack of one-to-one correspondence between the tropospheric NAM events studied here and the corresponding tropospheric manifestation of SSW events. On the other hand, the maturing stage composite (figure 5.5a) does show a weaker (but still significant) lobe of tropospheric easterly anomalies extending to $80 \mathrm{~N}$, leading to another "horseshoe" shaped anomaly pattern in the tropospheric zonal wind anomaly field. Interestingly, this feature disappears between the maturing and declining stages (the reverse of the positive NAM behavior).

As for the positive NAM events, the wind tendency patterns (figures $5.5 \mathrm{~b}$ and 5.5d) again show a sign reversal as the event transitions from the maturing to declining stages. During the maturing stage the combined effect of the wave driving and Coriolis torque at stratospheric altitudes is to further slow the vortex, leading to a stronger negative NAM signal in the stratosphere (anomalously weak vortex). One difference with the positive phase results is that the tropospheric wind tendency patterns (figures $5.5 \mathrm{~b}$ and $5.5 \mathrm{~d}$ ) are latitudinally phase shifted northward with respect to the wind anomaly patterns (figures 5.5a and 5.5c).

Figure 5.6 provides a detailed dynamical diagnoses of the maturing stage of the 
negative NAM phase. The most notable feature in figure $5.6 \mathrm{~b}$ is the large upward EPflux extending into the stratospheric polar vortex, leading to a substantial zonal deceleration in the stratosphere and upper troposphere. The EP-fluxes for this stage generally oppose those seen in the maturing stage of the positive NAM (figure 5.3b). In this case, the EP-fluxes near the tropopause are ducted poleward until approximately $55 \mathrm{~N}$ with they become redirected upward into the stratosphere. As for the positive NAM events, these anomaly patterns are primarily due to the effects of large-scale lowfrequency eddies (see figure 5.10).

Examining the individual components of the EP flux (figures 5.6c,d) and comparing to those found in figure 5.3, we find a considerable amount of reverse symmetry between positive and negative NAM events, especially in the troposphere. The main distinction is that the positive stratospheric vertical flux anomaly observed in figure 5.6d has a considerably weaker negative counterpart during positive NAM events (figure 5.3d). A coherent anomalous residual circulation induced by the wave driving is evident in the bottom two panels of figure 5.6. The negative wave driving in the upper troposphere leads to a clockwise (thermally direct) residual circulation during the negative phase, opposite to that noted during the positive phase, and more broad in latitudinal extent.

Figure 5.7 shows the same analyses as figure 5.6 but for the declining stage of the negative NAM. The anomalous wave driving changes sign, mainly due to an effective reversal in the vertical EP-flux pattern (figure 5.7a) which is distinguished by a coherent pattern of downward flux anomalies extending from the mid-stratosphere down to the surface (providing a net reduction in the total upward EP-flux). The other notable aspect 

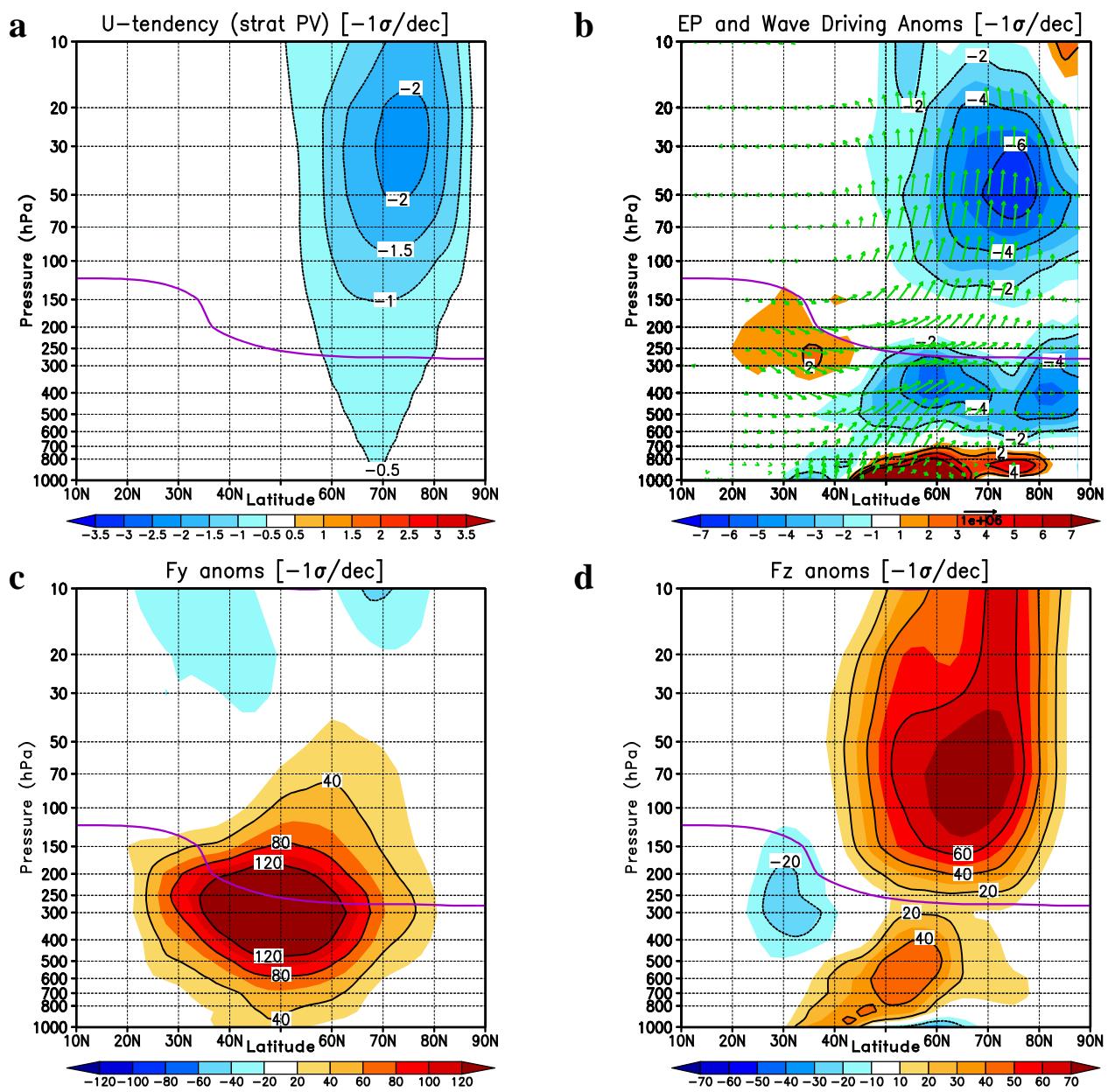

d

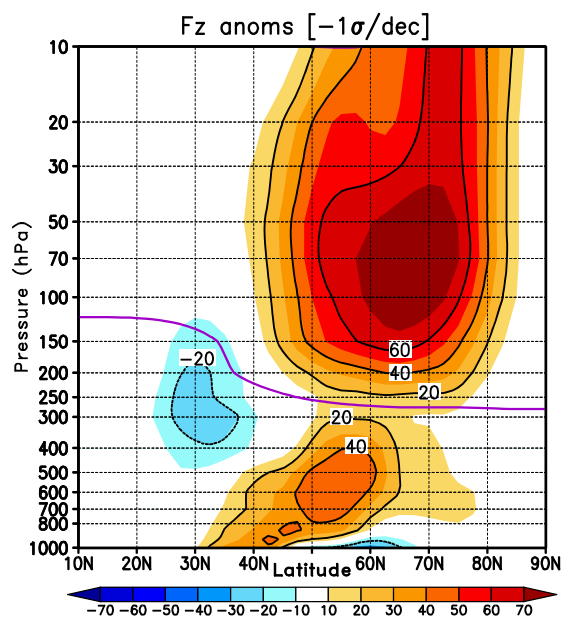

e Residual Coriolis Acceleration $[-1 \sigma / \mathrm{dec}]$
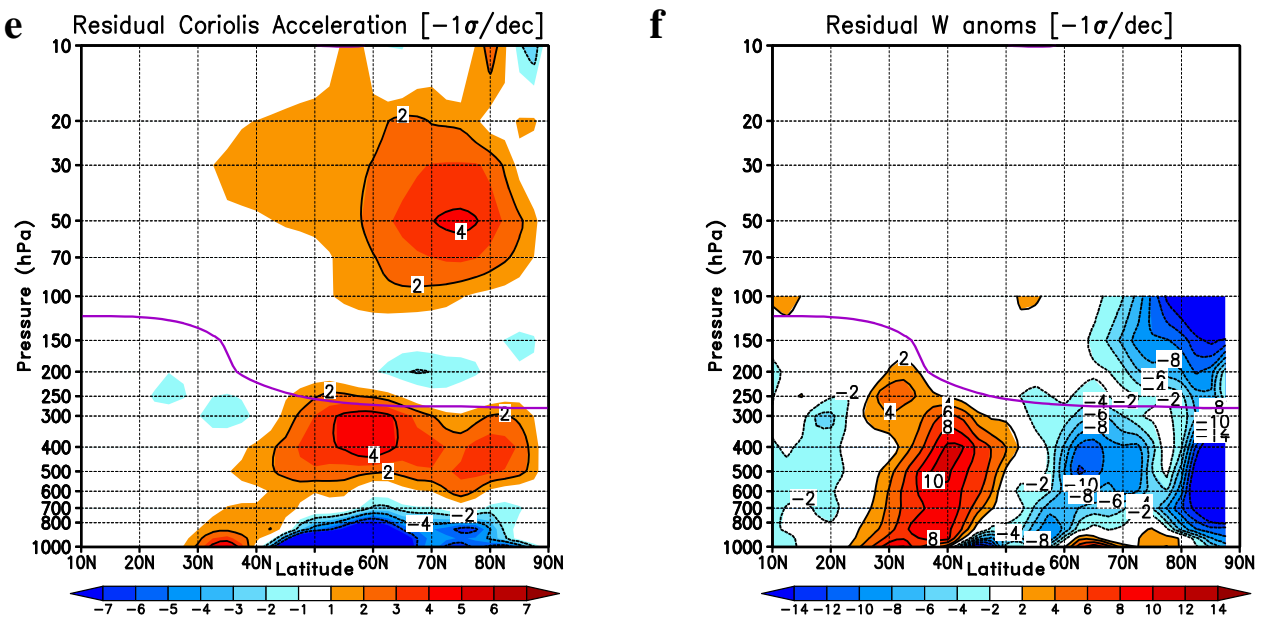

Figure 5.6 As in figure 5.3 but for the mature stage of the negative phase of the NAM. 

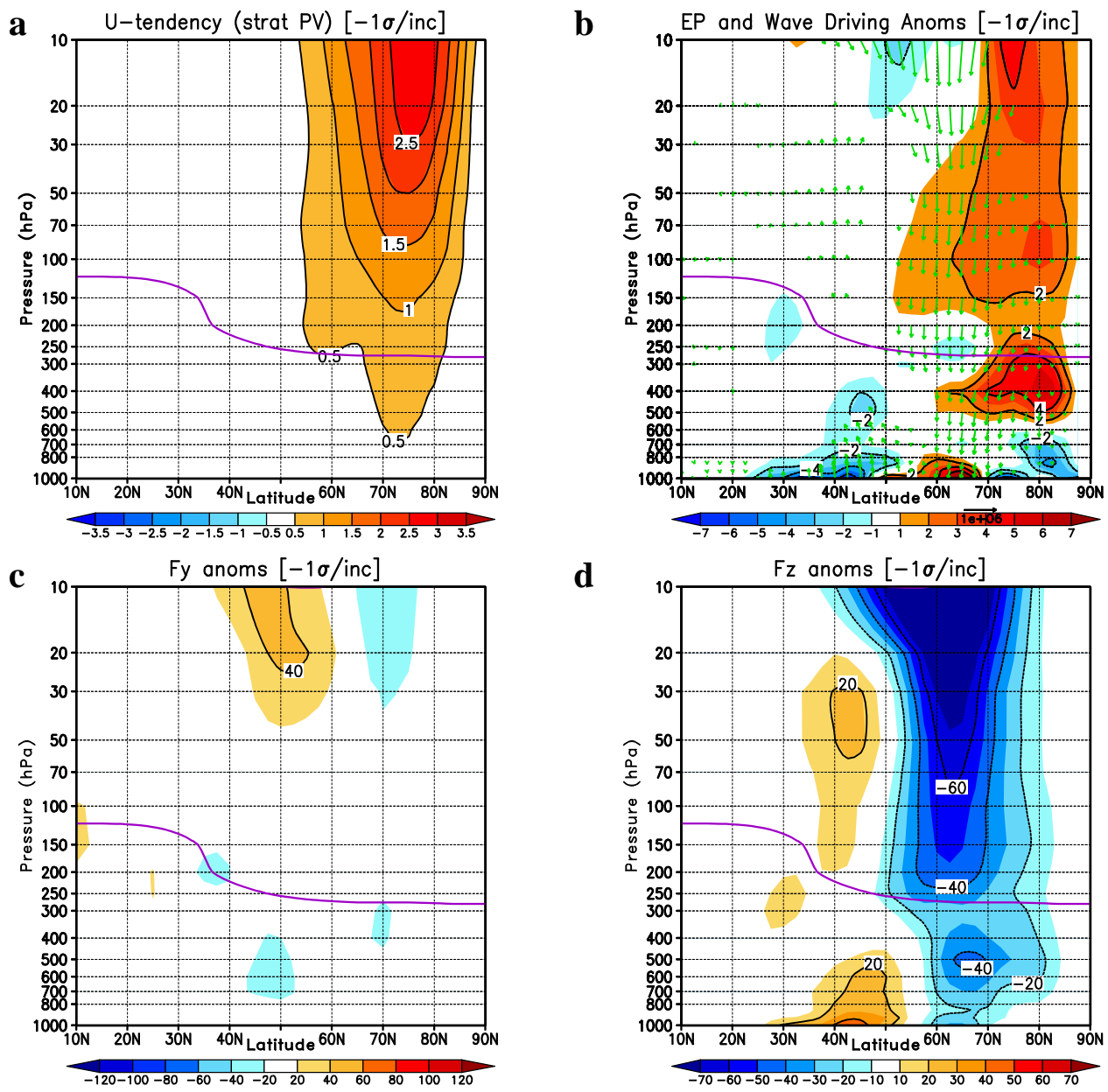

d
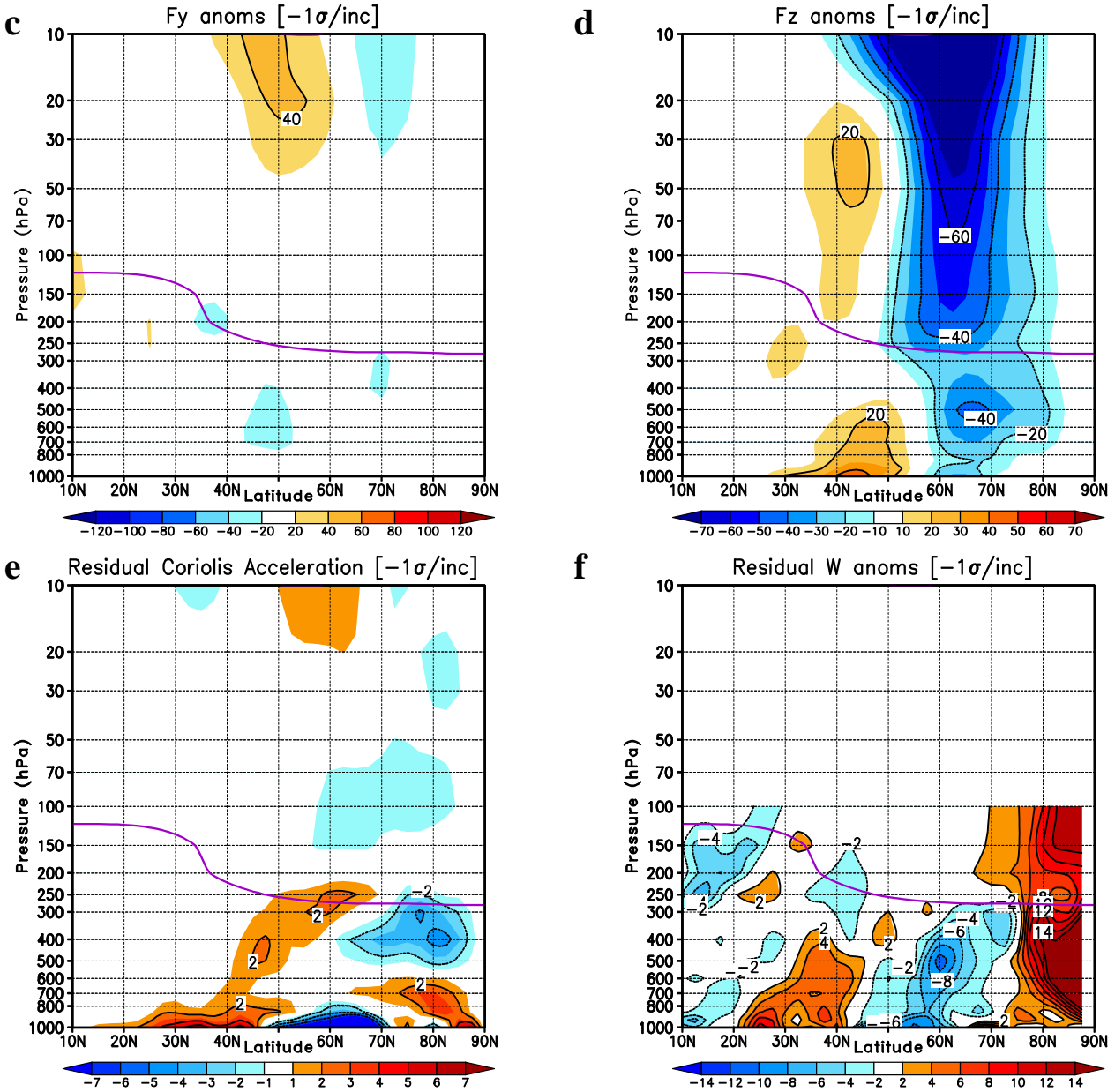

Figure 5.7 As in figure 5.3 but for the decline stage of the negative phase of the NAM. 
of the declining stage is the virtual elimination of the anomalous northward (southward) meridional EP (momentum) flux observed during the maturing stage (figure 5.6c). These aspects parallel the behavior of positive NAM events (but with signs reversed). A secondary feature is a meridional split of the heat flux pattern in the lower troposphere during the transition, yielding a dipole anomaly centered at $55 \mathrm{~N}$ (figure 5.7d). Considering the location of the largest changes in the tropospheric heat fluxes between the maturing and declining stages for positive and negative NAM phases, we note that the region around $\sim 60 \mathrm{~N}$ appears pivotal in producing the stage transition for NAM events.

There are important distinctions between the diagnostic analyses shown in figures 5.6 and 5.7 and parallel diagnoses of SSW performed by Limpasuvan et al (2004). First we note that, although the EP-flux pattern displayed in figure $5.6 \mathrm{~b}$ qualitatively resembles growth phase of Limpasuvan et al. (third column of their figure 3), the associated wave driving signature at high latitudes in the upper troposphere is of opposite sign (negative wave driving in our analyses vs. positive wave driving in their analyses). Furthermore, Limpasuvan et al. continue to observe substantial northward EP-fluxes during event decay while we observe a virtual elimination (figure 5.7c) of the prominent initial northward EP flux signature (figure 5.6c). Additional distinctions will become apparent in the following section. 


\subsection{Regional Wave Forcing and Spectral}

\section{Decomposition}

We next examine the regional wave dynamics associated with the growth and decay of positive and negative NAM events. In pursuing the problem we also implicitly address the questions of the extent to which the eddy fluxes associated with the composite anomaly field are able to account for the zonal mean tendency dynamics (recognizing that anomalous wave forcing due to case-to-case variability may also be significant in the zonal-mean dynamics). To this end we assess the anomalous Plumb flux by considering the net impact of the composite eddy field upon the total Plumb flux field (see chapter 2 for a more complete discussion of the methodological approach). The vertical (horizontal) component of the Plumb flux attains maximum values in the lower (upper) troposphere (Plumb 1985). Thus, a succinct depiction of the three-dimensional energy propagation associated with quasi-stationary Rossby waves can be obtained by superposing contours of the vertical flux analyzed at a lower tropospheric level with horizontal flux vectors for an upper tropospheric level (e.g. Black 1997). To study the relationship between the wave field and the Plumb flux, it is also useful to overlay composite height anomalies (with the zonal mean removed) and the associated horizontal Plumb flux vectors. We employ both of these graphical approaches in figure 5.8 which displays Plumb flux analyses for the maturing stage of positive (upper panels) and 
negative (lower panels) NAM events (noting that in both cases the composite wave field is almost solely comprised of planetary scale waves - wavenumbers 1,2, and 3). For comparison, the climatological Plumb fluxes are displayed in the upper panels of figure 5.11 .

The left hand column of figure 5.8 displays the $300 \mathrm{mb}$ composite height anomalies (contours) along with the $300 \mathrm{mb}$ horizontal Plumb flux anomalies (vectors) while the right hand column overlays the same $300 \mathrm{mb}$ vector field with the $700 \mathrm{mb}$ vertical flux anomalies (contours). Before considering the Plumb flux results it is apparent that the composite height anomaly signatures are very different between the two NAM phases (figures 5.8a,c). Although both phases have pronounced height anomaly patterns over high latitudes of the north Atlantic, the corresponding oppositely-signed anomaly features have very different horizontal structures. The positive NAM events are characterized by a zonally elongated negative height anomaly centered just south of Greenland with positive height anomalies located to the east and south. In particular, we note the apparent southwest to northeast orientation of the latter anomaly feature, which for Rossby waves is associated with a southward (northward) eddy flux of wave activity (zonal momentum). This pattern is similar to the streamfunction anomaly pattern identified by DeWeaver and Nigam (2000, their figure 5a). For the negative NAM phase (figure 5.8c), the primary positive height anomaly feature over the north Atlantic is prominently distended from the northwest to the southeast which is normally associated with northward (southward) wave activity (zonal momentum) fluxes. Indeed, the corresponding Plumb flux vectors reveal prominent southeastward (northeastward) wave activity flux anomalies over the north Atlantic during maturing positive (negative) NAM 

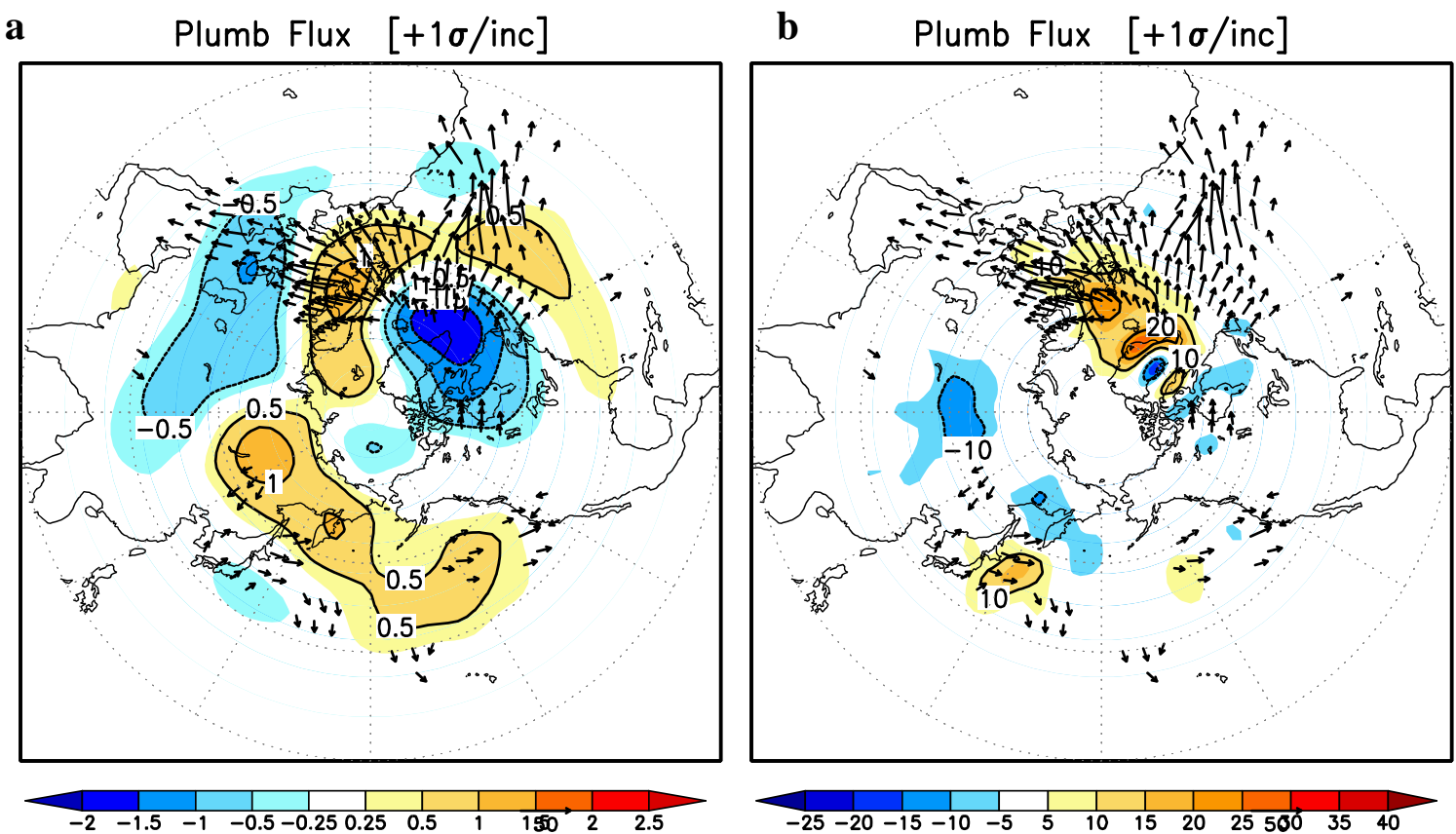

c
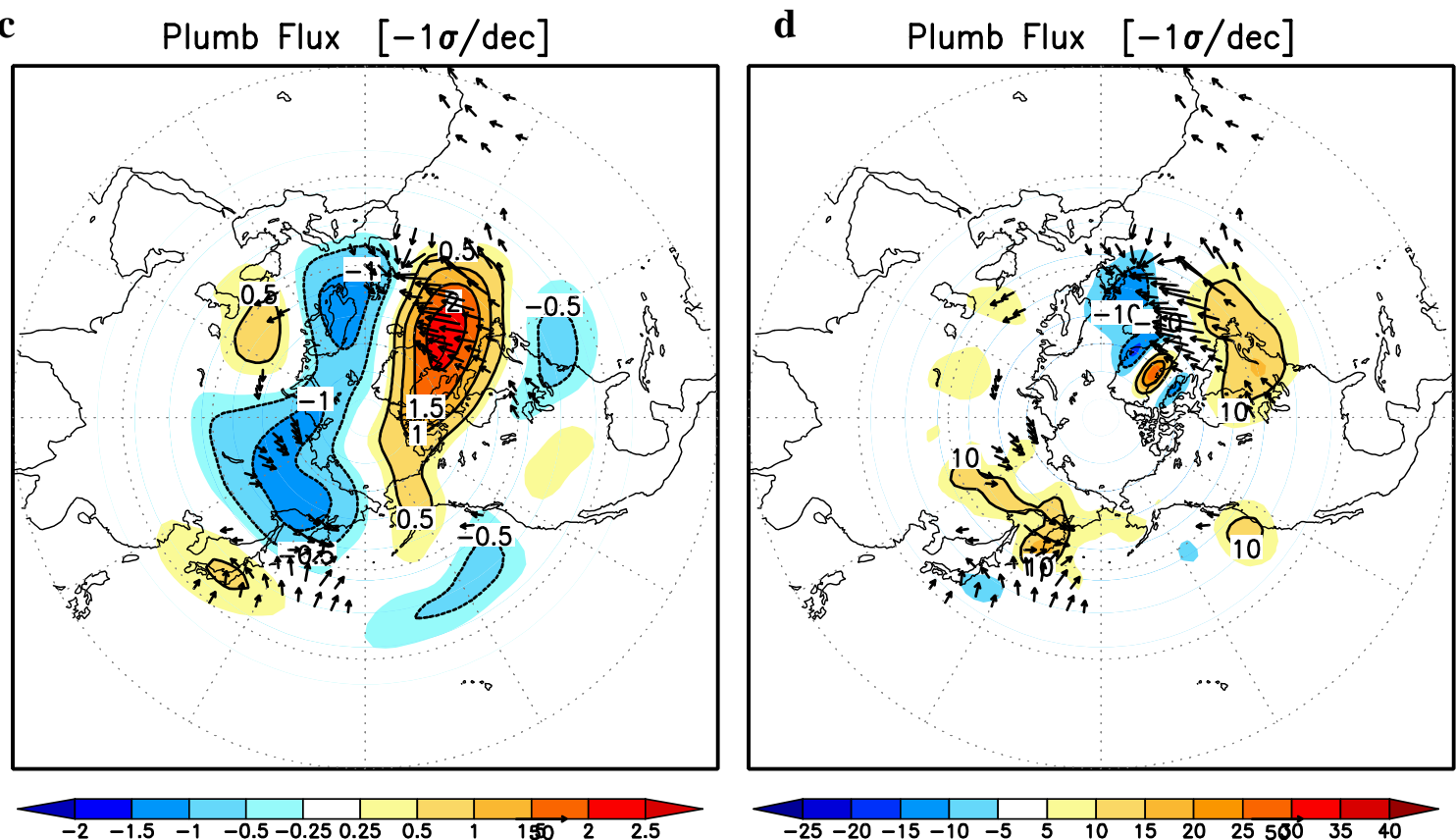

Figure 5.8 Three-dimensional Plumb flux anomalies for the positive (a) and (b) and negative (c) and (d) phases of the mature stage of the NAM due to the anomalous stationary waves. Figures (a) and (c) show the stationary height anomaly pattern (shaded, [units of $100 \mathrm{~m}$ ]) at $300 \mathrm{mb}$ and horizontal flux vectors at $300 \mathrm{mb}\left(\mathrm{m}^{2} / \mathrm{s}^{2}\right)$ while figures (b) and (d) show the horizontal flux vectors at $300 \mathrm{mb}$ and the vertical flux (contours/shading, units of $100 \mathrm{~m}^{2} / \mathrm{s}^{2}$ ) at $700 \mathrm{mb}$. 
events. This flux reversal parallels the monthly regression results of DeWeaver and Nigam (2000), but is occurring on a considerably shorter time scale.

The three-dimensional Plumb flux analyses provide further detail in indicating that positive NAM events are typified by a prominent upward and southeastward wave activity flux emanating from a local wave source located downstream of Greenland (figure 5.8b). This anomalous wave source is located northward of the regional wave source for the climatological stationary waves (e.g. Figure 4a of Plumb 1985), resulting in an effective northward shift in the total Plumb flux pattern (not shown). For negative NAM events there is an anomalous upward and northeastward wave flux pattern extending from a local wave source over the Canadian Maritimes to an apparent sink of wave activity (with downward fluxes) located east of Greenland (representing an effective southwestward shift in the total Plumb flux pattern). The identified wave source locations are consistent with local anomalies in orographic (positive NAM events) or thermal forcing (particularly negative NAM events). The latter possibility is discussed by Thompson and Wallace (2000) as possibly playing a role in forcing NAM events (see their Figure 11). The former possibility is consistent with the development of the horseshoe shaped zonal wind feature in figure $5.1 \mathrm{c}$, which would be associated with a reduced zonal flow anomaly over the orography of southern Greenland.

It is further revealing to note that parallel Plumb flux analyses for the two declining stages (not shown) show little or no coherent structure over the north Atlantic. This suggests that the decay of the NAM events may be linked to the disappearance of the local wave sources apparent in figure 5.8. In any case, it is clear that this composite study of regional wave dynamics reveals a strong lack of symmetry between positive and 
negative NAM events over the north Atlantic, in contrast to the zonal-mean dynamical analyses presented earlier. The net impact of these regional dynamical signatures upon the zonal mean circulation is explored in figures 5.9 and 5.10.

We next expand upon the EP-flux and wave driving analyses presented in figure 5.3 and 5.6 with temporal and spatial decompositions of the input eddy field (i.e. prior to calculating the eddy fluxes). The goal is to assess the relative roles of low frequency planetary waves and smaller scale synoptic eddies within the context of our zonal mean dynamical tendency analyses, noting that recent studies offer conflicting views of their respective roles in tropospheric NAM dynamics (e.g. DeWeaver and Nigam 2000, Benedict et al. 2004). In figures 5.9a and 5.9b we separately isolate the respective summed contributions of lowpass eddies and planetary-scale eddies toward the total EP flux and wave driving anomaly field for maturing positive NAM events. The good quantitative correspondence between figures 5.9a and 5.9b illustrates the approximate one-to-one relation between low frequency time scales and planetary spatial scales. A comparison with figure $5.3 \mathrm{~b}$ reveals that these large scale eddies comprise the majority of the total anomalous wave driving field in both the troposphere and stratosphere. The limited role of smaller scale eddies (wavenumbers 4 and higher, not shown) is to locally enhance the upper tropospheric wave driving maximum found near $60 \mathrm{~N}$.

The important point is that the quasi-stationary planetary scale waves appear to drive the daily zonal mean tendency dynamics, consistent with DeWeaver and Nigam's (2000) results for monthly time scales. An analysis of the zonal mean contribution of the Plumb flux anomalies to the tendency budget (figure 5.9c) further indicates that the composite anomaly field, itself composed almost solely of planetary waves (not shown), 

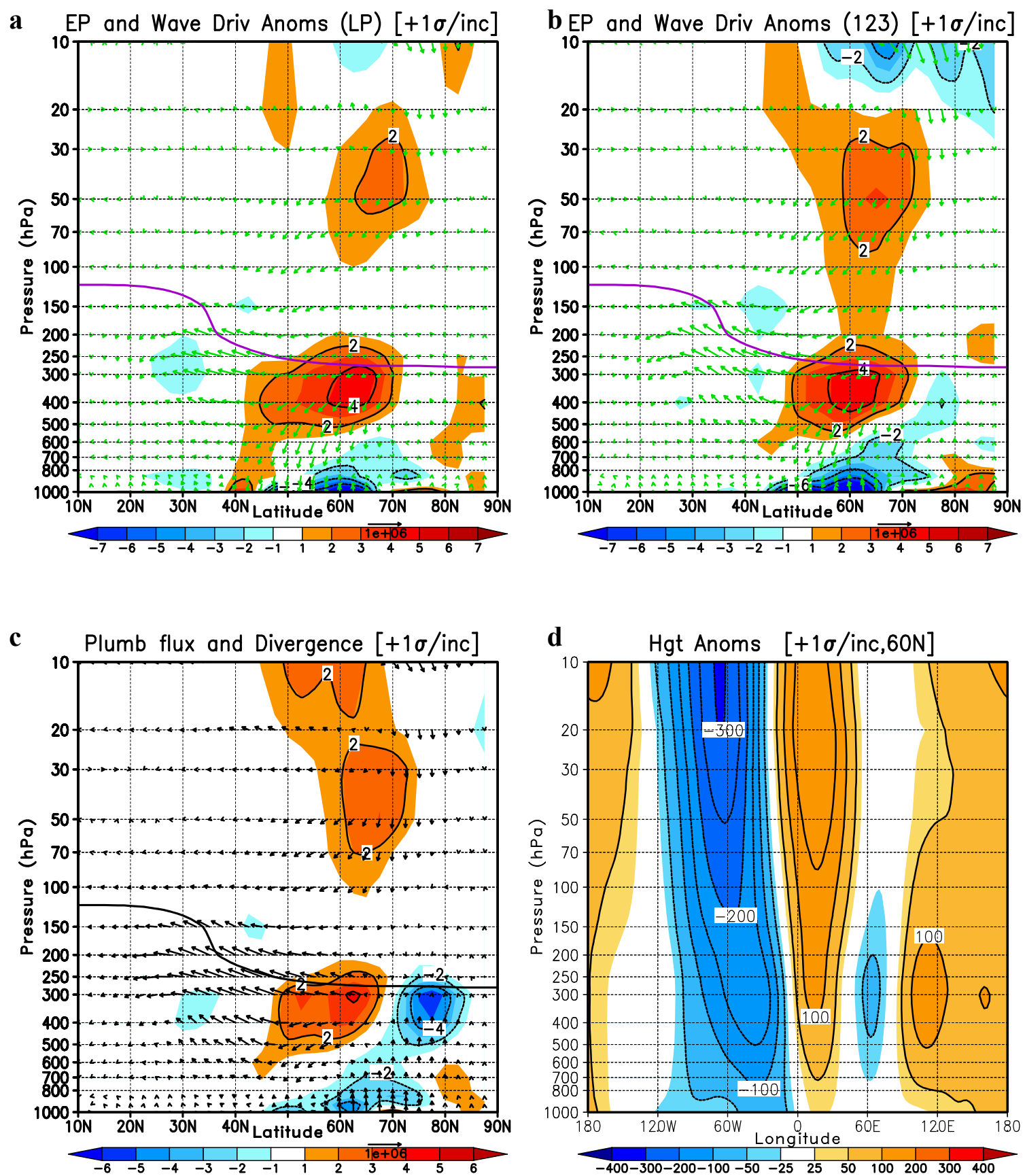

Figure 5.9 Latitude-pressure cross sections for the maturing stage of the positive NAM phase showing (a) anomalous EP-flux vectors $\left[\mathrm{m}^{3} \mathrm{~s}^{-2}\right]$ and associated wave driving $[\mathrm{m} / \mathrm{s} /$ day] due to LP eddies, (b) same as (a) but the forcing due to waves $1,2,3$, (c) a corresponding analysis using the meridional and vertical components of the zonalmean Plumb flux (derived from unfiltered composite anomalies), (d) longitudepressure cross section of the composite geopotential height anomalies at $60 \mathrm{~N}(50 \mathrm{~m}$ contours shown). 
accounts for most of the low frequency planetary scale forcing observed in figures 5.9a-b. This indicates that the composite anomaly field, by itself, likely contains the essential dynamical features critical to the tendency analyses, thereby providing important clues regarding regional forcing of the zonal mean NAM patterns. As such, and for completeness, we also display a longitude-pressure cross section of the composite wave anomalies at $60 \mathrm{~N}$ in figure $5.9 \mathrm{~d}$. We see clear evidence of coherent large-scale anomaly structures extending from the lower troposphere over the north Atlantic into the middle stratosphere. Comparing the anomaly field with the climatological stationary pattern shown in figure 5.11d, we see that, for the North Atlantic, the anomaly and climatological patterns are in phase, acting to enhance the trough west of Greenland.

Parallel diagnostic analyses for negative NAM events are presented in figure 5.10. We again find strong evidence that the total wave driving anomaly patterns (figure $5.6 \mathrm{~b}$ ) is largely due to low frequency, planetary scale eddies (figure 5.10a,b). Much of the anomalous wave driving field is explicitly contained in the composite wave field (figure 5.10c), itself characterized by deep quasi-stationary anomaly structures over the north Atlantic region. Unlike the positive phase, the stationary wave anomaly pattern (figure 5.10d) is out of phase with the climatological stationary wave pattern (5.11d), acting to decrease the North Atlantic trough. The important role of planetary scale waves in determining the tropospheric dynamics of negative NAM events contrasts with the results of Limpasuvan et al. (2004) who deduce a relatively more important role for wavenumbers 4 and higher in association with SSW events. We suggest that these differences are due to the different phenomenological foci of the two studies. Finally, we point out that the net zonal mean Plumb flux signatures evident in figures 5.9c and 5.10c 

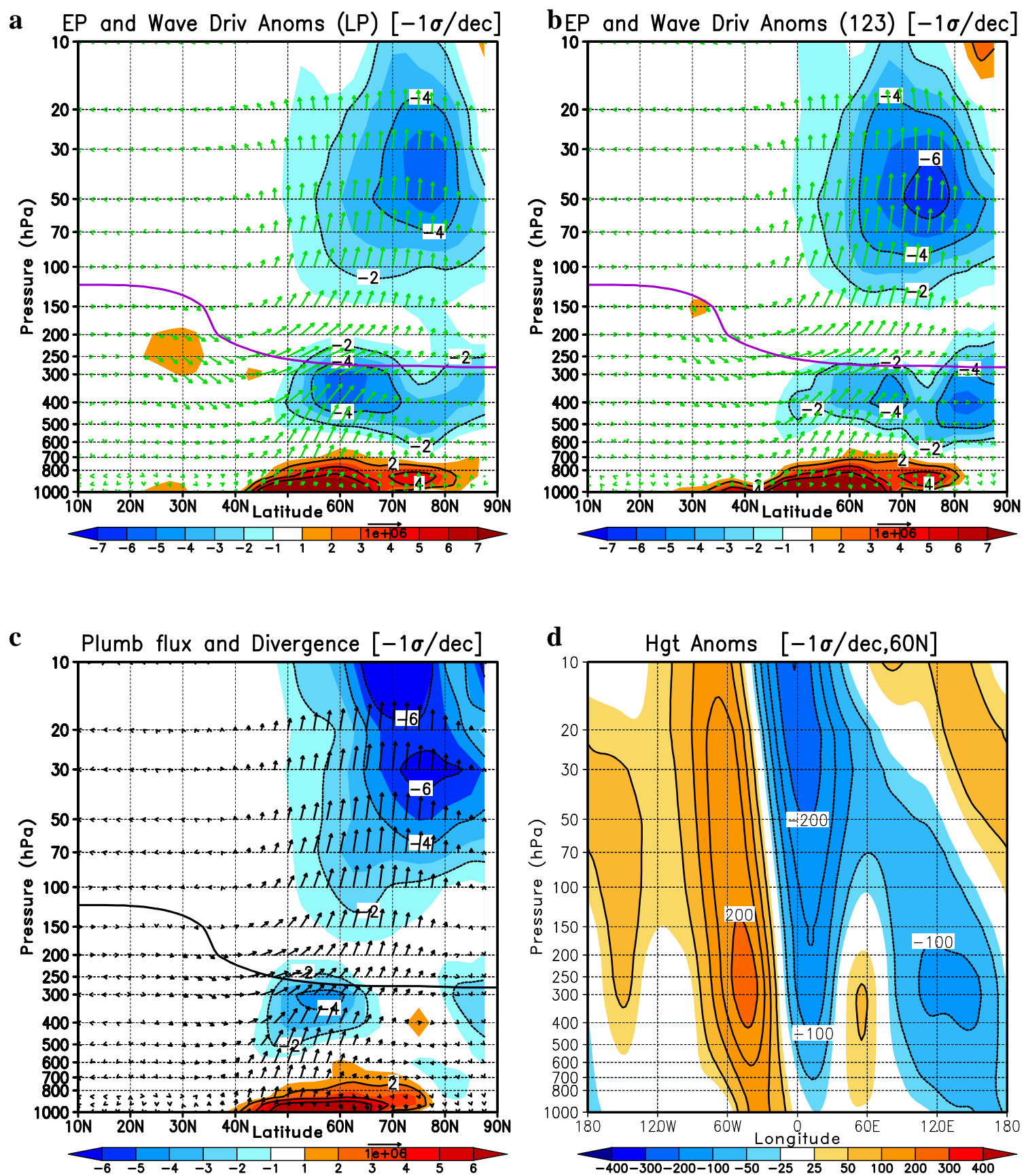

Figure 5.10 As in figure 5.9 but for the mature stage of the negative phase of the NAM. 
$\mathbf{a}$
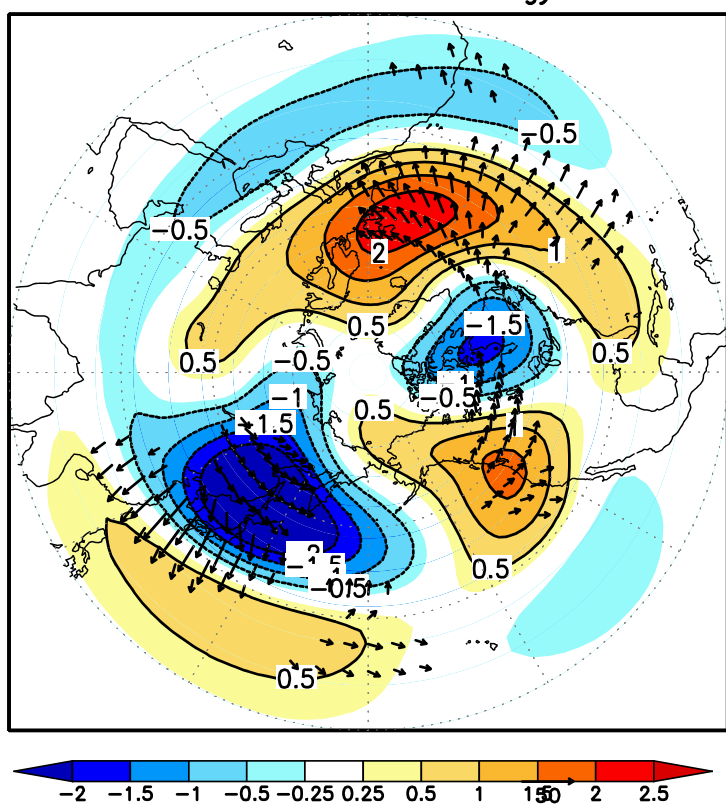

c

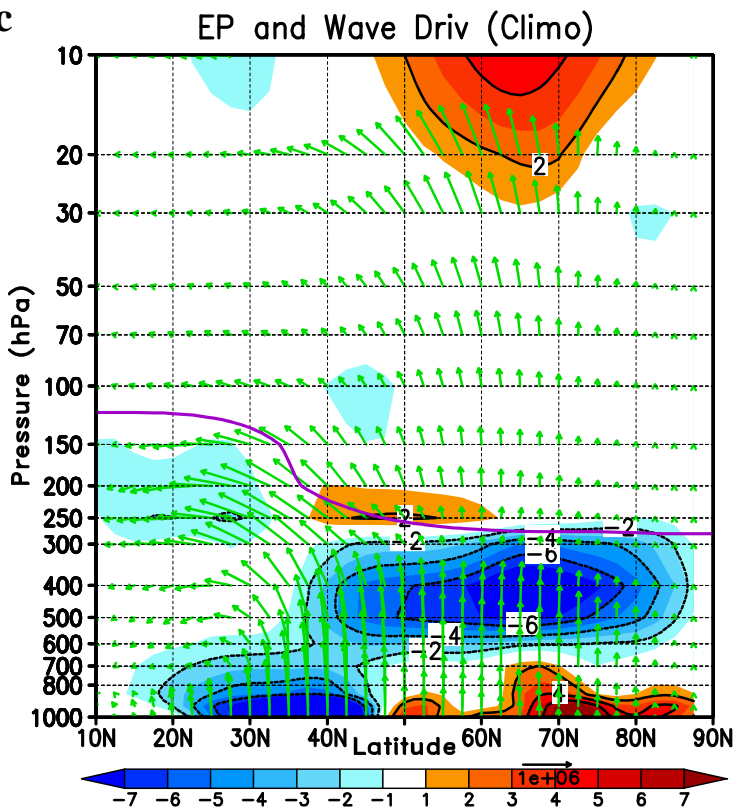

b Plumb Flux Climo (vert and hor)
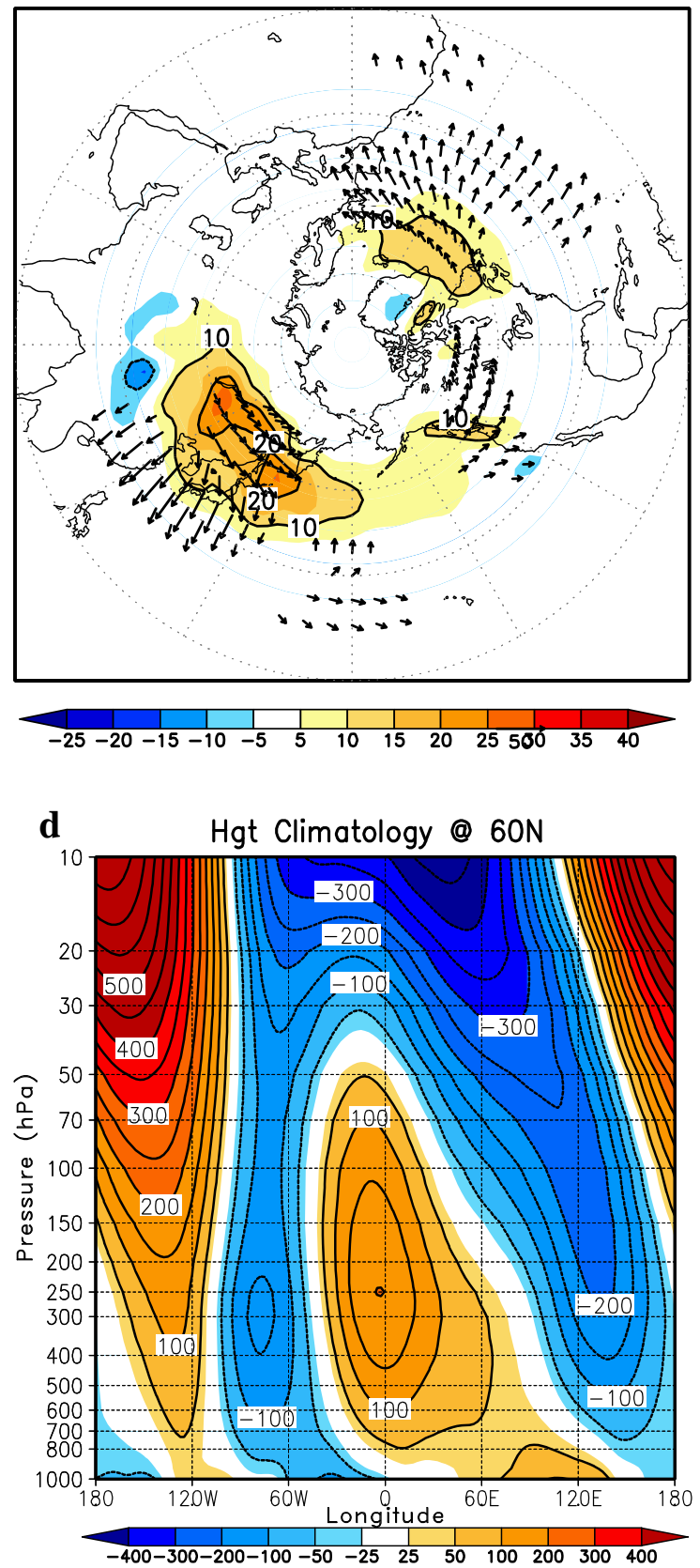

Figure 5.11 Climatological values of the Plumb flux (a) and (b) and the EP flux and associated wave driving (c). The climatological stationary wave pattern at $60 \mathrm{~N}$ is shown in panel (d). See figure 5.8 for details on panels (a) and (b), figure 5.3 for details on panel (c) and figure 5.9 for details on panel (d). 
effectively disappear between the maturing and declining stage of NAM events (consistent with the collapse of the regional Plumb flux pattern in figure 5.8). Therefore, unlike for the maturing stage, the anomalous wave driving pattens observed during NAM decay (figure $5.4 \mathrm{~b}$ and $5.7 \mathrm{~b}$ ) are primarily associated with case-to-case variability (the "incoherent" eddy field discussed in chapter 2). This may simply reflect the preferred manner in which the atmosphere responds when the regional forcing anomalies over the north Atlantic are removed.

\subsection{Summary}

The intraseasonal variability of the NAM has been studied by examining periods of strong NAM intensification/decline identified by studying the time rate of change of the NAM index. The growth and decay of NAM events each typically occur over a 1012 day period. The canonical NAM structure is evident in the composite zonal wind anomaly field for both the maturing and declining stages of NAM events. The wind tendency field undergoes a sign reversal during the transition from maturing to declining for both positive and negative phases of the NAM. A remarkable degree of reverse symmetry is revealed when examining the zonal-mean TEM analyses for corresponding stages of NAM development between the positive and negative phase of the NAM.

The EP flux and the associated driving, primarily forced by low-frequency, planetary scale eddies are largely responsible for forcing the daily zonal-wind tendencies in both the stratosphere and troposphere. The zonally-averaged Plumb flux indicates 
that, for the mature stage of the NAM, the composited anomaly fields themselves are able to account for most of the observed total anomalous wave driving. This indicates that the coherent eddies play a major role in the maturing stage of the NAM. From this we can conclude that most NAM events arise in a similar manner. In contrast, the declining stage does not exhibit significant coherent wave driving, leading to a conclusion that the breakdown of NAM events may occur in different fashions. Regional analyses of the height anomaly fields and the Plumb fluxes show a breakdown in the symmetry seen in the zonal mean fields. 


\section{CHAPTER 6}

\section{SUMMARY AND CONCLUSIONS}

\subsection{Summary}

The northern annular mode accounts for a significant fraction of the variance in the wintertime extratropical northern hemisphere and projects on timescales ranging from days to years (Thompson and Wallace 1998, Baldwin and Dunkerton 1999). Shorttimescale variations in the phase of the NAM impact regional weather patterns such as the likelihood of cold air outbreaks (Thompson and Wallace 2001). On longer timescales, interannual to decadal trends in the NAM index can alter regional climate characteristics with potentially serious implications upon the frequency of droughts and severe weather (e.g. Quadrelli et al 2001, Hurrell 1995).

Several studies have been performed that examined the intraseasonal dynamics inherent in the tropospheric and stratospheric manifestations of the NAM. However, these studies have been performed using monthly mean data and have concentrated on monthly-mean (or longer) dynamical balances. However, there have been very few diagnostic studies concentrating on the NAM dynamics for daily timescales. Limpasuvan et al (2004) examined the short-timescale dynamics of sudden stratospheric warmings but, as discussed in chapter 5, it is not evident that SSWs correspond well to the negative phase of the NAM. As a consequence, there remains considerable uncertainty concerning the mechanisms governing short timescale behavior of robust NAM events. We perform regression analyses, case studies, and composites based on 
periods of dynamical growth/decay to investigate the roles of the different proposed mechanisms in driving the atmospheric variability observed in association with the NAM on short intraseasonal timescales.

\section{(I) Regression Analyses}

We have performed regression analyses using the NAM index time series at 17 different pressure levels. This yields three-dimensional wind, pressure, and temperature anomaly fields as well as anomalous heat and momentum fluxes. From these analyses we obtain a detailed picture of the typical 21 day time evolution of the zonal wind and geopotential anomalies during intraseasonal NAM events. The EP-flux and wave driving fields were also analyzed in an attempt to determine the role of eddy forcing on the zonal mean flow variability.

The geopotential anomalies in the middle stratosphere at early stages of development are characterized by a robust negative anomaly feature centered over the pole and weaker, positive anomaly features over the midlatitude ocean basins. As the NAM signal descends, the magnitude of both the high- and mid- latitude geopotential features in the mid-stratosphere lessens and the geographic extent of the polar anomaly contracts. The positive anomaly feature in the North Atlantic all but vanishes midway through the 21-day development period while the Pacific feature decreases in magnitude and shifts eastward.

The tropospheric height anomaly patterns display considerably less annular symmetry, featuring more local maxima and minima. The strongest negative height anomalies, seen over the pole throughout the development period at stratospheric levels, shift toward the high-latitude North Atlantic. The largest positive height anomalies are 
centered over the eastern North Atlantic and western Europe, in contrast to the stratospheric levels where the largest positive anomalies are found over the Pacific basin.

The zonally-averaged zonal wind anomaly field displays deep, coherent westerlies (easterlies) at high (mid) latitudes at all stages of development. Even the early stages of development, when the signal is strongest in the mid and lower stratosphere, are characterized by substantial tropospheric wind anomalies. During latter stages the stratospheric wind anomalies weaken while the tropospheric features become more robust with an increasing meridional geographic extent near the surface.

During the early/middle stages of development, the high-latitude anomalous EP flux pattern and the associated wave driving show enhanced upward wave activity flux into the stratosphere and with anomalous EP flux convergence in the stratosphere. The convergence acts to slow the polar vortex, decreasing the stratospheric NAM signature as expected as the signal descends downward into the troposphere. This is comparable to the stratosphere in the declining stage of the positive phase of the NAM shown in the dynamical composites chapter. At later stages the vertical component of the EP-flux in the mid-troposphere changes sign, becoming negative and leading to an EP flux divergence in the upper troposphere. This acts to accelerate the zonal flow, helping to bring about the tropospheric manifestations of the NAM. At all stages of development the wave driving pattern is accompanied by an oppositely Coriolis torque, acting to maintain thermal wind balance during the disruption by the anomalous wave activity.

As shown in figure 3.7, linear regression analyses are not the best approach for examining short-timescale tendency dynamics. The regression operator obscures the time tendencies by equally sampling both periods of growth and decay and identifying 
anomaly structures for time periods when the NAM signal is at a maximum (and thus not experiencing significant zonal-wind tendencies). The regression analysis does provide a clear picture of the canonical zonal wind and height anomaly structures that characterize the NAM. Using the lag procedure we are also able to examine the descent of the NAM signal in these fields.

\section{(ii) Case Studies}

We have performed piecewise potential vorticity inversions and EP flux analyses of robust stratospheric NAM events with and without succeeding NAM manifestations. The PV inversion analyses of case 1 provide an event-oriented confirmation of the regression results of Black (2002) regarding the direct downward influence of stratospheric PV anomaly structures. The PV anomalies in the lower stratosphere act to induce a coherent annular pattern of westerly anomalies extending through the depth of the troposphere. The development of case 2 is also characterized by tropospheric zonal wind tendencies that are induced by stratospheric PV anomaly features. In this case, however, the stratospherically induced easterly acceleration is effectively offset by westerly wind anomalies associated with a preexisting positive tropospheric PV anomaly feature located near the pole. Thus, in this case, a direct downward influence is masked by a preexisting tropospheric annular mode feature. Therefore, even though the stratosphere clearly provides a significant forcing on the troposphere in case 2 , this is not ultimately manifested as a robust like-signed tropospheric NAM event.

Interestingly, case 3 is marked by the latitudinal shift of a preexisting coherent annular mode pattern that extends from the surface upward into the stratosphere but is initially out of phase with the canonical NAM anomaly pattern. The dynamics of this 
case are fundamentally different from cases 1 and 2 in that the tropospheric zonal wind changes that take place are almost solely driven by tropospheric PV anomaly tendencies. In this case, NAM onset in the troposphere (and lowermost stratosphere) is actually initiated directly by tropospheric rather than stratospheric PV dynamics. Of course, it remains possible that the stratosphere may have played an important role in exciting the initial, meridionally shifted annular mode structures. Although the behavior of case 3 is atypical among the cases studied here, the results nonetheless illustrate that tropospheric NAM events can be excited in different ways.

The inferences drawn using case 2 were more generally verified in separate composite analyses of the onset of several negative and positive nonpropagating events (NN and PN, respectively). The $\mathrm{NN}$ and $\mathrm{PN}$ composites are both characterized by preexisting and oppositely signed tropospheric circulation anomalies that conceal the role of the stratosphere in enacting subsequent tropospheric zonal anomalies. In contrast, the tropospheric zonal wind tendencies are nonzero. The EP flux diagnoses of both the case studies and composites indicate that the initial stratospheric circulation changes are induced by alterations in the propagation characteristics of tropospheric Rossby waves. The PN composite development is associated with anomalously weak upward fluxes of Rossby wave activity. This leads to a reduction in wave breaking (driving), resulting in a net acceleration of the zonal wind and northward fluxes of eddy potential vorticity. In contrast, the NN composites exhibit an enhanced upward flux of Rossby wave activity from the troposphere leading to enhanced stratospheric wave breaking (and southward fluxes of eddy PV), decelerating the zonal flow. Considering the PV inversion and EP fluxes jointly suggests that any direct downward influence on these timescale should 
likely be regarded as a feedback process having roots in the tropospheric circulations.

Our results indicate important roles for both direct and indirect forcing mechanisms in enacting stratospheric influences upon tropospheric climate. We conclude that whether or not a tropospheric NAM signal emerges from a given stratospheric NAM event is largely dependent on the detailed nature of any preexisting annular modes in the troposphere.

\section{(iii) Dynamical Composites}

We have studied the dynamical evolution of the northern annular mode using a composite analysis based on daily variations in the NAM index. Transformed Eulerian mean, potential vorticity, and regional Plumb flux diagnostics are employed to deduce proximate forcings of the zonal mean wind tendency during the growth and decay of large positive and negative NAM events. The respective role of eddies on different time scales and spatial scales are also determined.

The growth and decay of NAM events each typically occur over a 10-12 day period. A similar time scale is observed for the duration of large-amplitude NAM events. The canonical NAM structure is evident in the composite zonal wind anomaly field for both mature and decline stages of the NAM events. The main distinction is the appearance of a horseshoe-shaped anomaly structure in the troposphere during the decline (mature) stage of the positive (negative) NAM events. For both NAM phases the composite zonal wind tendency field undergoes a sign reversal during the transition between mature and decline stages.

The TEM analyses reveal a remarkable degree of reverse symmetry between positive and negative NAM events. During the mature stage, positive (negative) NAM 
events are associated with anomalous EP fluxes directed equatorward and downward (poleward and upward) inducing zonal wind accelerations (decelerations) within the upper troposphere and stratosphere at high latitudes. These patterns agree very well with the index of refraction ideas suggested by Hartmann et al. (2000). Specifically, when the polar vortex is anomalously strong (weak) it will act to deflect upwardly propagating tropospheric Rossby wave activity equatorward (poleward) leading to a net reduction (enhancement) in the amount of wave activity reaching the polar vortex as well as increase (decrease) the tropospheric wave driving. This tropospheric wave driving is also associated with robust anomalies in the tropospheric residual circulation which acts to vertically redistribute the zonal wind tendency response toward the lower troposphere. The transition from mature to decline stage is characterized by (I) a near collapse of the initially strong meridional EP flux feature centered near the midlatitude tropopause and (ii) a sign reversal in the vertical EP flux in both the troposphere and stratosphere. The latter feature is particularly evident in the mid-troposphere near 60N. Thus, NAM decay is not a simple radiative relaxation process but contains active dynamical forcing elements. Piecewise potential vorticity tendency inversion analyses indicate that for both positive and negative NAM events the direct tropospheric response to stratospheric PV tendencies is strongest during the mature stage, although still weak in magnitude compared to the proximate effects of wave driving.

Regional three-dimensional analyses of the composite circulation observed during the mature stage of NAM events reveal prominent planetary scale anomaly features over the North Atlantic that extend upward well into the stratosphere. There is a lack of symmetry between positive and negative NAM events, however. For positive (negative) 
events the primary tropospheric circulation anomaly is distended zonally (northwest to southeast) and is characterized by anomalous southeastward (northeastward) wave activity fluxes extending from a local wave source located east of Greenland (over the Canadian Maritimes). We speculate that these anomalous wave sources are likely related to variations in the thermal and/or orographic forcing of the quasi-stationary wave field. Interestingly, for both positive and negative NAM events these anomalous wave source over the North Atlantic virtually disappear between the mature and decline stages. The lack of a distinct anomalous Plumb flux and height anomaly pattern may indicate that, while the mature composites are dominated by the coherent eddy fluxes and thus little case to case variability, the incoherent eddies play a significant role in the decay stage. This implies that different NAM events (both positive and negative) reach the mature stage in distinct, consistent ways but decay in a less consistent manner. This suggests that NAM decay may be linked to the cessation of localized anomalous wave forcing over the North Atlantic region (One hypothetical example might be the termination of anomalous cold air advection in association with the depletion of an upstream cold air mass).

The dynamical nature of the wave driving was explored by temporal and spatial decompositions of the input eddy fields. These analyses indicate that the anomalous wave driving signatures in both the stratosphere and troposphere are mainly due to low frequency (intraseasonal periods greater than 10 days) planetary scale (wavenumbers 1,2, and 3) eddies. The role of smaller scale (wavenumbers 4 and higher) eddies is to provide secondary enhancement to the upper tropospheric wave driving patterns near $60 \mathrm{~N}$. Further, zonal-mean analyses of the regional wave activity flux field indicate that 
the composite anomaly field, itself, is able to account for most of the low frequency planetary scale wave driving. This suggests that the composite anomaly field contains the fundamental essence of the intraseasonal onset dynamics of the NAM.

Similar to past studies we find that stratospheric variations in the NAM are strongly driven by variations in upwardly propagating tropospheric planetary scale waves (e.g., Hartmann et al. 2000, Limpasuvan et al. 2004). We also provide strong diagnostic evidence supporting the idea that short term intraseasonal variations in the tropospheric NAM are also primarily driven by low frequency planetary scale waves. Although this result appears at odds with the synoptic analysis of Benedict et al. (2004), it is entirely consistent with the thesis put forth by DeWeaver and Nigam (2000) regarding the monthly forcing of the zonal mean flow by the dynamical interplay between anomalous and climatological stationary waves over the North Atlantic. Our study suggests that this forcing mechanism likely acts on submonthly timescales as well. Although we anticipated better correspondence between the results of Limpasuvan et al. (2004) and our composite analyses of negative NAM events, we found important distinctions in both the composite anomaly structures and in the dynamical signatures. The primary distinction is the relative role of low frequency planetary scale eddies in determining the tropospheric NAM dynamics. We suggest that these differences arise from a lack of correspondence (particularly in the troposphere) between the SSW events studied by Limpasuvan et al. (2004) and the negative NAM events studied here.

We conclude that low frequency planetary scale waves provide the primary dynamical forcing for short term intraseasonal variations in the NAM in both the stratosphere and troposphere. More specifically, we find strong evidence that this forcing 
is due to large-scale quasi-stationary anomalies over the North Atlantic region. Further, we have determined that NAM decay is linked to a collapse in the pattern of anomalous stationary wave forcing over the North Atlantic, which in turn helps promote the observed changes in the wave driving of the zonal mean flow. Although our diagnostic study provides evidence for both direct and indirect downward stratospheric influences

during NAM events, the former appears relatively weak in comparison to the latter. The current results provide an important observational benchmark for future theoretical and modeling studies of intraseasonal NAM dynamics.

\subsection{Theoretical Implications}

Using the results of this study, we can evaluate the role of earlier posited mechanisms of intraseasonal NAM variability. This is important as some of the proposed mechanisms may not apply to short term variability (and vice versa). To summarize, the proposed mechanisms are:

(a) Vertical reflection of upwardly propagating Rossby waves [originating within the troposphere] within the stratosphere (Perlwitz and Harnik 2003).

(b) Direct forcing of the tropospheric circulation by stratospheric potential vorticity (PV) anomalies (Ambaum and Hoskins 2002, Black 2002, Black and McDaniel 2004, McDaniel and Black 2005).

C) Variations in the lower stratospheric zonal wind alter the upward propagation of tropospheric Rossby waves. This mechanism has both a direct component (anomalous tropospheric wave driving) and a secondary component (reduction/enhancement of the 
polar vortex by altered tropospheric wave propagation into the stratosphere that can lead to a positive feedback). Studies have differed on the role of synoptic and planetary scale eddies in this mechanism (e.g. Hartmann et al. 2000, Limpasuvan et al. 2004, McDaniel and Black 2005, Lorenz and Hartmann 2003).

Our approach is unsuited to explicitly assess the Rossby wave reflection mechanism. Therefore we cannot rule out this mechanism. We do find substantial evidence for the direct downward forcing of the tropospheric circulation by stratospheric PV anomalies. Large stratospheric PV anomalies are shown to induce robust anomalous tropospheric responses with significant anomalies present near the surface. However, this mechanism is not always evident as shown in the third case study where tropospheric PV anomaly structures are responsible for much of the observed anomalous circulation.

Our results provide considerable clarification of the eddy forcing mechanism and the role of different time/spatial scale eddies. During the maturing (declining) stage of the positive phase of the NAM, upwardly propagating tropospheric waves are ducted equatorward (upward). This leads to acceleration (deceleration) in both the midlatitude troposphere and the stratospheric polar vortex. [This pattern is reversed for the maturing and declining stages of the negative phase of the NAM.]. For both the positive and negative phases of the NAM, the tropospheric momentum fluxes nearly vanish during the transition from maturing to declining while the heat fluxes in both the troposphere and stratosphere change sign. The zonally averaged Plumb flux, when compared to the EP flux, illustrates that the quasi-stationary, planetary scale waves provide the majority of anomalous wave driving signature during the maturing stage. The correspondence between the zonally averaged plumb flux and the EP flux is not present during the 
declining stages. This illustrates that, while the wave driving in the maturing composites is primarily due to the coherent eddies (thus indicating that events tend to arise in the same manner), the declining stages tend to occur in different manners. For both maturing and declining stages, we find little contribution from the synoptic scale eddies. This may indicate that, while the canonical wind structures for the negative phase of the NAM and the SSWs may have similarities, different dynamical processes are occurring in the two phenomena.

A useful summary is to provide a theoretical, mechanistic evolution of a standard negative NAM event:

Anomalous tropospheric momentum and heat fluxes (centered over the North Atlantic), associated with quasi-stationary planetary waves, give rise to an anomalous upward and poleward tropospheric wave activity flux, part of which acts to slow the upper tropospheric zonal flow. There is also an enhanced upward tropospheric wave activity flux into the stratosphere. These waves then break within the polar vortex, acting to mechanically slow the vortex. The stratospheric wave breaking leads to a local thermal wind imbalance. The adjustment to thermal wind imbalance produces a north-south dipole in the stratospheric PV anomaly field that is associated with anomalous easterlies in both the stratosphere and troposphere. The new stratospheric and tropospheric zonal wind anomalies may act to alter the propagation of tropospheric Rossby waves, a potential positive feedback, allowing for persistence in the phase of the NAM. This pattern persists until a change occurs in the troposphere over the North Atlantic, altering the tropospheric heat flux and effectively ceasing the momentum flux. 


\subsection{The Future}

A better understanding of the dynamics inherent to stratosphere-troposphere interactions is needed in order to improve numerical weather forecasting at all time scales. As a subset of this larger problem, deducing the mechanisms occurring during NAM events may lead to increased forecast skill in the extratropics. Predicting robust stratospheric NAM events may improve the forecast ability for severe weather events at or near the surface. Understanding and predicting long-term trends in the variability associated with the NAM may improve regional climate forecasts on monthly to annual timescales and allow policy makers and industries to better prepare for regional climate shifts.

What scientific advances are required to better understand and predict the NAM? First, the role of Rossby wave reflection in NAM dynamics needs to be assessed in a more comprehensive manner. Second, a more complete understanding of the regional wave forcing of the NAM using both observations and model output is essential. Equally important is deducing the proximate mechanistic sources of anomalies in the regional stationary wave forcing, delineating between the roles of thermal and orographic forcing.

To fully identify the proximate planetary wave sources, analyses must be performed using a three-dimensional numerical model. This will allow the direct attribution of mountain torque and thermal forcing to the overall wave field. However, we can speculate on the North Atlantic wave sources using the results of the dynamical composites. For the positive phase of the NAM, orographic forcing may be the primary wave source. Enhanced zonal flow over the topography of southern Greenland (during 
the maturing stage) can lead to enhanced upwardly propagating Rossby waves.

Examining the Plumb fluxes shown in figure 5.8b, we observe vertical wave activity above and downstream of Greenland, consistent with the proposed mechanism. During the declining stage of the positive phase, the zonal wind anomalies present over Greenland are removed, thus eliminating the wave source.

During the negative phase of the NAM, the enhanced zonal flow over Greenland is not present and thus the above mechanism is not applicable. The role of thermal forcing must therefore be examined. Figure 6.1 displays the difference in temperature anomalies from maturing to declining stages for both the positive and negative phases of the NAM. A zonally oriented temperature dipole is evident over North America and the western North Atlantic during the negative phase with a large negative temperature anomaly centered over Canada and a positive anomaly located over the ocean. This shows that the normal temperature gradient present over this region (cold land, warmer ocean) is enhanced. This can lead to anomalous activity in the thermally forced wave field. The location of the temperature dipole corresponds well with the location of vertical Plumb flux (figure 5.8d). A simplified mechanism for this pattern is as follows: negative wind anomalies at $60 \mathrm{~N}$ (figure 5.5a) signify less than normal zonal flow over the (cold) North American continent. This leads to a lack of westerly cold air advection, meaning the land will remain cold while the lack of cold air advection implies that the downstream ocean will be anomalously warm. This enhances the climatological cold land-warm ocean pattern, potentially leading to increased Rossby wave propagation. Eventually the negative zonal wind anomalies diminish, meaning the pool of cold air over the continent is advected (as normal) downstream. This restores the climatological 

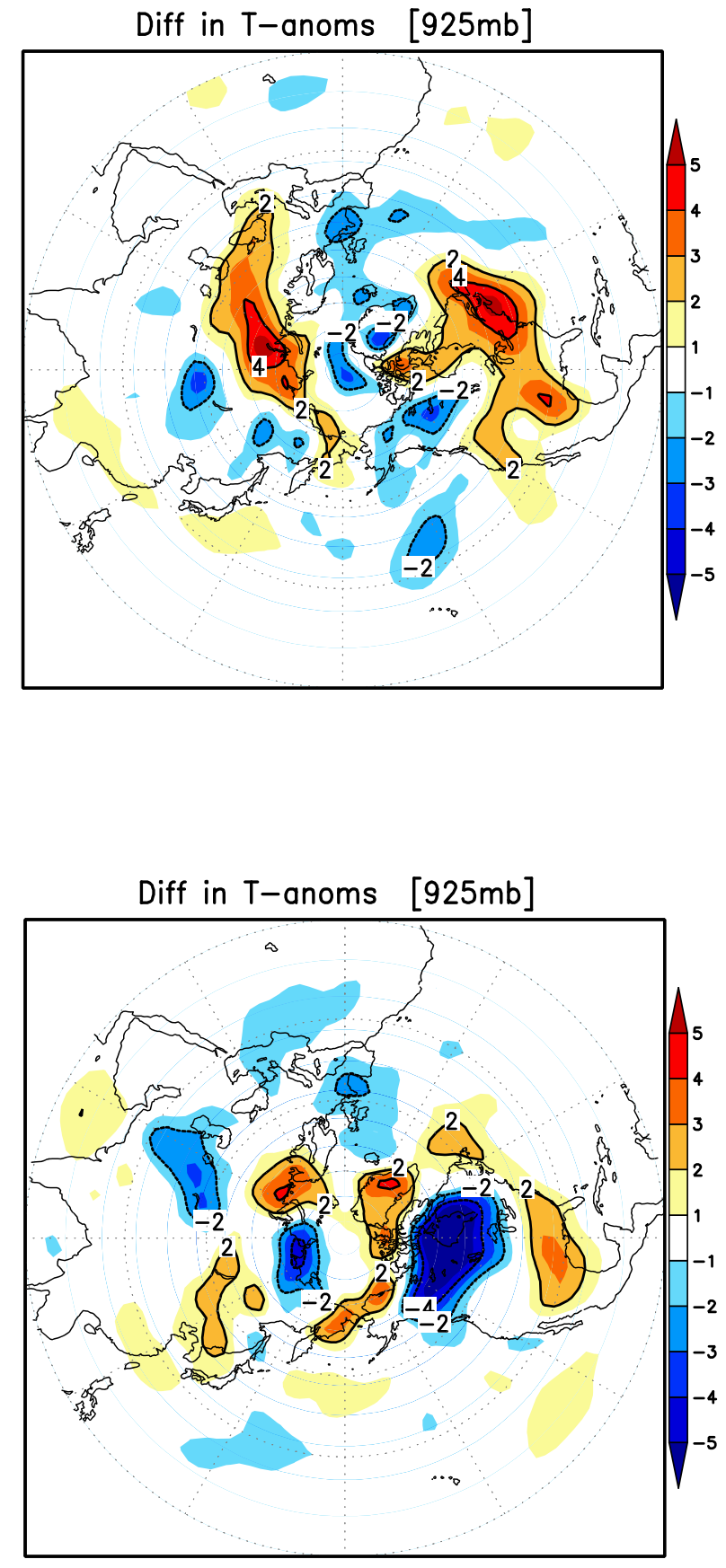

Figure 6.1 Polar stereographic plots showing the difference in the temperature anomaly field between the maturing and declining stages for (a) the positive phase and (b) the negative phase of the NAM. 
temperature gradient, thus removing the anomalous wave source.

The role of tropospheric synoptic scale eddies, while not determined to be a primary source of significant wave driving in this study, needs also to be clarified in NAM dynamics. A better resolved stratosphere in large climate and numerical weather prediction models, while already beginning to be implemented, will also likely improve our mechanistic understanding. 


\section{References}

Ambaum, M. H. P., B. J. Hoskins, and D. B. Stephenson, 2001: Arctic Oscillation or North Atlantic Oscillation? J. Climate, 14, 3495-3507.

Ambaum, M.H.P., and B.J. Hoskins, 2002: The NAO troposphere-stratosphere connection. J. Climate, 15, 1969-1978.

Andrews, D. G., J. R. Holton, and C. B. Leovy, 1987: Middle Atmosphere Dynamics. Academic Press, 489 pp.

Baldwin, M. P., and T. J. Dunkerton, 1999: Propagation of the Arctic Oscillation from the stratosphere to the troposphere. J. Geophys. Res., 104, 30937-30946.

Baldwin, M.P., and T. J. Dunkerton, 2001: Stratospheric Harbingers of Anomalous Weather Regimes. Science, 294, 581-584.

Baldwin, M. P., H. J. Edmon, and J. R. Holton, 1985: A diagnostic study of eddy-mean flow interactions during FGGE SOP-1. J. Atmos. Sci., 43, 1838-1845.

Benedict, J. J., S. Lee, and S. B. Feldstein, 2004: Synoptic view of the North Atlantic Oscillation. J. Atmos. Sci., 61, 121-144.

Black, R. X., 1997: Deducing anomalous wave source regions during the life cycles of persistent flow anomalies. J. Atmos. Sci., 54, 895-907.

Black, R. X., 1998: The maintenance of extratropical intraseasonal transient eddy activity in the GEOS-1 assimilated dataset. J. Atmos Sci., 55, 3159-3175.

Black, R. X., 2002: Stratospheric forcing of surface climate in the Arctic oscillation. $J$. Climate, 15, 268-277..

Black, R.X. and B. A. McDaniel, 2004: Diagnostic Case Studies of the Northern Annular Mode. J. Climate, 17, 3990-4004.

Chen, P., and W. A. Robinson, 1992: Propagation of planetary waves between the troposphere and stratosphere. J. Atmos. Sci., 49, 2533-2545.

Davis, C. A., 1992: Piecewise potential vorticity inversion. J. Atmos. Sci., 49, 13971411.

DeWeaver, E., and S. Nigam, 2000: Do stationary waves drive the zonal-mean jet anomalies of the Northern Winter? J. Climate, 13, 2160-2176.

DeWeaver, E., and S. Nigam, 2000: Zonal-eddy dynamics of the North Atlantic 
Oscillation. J. Climate, 13, 3893-3914.

Duchon, C.E., 1979: Lanczos filtering in one and two dimensions. J. Applied Met., 18, 1016-1022.

Edmon, H. J., B. J. Hoskins, and M. E. McIntyre, 1980: Eliassen-Palm cross sections for the troposphere. J. Atmos. Sci., 37, 2600-2616.

Fyfe, J.C., G.J. Boer, and G.M. Flato, 1999: The Arctic and Antarctic Oscillations and their Projected Changes Under Global Warming. Geophys. Res. Lett, 26, 16011604.

Grotzner, A., M. Latif, T. P. Barnett, 1998: A decadal climate cycle in the North Atlantic Ocean as simulated by the ECHO coupled GCM. J. Climate, 11, 831-847.

Hartley, D.E., J. Villarin, R.X. Black, and C.A. Davis, 1998: A new perspective on the dynamical link between the stratosphere and troposphere. Nature, 391, 471-474.

Hartmann, D. L., J. M. Wallace, V. Limpasuvan, D. W. J. Thompson, and J. R. Holton, 2000: Can ozone depletion and global warming interact to produce rapid climate change? P Natl. Acad. Sci. USA., 97, 1412-1417.

Hastenrath, S., and L. Greischar. 2001: The North Atlantic oscillation in the NCEP-NCAR reanalysis. J. Climate, 14, 2404-2413.

Haynes, Peter, 2005: Stratospheric Dynamics. Annual Rev. Fluid Mechanics, 37, $263-$ 293.

Haynes, P.H., C. J. Marks, M. E. McIntyre, T.G. Shepherd, and K. P. Shine, 1991: On the 'downward control' of extratropical diabatic circulations by eddy-induced mean zonal forces. J. Atmos. Sci., 48, 651- 678.

Hoskins, B. J., M. E. McIntyre, and A. W. Robertson, 1985: On the use and significance of isentropic potential vorticity maps. Quart. J. Roy. Meteor. Soc., 111, 877-946.

Hoskins, B.J. and D.J. Karoly, 1981: Steady linear response of a spherical atmosphere to thermal and orographic forcing. J. Atmos. Sci., 38, 1179-1196.

Hurrell, J. W., 1995: Decadal trends in the North Atlantic Oscillation region temperatures and precipitation. Science, 269, 676-679.

Kalnay, E., and Coauthors, 1996: The NCEP/NCAR 40-year reanalysis project. Bull. Amer. Meteor. Soc., 77, 437-471.

Lewis, R. P. W., Ed., 1991: Meteorological Glossary. $6^{\text {th }}$ ed. Her Majesty's Stationer's Office, $335 \mathrm{pp}$. 
Limpasuvan, V., and D.L. Hartmann, 1999: Eddies and the Annular Modes of Climate Variability. Geophys. Res. Lett., 26, 3133-3136.

Limpasuvan, V., D. W. J. Thompson, and D. L. Hartmann, 2004: On the life cycle of Northern Hemisphere stratospheric sudden warmings. J. Clim., 17, 2584-2596.

Lorenz, E. N., 1951: Seasonal and irregular variations of the northern hemisphere sealevel pressure profile. J. Meteorol., 8, 52-59.

Lorenz, D.J. and D.L. Hartmann, 2001: Eddy-Zonal Flow Feedback in the Southern Hemisphere. J. Atmos. Sci., 58, 3312-3327.

Lorenz, D.J. and D.L. Hartmann, 2003: Eddy-zonal flow feedback in the Northern Hemisphere winter. J. Climate, 16, 1212-1227.

McDaniel, Brent A. and R.X. Black, 2005: Intraseasonal Dynamical Evolution of the Northern Annular Mode. J. Climate, (in press).

Namias, J., 1950: The index cycle and its role in the general circulation. J. Meteorol., 7, 130-139.

O’Neill, A. and B. F. Taylor, 1979: A study of the major stratospheric warming of 1976/77. Quart. J. Roy. Meteor. Soc., 105, 71-92.

Palmer, T. N., 1981: Properties of the Eliassen-Palm flux for planetary scale motions. $J$. Atmos. Sci., 39, 992-997.

Perlwitz, J. and N. Harnik, 2003: Observational evidence of a stratospheric influence on the troposphere by planetary wave reflection. J. Climate, 16, 3011-3026.

Plumb, R. Alan, 1985: On the three-dimensional propagation of stationary waves. $J$. Atmos. Sci., 42, 217-229.

Polvani, L. M., and P. J. Kushner, 2002: Tropospheric response to stratospheric perturbations in a relatively simple general circulation model. Geophys. Res. Lett., 29, No. 7, doi:10.1029/2001GL014284.

Polvani, L. M., and D. W. Waugh, 2004: Upward Wave Activity Flux as Precursor to Extreme Stratospheric Events and Subsequent Anomalous Surface Weather Regimes. J. Climate, 17, 3548-3554.

Press, William H., Saul A. Teukolsky, William T. Vetterling, and Brian P. Flannery, 1992: Numerical Recipes in Fortran. University of Cambridge Press, 959 pp.

Quadrelli, R., V. Pavan, and F. Molteni, 2001: Wintertime variability of Mediterranean 
precipitation and its links with large-scale circulation anomalies. Clim. Dynam., 17, 457-466.

Reichler, Thomas, P. J. Kushner, and L. Polvani, 2005: The coupled stratospheretroposphere response to impulsive forcing from the troposphere. J. Atmos. Sci.. In Press.

Robinson, W. A., 2000: A Baroclinic Mechanism for the Eddy Feedback on the Zonal Index. J. Atmos. Sci., 57, 415-422.

Rodwell, M. J., D. P. Rowell, and C. K. Folland, 1999: Oceanic forcing of the wintertime North Atlantic Oscillation and European climate. Nature, 398, 320-323.

Rossby, C., 1939: Relations between variations in the intensity of the zonal circulation of the atmosphere and the displacement of the semipermanent centers of action. $J$. Mar. Res., 3, 38-55.

Rossby, C. and H.C. Willett, 1948: The circulation of the upper troposphere and lower stratosphere. Science, 108, 643-652.

Shepherd, Theodore G., 2002: Issues in Stratosphere-troposphere Coupling. J. Met. Soc. Japan, 80, 769-792.

Shindell, D. T., R. L. Miller, G. A. Schmidt, and L. Pandolfo, 1999: Simulation of recent northern winter climate trends by greenhouse-gas forcing. Nature, 399, 452-455.

Solomon S, 1999: Stratospheric ozone depletion: A review of concepts and history. Rev. Geophys., 37, 275-316.

Song, Y. and W.A. Robinson: Dynamical mechanisms for stratospheric influences on the troposphere. J. Atmos Sci., 61, 1711-1725.

Thompson, D. W. J., and J. M. Wallace, 1998: The Arctic Oscillation signature in the wintertime geopotential height and temperature fields. Geophys. Res. Lett., 25, 1297-1300.

Thompson, D. W. J., and J. M. Wallace, 2000: Annular modes in the extratropical circulation. Part I: Month-to-month variability. J. Climate, 13, 1000-1016.

Thompson, D. W. J., and J. M. Wallace, 2001: Regional Climate Impacts of the Northern Hemisphere Annular Mode. Science, 293, 85-89.

Thompson, D. W. J., S. Lee, and M.P. Baldwin, 2002: Atmospheric Processes Governing the Northern Hemisphere Annular Mode/North Atlantic Oscillation. From the AGU monograph on the NAO. J. W. Hurrell, Y. Kushnir, M. Visbeck, G. 
Ottersen, Eds.

van Loon, H. and J. Rogers, 1978: The seesaw in winter temperatures between Greenland and Northern Europe. Part I: General Description. Mon. Wea. Rev., 106, 296310.

Walker, G. T., and E. W. Bliss, 1932: World Weather V. Mem. Roy. Meteor. Soc., 4, No. $36,53-84$.

Wallace, J. M., 2000: North Atlantic Oscillation/Northern Hemisphere annular mode, 2000: One phenomenon, two paradigms. Q. J. Roy. Met. Soc., 126, 791-805.

Willett, H.C., 1948: Patterns of world weather change. Trans. Amer. Geophys. Union. 29, 803-809.

Wittman, M. A. H., Polvani, L.M., Scott, R.K., Charlton, A.J.,2004. Stratospheric influence on baroclinic lifecycles and its connection to the Arctic Oscillation. Geo. Rev. Letters, 31, L16113, doi:10.1029/2004GL020503.

Zeng, Guang and John A. Pyle, 2003: Changes in tropospheric ozone between 2000 and 2100 modeled in a chemistry-climate model. Geophys. Res. Lett., 30, art. no. 1392.

Zhou, S. A. J. Miller, J. Wang, and J. K. Angell, 2002: Downward propagating temperature anomalies in the preconditioned polar stratosphere. J. Climate, 15, 781-792. 